\title{
COHERENT CHOICE FUNCTIONS, DESIRABILITY AND INDIFFERENCE
}

\author{
ARTHUR VAN CAMP, GERT DE COOMAN, ENRIQUE MIRANDA, AND ERIK QUAEGHEBEUR
}

\begin{abstract}
We investigate how to model indifference with choice functions. We take the coherence axioms for choice functions proposed by Seidenfeld, Schervisch and Kadane as a source of inspiration, but modify them to strengthen the connection with desirability. We discuss the properties of choice functions that are coherent under our modified set of axioms and the connection with desirability. Once this is in place, we present an axiomatisation of indifference in terms of desirability. On this we build our definition of indifference in terms of choice functions, which we discuss in some detail.
\end{abstract}

\section{INTRODUCTION}

The fundamental problem of study in decision theory is how to make a choice from within a set of available options. Rubin [21] proposed a theory of choice functions based on choices between more than two elements, where an agent determines which are the optimal ones for any input set. He started from von Neumann and Morgenstern's [33] axiomatisation of choices based on pairwise comparisons between the options.

One aspect of Rubin's axiomatisation is that the agent is assumed to be able to make a comparison between any pair of options (either one of them is strictly preferred to the other or they are indifferent). As a consequence, he is assumed to be indifferent between any two options chosen from a given set. However, the agent may be undecided between two options without being indifferent between them, particularly when the information available does not allow for a complete comparison of the options; this will for instance typically be the case when there is no relevant information available at all. This is one of the motivations for a theory of imprecise probabilities [34], where incomparability and indifference are distinguished. This point was also discussed by Kadane et al. [16] (and also [27]), who generalised Rubin's axioms [21] in order to allow for incomparability. One interesting aspect of their take on choice functions is that it does not require any predefined utility.

Imprecise probability is an umbrella term for mathematical models that are meant to be used in situations of imprecise or incomplete information, where it may not be possible (or advisable) to use (precise) probabilities. In particular, it covers sets of probability measures and various types of non-additive measures and functionals, such as coherent lower previsions, belief functions and possibility measures. All of these models can be expressed in terms of coherent sets of desirable gambles [19, 34, 35], which encode the gambles that a subject, whose beliefs we want to model, strictly prefers to the status quo. One of their advantages is that they avoid problems with conditioning on events of probability zero. They can be-and have been-used to replace probabilities in Bayesian networks, for predictive inference, and so on [5, 7, 11, 13, 18].

Sets of desirable gambles are typically a binary concept: they are characterised by pairwise comparisons between the available options, whereas in practice choice may be more complex. Choice functions based on binary comparisons only may represent behaviour that is considered to be paradoxical by some [3, 14, 22]. One of the aims of this paper is to 
study how the more general — not necessarily binary-choice functions relate to the sets of desirable gambles that are now more commonly used in imprecise probabilities papers.

In order to establish such a relationship, we must pay some attention at this point to the options that subjects choose between on both approaches: gambles on the desirability approach, and horse lotteries in a choice function context. A detailed discussion of this issue can be found in a recent paper by Zaffalon and Miranda [36], but for our present purposes it suffices to mention that choosing between gambles essentially amounts to choosing between horse lotteries with only two rewards - say, winning a prize and not winning it - and the value of a gamble is essentially proportional to the probability of winning the prize on the corresponding horse lottery approach. This correspondence makes it relatively straightforward to translate the axioms proposed by Seidenfeld et al. [27] for choice functions on horse lotteries into their counterparts for gambles: we prefer to work with gambles here because that makes it easier to see the link with sets of desirable gambles. We will find it expedient to drop two particular axioms from the theory proposed by Seidenfeld et al. [27], thus making our coherence notion less restrictive. The first one is the Archimedean axiom, which is not fully compatible with the theory of coherent sets of desirable gambles - indeed it is letting go of Archimedean continuity that allows coherent sets of desirable gambles to avoid the problems that arise when conditioning on sets of probability zero. We have also not included the convexity axiom of Seidenfeld et al. [27], since it is incompatible with Walley-Sen maximality [30, 34], the optimality criterion typically associated with sets of desirable gambles. We discuss the axioms for our coherent choice functions and their main properties in Section 2 .

In Section 3, we show that models of coherent sets of desirable gambles can be embedded within our theory of coherent choice functions, and we study the specific properties of choice functions that can be related to desirability. This exploratory work culminates in Section 4, where we focus on sets of probability measures and collections of maximal coherent sets of desirable gambles.

Once the connection between choice functions and desirability is in place, we move-in Section 5-to a definition and closer investigation of the property of indifference between two options. This is a crucial step towards enabling uncertainty models to represent symmetry [9]. For example, when modelling exchangeability and proving de Finetti-type representation theorems, an appropriate notion of indifference is essential. For choice functions, indifference was introduced by Seidenfeld [24], and for sets of desirable gambles by de Cooman and Quaeghebeur [11].

The connection between choice functions and sets of desirable gambles established in Section 3 leads us to investigate how to model choice that is compatible with a set of indifference assessments. Our approach to defining indifference focuses on the quotient space induced by the indifference relation. This allows us to exploit notions and techniques from linear algebra to deal with it mathematically. It also enables us to prove a representation result that makes it easy to consider conservative inference under indifference. We show how our notion is related to the one established by Seidenfeld [24].

We conclude the paper with some additional discussion in Section 6 . In order not to impede the main flow of the argumentation, we have moved all proofs to an appendix.

\section{CHOICE FUNCTIONS ON OPTION SETS}

Consider a real vector space $\mathcal{V}$, provided with the vector addition + and scalar multiplication. We denote by 0 the additive identity, or null vector. For any subsets $O_{1}$ and $O_{2}$ of $\mathcal{V}$ and any $\lambda$ in $\mathbb{R}$, we define $\lambda O_{1}:=\left\{\lambda u: u \in O_{1}\right\}$ and $O_{1}+O_{2}:=\left\{u+v: u \in O_{1}, v \in O_{2}\right\}$. 
Elements $u$ of $\mathcal{V}$ are intended as abstract representations of options amongst which a subject can express his preferences, by specifying, as we will see below, choice functions. Mostly, options will be real-valued maps on the possibility space, interpreted as uncertain rewards, and therefore also called gambles. Our reason for working with the more abstract notion of options-elements of some general vector space-is that in Section 5, we will need choice functions defined on equivalence classes of options. These equivalence classes again constitute a vector space-and hence are abstract options themselves-but they can no longer be interpreted easily and directly as gambles. Another reason for using options more general than gambles is that recent work [36] has shown that a very general theory of binary preference can be constructed using vector-valued gambles. These are no longer gambles in the above-mentioned sense, but still constitute a linear space.

We denote by $\mathcal{Q}(\mathcal{V})$ the set of all non-empty finite subsets of $\mathcal{V}$, a strict subset of the power set of $\mathcal{V}$. Elements $O$ of $\mathcal{Q}(\mathcal{V})$ are the option sets amongst which a subject can choose his preferred options. When it is clear what vector space of options we are talking about, we will omit explicit mention of $\mathcal{V}$ and simply write $\mathcal{Q}$.

Definition 1. A choice function $C$ on $\mathcal{Q}$ is a map

$$
C: \mathcal{Q} \rightarrow \mathcal{Q} \cup\{\varnothing\}: O \mapsto C(O) \text { such that } C(O) \subseteq O .
$$

We collect all choice functions in the set $\mathcal{C}$.

The idea underlying this definition is that a choice function $C$ selects the set $C(O)$ of 'best' options in the option set $O$, or, on another interpretation, the ones that cannot be rejected. Our definition resembles the one commonly used in the literature [1, 27, 29], except for a restriction to finite option sets, which, then again, is also not altogether unusual [15, 23, 28].

2.1. An equivalent representation. An equivalent notion of a choice function is that of a binary relation-called choice relation-on $\mathcal{Q}$.

Definition 2. A choice relation $\triangleleft$ is a binary relation on $\mathcal{Q}$ that satisfies the following two properties for all $O, O_{1}$ and $O_{2}$ in $\mathcal{Q}$ :

(i) if $O_{2} \backslash O_{1} \subseteq O \subseteq O_{1} \cup O_{2}$ then $O_{1} \triangleleft O_{2} \Leftrightarrow O_{1} \triangleleft O$;

(ii) if $O_{1} \cup O_{2} \subseteq O$ then $\left(O_{1} \triangleleft O\right.$ and $\left.O_{2} \triangleleft O\right) \Leftrightarrow O_{1} \cup O_{2} \triangleleft O$.

We collect all choice relations in the set $\mathcal{S}$.

Given a choice function, we can use it to define a choice relation, and vice versa:

Definition 3. Given a choice function $C$, define the corresponding binary relation $\triangleleft_{C}$ as

$$
O_{1} \triangleleft_{C} O_{2} \Leftrightarrow C\left(O_{1} \cup O_{2}\right) \subseteq O_{2} \backslash O_{1} \Leftrightarrow C\left(O_{1} \cup O_{2}\right) \cap O_{1}=\varnothing \text { for all } O_{1} \text { and } O_{2} \text { in } \mathcal{Q} \text {. }
$$

Conversely, given a choice relation $\triangleleft$, define the corresponding function $C_{\triangleleft}$ as

$$
C_{\triangleleft}(O):=\bigcap\left\{O^{\prime} \subseteq O: O \backslash O^{\prime} \triangleleft O\right\}=O \backslash \bigcup\left\{O^{\prime} \subseteq O: O^{\prime} \triangleleft O\right\} \text { for all } O \text { in } \mathcal{Q} .
$$

The interpretation is clear: we find that $O_{1} \triangleleft_{C} O_{2}$ if $u \notin C\left(O_{1} \cup O_{2}\right)$ for all $u$ in $O_{1}$-in other words, when every option from $O_{1}$ is not chosen-rejected-when presented with $O_{1} \cup O_{2}$ as option set. Furthermore, for $C_{\triangleleft}$, an option $u$ is rejected from $O$-meaning that $u \in O$ and $u \notin C_{\triangleleft}(O)$-if there is some $O^{\prime} \subseteq O$ for which $u \in O^{\prime}$ and $O^{\prime} \triangleleft O$-in other words, if there is some $O^{\prime}$ that contains $u$ and such that $O$ is preferred to $O^{\prime}$.

Starting from a choice relation $\triangleleft$, it is clear from Definition 3 that $C_{\triangleleft}$ is a choice function-meaning that $C_{\triangleleft}(O) \subseteq O$ for all $O$ in $\mathcal{Q}$. We prove the converse argument-that $\triangleleft_{C}$ satisfies the two properties of Definition 2 - in the following proposition: 
Proposition 1. Given a choice function $C$, the corresponding binary relation $\triangleleft_{C}$ is a choice relation.

Interestingly, we can use $C$ and $\triangleleft$ interchangeably:

Proposition 2. Given any choice function $C$, we have $C_{\triangleleft_{C}}=C$. Similarly, given any choice relation $\triangleleft$, we have $\triangleleft_{C_{\triangleleft}}=\triangleleft$. As a consequence, $\triangleleft_{C}=\triangleleft \Leftrightarrow C_{\triangleleft}=C$ for all $C$ in $\mathcal{C}$ and $\triangleleft$ in $\mathcal{S}$.

2.2. Rationality axioms. Seidenfeld et al. [27], Section 3] call a choice function $C$ coherent if there is a non-empty set of probability-utility pairs $\mathcal{S}$ such that $C(O)$ is the set of options in $O$ that maximise expected utility for some element of $\mathcal{S}$. They also provide an axiomatisation for this type of coherence, based on the one for binary preferences [2]. One of their axioms is an 'Archimedean' continuity condition, and another one is a convexity condition, necessary for the connection with a set of probability-utility pairs.

We prefer to define coherence directly in terms of axioms, without reference to probabilities and utilities. In such a context, we see no compelling reason to adopt an Archimedean axiom, all the more so because we are interested in establishing the connection between choice functions and Walley]' [35] sets of desirable gambles, which need not satisfy this axiom. Furthermore, the convexity condition does not generally allow for choice functions that select the undominated options under some partial ordering, which is something we find natural, and will need later on.

For these reasons, we propose an alternative—and in some sense weaker-axiomatisation in Section 2.2.2 by dropping the Archimedean condition and the convexity condition of Seidenfeld et al. [27]. Then again, our second axiom is a slightly strengthened version of theirs, needed for the conditioning we intend to discuss in a later paper.

2.2.1. Some useful definitions. We call $\mathbb{N}$ the set of all (strictly positive) integers, and $\mathbb{N}_{0}:=\mathbb{N} \cup\{0\}$. Also, we call $\mathbb{R}_{>0}$ the set of all (strictly) positive real numbers, and $\mathbb{R}_{\geq 0}:=$ $\mathbb{R}_{>0} \cup\{0\}$.

Given any subset $O$ of $\mathcal{V}$, we define the linear hull $\operatorname{span}(O)$ as the set of all finite linear combinations of elements of $O$ :

$$
\operatorname{span}(O):=\left\{\sum_{k=1}^{n} \lambda_{k} u_{k}: n \in \mathbb{N}, \lambda_{k} \in \mathbb{R}, u_{k} \in O\right\} \subseteq \mathcal{V},
$$

the positive hull posi $(O)$ as the set of all positive finite linear combinations of elements of $O$ :

$$
\operatorname{posi}(O):=\left\{\sum_{k=1}^{n} \lambda_{k} u_{k}: n \in \mathbb{N}, \lambda_{k} \in \mathbb{R}_{>0}, u_{k} \in O\right\} \subseteq \operatorname{span}(O),
$$

and the convex hull $\mathrm{CH}(O)$ as the set of convex combinations of elements of $O$ :

$$
\mathrm{CH}(O):=\left\{\sum_{k=1}^{n} \alpha_{k} u_{k}: n \in \mathbb{N}, \alpha_{k} \in \mathbb{R}_{\geq 0}, \sum_{k=1}^{n} \alpha_{k}=1, u_{k} \in O\right\} \subseteq \operatorname{posi}(O) .
$$

A subset $O$ of $\mathcal{V}$ is called a convex cone if it is closed under positive finite linear combinations, i.e. if $\operatorname{posi}(O)=O$. A convex cone $\mathcal{K}$ is called proper if $\mathcal{K} \cap-\mathcal{K}=\{0\}$.

With any proper convex cone $\mathcal{K} \subseteq \mathcal{V}$, we associate an ordering $\leq \mathcal{K}$ on $\mathcal{V}$, defined for all $u$ and $v$ in $\mathcal{V}$ as follows:

$$
u \leq \mathcal{K} v \Leftrightarrow v-u \in \mathcal{K} \Leftrightarrow 0 \leq_{\mathcal{K}} v-u \Leftrightarrow u-v \leq_{\mathcal{K}} 0 .
$$

We also write $u \geq_{\mathcal{K}} v$ for $v \leq_{\mathcal{K}} u$. The ordering $\leq_{\mathcal{K}}$ is actually a vector ordering: it is a partial order-reflexive, antisymmetric and transitive — that satisfies the following two 
characteristic properties:

$$
\begin{aligned}
& u_{1} \leq_{\mathcal{K}} u_{2} \Leftrightarrow u_{1}+v \leq_{\mathcal{K}} u_{2}+v ; \\
& u_{1} \leq_{\mathcal{K}} u_{2} \Leftrightarrow \lambda u_{1} \leq_{\mathcal{K}} \lambda u_{2},
\end{aligned}
$$

for all $u_{1}, u_{2}, v$ in $\mathcal{V}$ and $\lambda$ in $\mathbb{R}_{>0}$. Conversely, given any vector ordering $\leq$, the proper convex cone $\mathcal{K}$ from which it is derived can always be retrieved by $\mathcal{K}=\{u \in \mathcal{V}: u \geq 0\}$. When the abstract options are gambles, $\leq$ will typically be the pointwise order. When the options are equivalence classes, as in Section 5.2 the ordering will be typically the induced ordering on equivalence classes, defined in Equation (13) further on.

The vector space of options $\mathcal{V}$, ordered by the vector ordering $\leq_{\mathcal{K}}$, is called an ordered vector space $\langle\mathcal{V}, \leq \mathcal{K}\rangle$. We will refrain from explicitly mentioning the actual proper convex cone $\mathcal{K}$ we are using, and simply write $\mathcal{V}$ to mean the ordered vector space, and use $\leq$ for the associated vector ordering.

Finally, with any vector ordering $\leq$, we associate the strict partial ordering $<$ as follows:

$$
u<v \Leftrightarrow(u \leq v \text { and } u \neq v) \Leftrightarrow v-u \in \mathcal{K} \backslash\{0\} \text { for all } u, v \text { in } \mathcal{V} .
$$

We call $u$ positive if $u>0$, and collect all positive options in the convex cone $\mathcal{V}_{>0}:=\mathcal{K} \backslash\{0\}$.

At this point, it is important to mention that from here on, we assume the cone $\mathcal{K}$ to be fixed and given. It serves to identify a 'positive orthant' $\mathcal{V}_{>0}=\mathcal{K} \backslash\{0\}$, and should not be confused with the sets of desirable options (defined further on in Section 3.1), which are cones as well, and typically include the positive orthant $\mathcal{V}_{>0}$.

\subsubsection{Rationality axioms for choice functions.}

Definition 4. We call a choice function $C$ on $\mathcal{Q}(\mathcal{V})$ coherent if for all $O, O_{1}, O_{2}$ in $\mathcal{Q}$, all $u, v$ in $\mathcal{V}$ and all $\lambda$ in $\mathbb{R}_{>0}$ :

$\mathrm{C}_{1} . C(O) \neq \varnothing$;

$\mathrm{C}_{2}$. if $u<v$ then $\{v\}=C(\{u, v\})$;

$\mathrm{C}_{3}$. a. if $C\left(O_{2}\right) \subseteq O_{2} \backslash O_{1}$ and $O_{1} \subseteq O_{2} \subseteq O$ then $C(O) \subseteq O \backslash O_{1}$;

b. if $C\left(O_{2}\right) \subseteq O_{1}$ and $O \subseteq O_{2} \backslash O_{1}$ then $C\left(O_{2} \backslash O\right) \subseteq O_{1}$;

$\mathrm{C}_{4}$. a. if $O_{1} \subseteq C\left(O_{2}\right)$ then $\lambda O_{1} \subseteq C\left(\lambda O_{2}\right)$;

b. if $O_{1} \subseteq C\left(O_{2}\right)$ then $O_{1}+\{u\} \subseteq C\left(O_{2}+\{u\}\right)$.

We collect all coherent choice functions on $\mathcal{V}$ in the set $\overline{\mathcal{C}}$.

Remark that Axiom $\mathrm{C}_{3} \mathrm{~b}$ is well defined, in the sense that none of $C$ 's arguments there can be empty. Indeed, $O_{2} \backslash O$ is guaranteed to be non-empty: since $O \subseteq O_{2} \backslash O_{1}$, we find that $O_{2} \backslash O$ must include the set $O_{1}$, which is non-empty by Axiom $\mathrm{C}_{1}$ into account.

Parts $\mathrm{C}_{3} \mathrm{a}$ and $\mathrm{C}_{3} \mathrm{~b}$ of Axiom $\mathrm{C}_{3}$ are respectively known as Sen's condition $\alpha$ and Aizerman's condition. They are more commonly written as, respectively:

$$
\left(O_{1} \cap C\left(O_{2}\right)=\varnothing \text { and } O_{1} \subseteq O_{2} \subseteq O\right) \Rightarrow O_{1} \cap C(O)=\varnothing \text { for all } O, O_{1}, O_{2} \text { in } \mathcal{Q},
$$

and

$$
\left(O_{1} \cap C\left(O_{2}\right)=\varnothing \text { and } O \subseteq O_{1}\right) \Rightarrow O_{1} \cap C\left(O_{2} \backslash O\right)=\varnothing \text { for all } O, O_{1}, O_{2} \text { in } \mathcal{Q} .
$$

The first one means that any option that is rejected in a option set $\mathrm{O}_{2}$ must also be rejected in any larger option set $O$ (i.e., the inclusion of new options in the set cannot make an option that was rejected to become acceptable), while the second one means that if we reject an option in $\mathrm{O}_{2}$ we should also reject it when we remove some options that are also rejected from the option set (i.e., the removal of rejected options does not alter the judgments about the remaining ones). 
The following proposition collects a number of immediate properties of coherent choice functions.

Proposition 3. The following statements hold for any coherent choice function $C$ :

(i) $\lambda C(O)+\{u\}=C(\lambda O+\{u\})$ for all $O$ in $\mathcal{Q}, \lambda$ in $\mathbb{R}_{>0}$ and $u$ in $\mathcal{V}$;

(ii) for all $u_{1}, u_{2}$ in $\mathcal{V}$ such that $u_{1} \leq u_{2}$, all $O$ in $\mathcal{Q}$ and all $v$ in $O \backslash\left\{u_{1}, u_{2}\right\}$ :

a. if $u_{2} \in O$ and $v \notin C\left(O \cup\left\{u_{1}\right\}\right)$ then $v \notin C(O)$;

b. if $u_{1} \in O$ and $v \notin C(O)$ then $v \notin C\left(\left\{u_{2}\right\} \cup O \backslash\left\{u_{1}\right\}\right)$;

(iii) $C$ is insensitive to the omission of non-chosen options [12, Definition 11]: $C\left(O^{\prime}\right)=$ $C(O)$ for all $O, O^{\prime}$ in $\mathcal{Q}$ such that $C(O) \subseteq O^{\prime} \subseteq O$;

(iv) $C(C(O))=C(O)$ for all $O$ in $\mathcal{Q}$.

As already announced and motivated earlier, our axioms in Definition 4 are a subset of the ones considered by Seidenfeld et al. [27], who use horse lotteries as the basic options a subject must choose between. The set of all horse lotteries plays a similar role there as our vector space of options $\mathcal{V}$ does here. We omit two of Seidenfeld et al.'s [27] axioms: the Archimedean axiom, ensuring some continuity of coherent choice functions, and a convexity axiom. However, in further work we do intend to study the implications of adding the convexity axiom in the context of the relation between choice functions and sets of desirable options.

Our Axiom $\mathrm{C}_{2}$ is a slightly strengthened version of one of Seidenfeld et al.] [27] axioms. To compensate for this slightly weaker requirement, they impose the two properties (ii)a and (ii)b of our Proposition 3 as separate rationality axioms [27, Axiom 4]. Our proofs for them rely quite heavily on, amongst other things, our slightly stronger Axiom $C_{2}$. Our reason for using this strengthened version is that we want to concentrate on the relationship with desirability: any choice function that does not satisfy Axiom $\mathrm{C}_{2}$ does not induce a coherent set of desirable gambles, as we will see in Section 3.3 .

Seidenfeld et al.'s [27] call a choice function $C$, defined on the set $\mathcal{Q}(\mathcal{H})$ of all finite subsets of the set $\mathcal{H}$ of all horse lotteries, coherent if

$\mathrm{C}_{1}^{*} . C(O) \neq \varnothing$ for all $O$ in $\mathcal{Q}(\mathcal{H})$;

$\mathrm{C}_{2}^{*}$. for all $O$ in $\mathcal{Q}(\mathcal{H})$ and $H, H_{1}$ and $H_{2}$ in $\mathcal{H}$ such that $H \notin\left\{H_{1}, H_{2}\right\}$ and $H_{1} \leq H_{2}$ :

a. if $H_{2} \in O$ and $H \notin C\left(\left\{H_{1}\right\} \cup O\right)$ then $H \notin C(O)$;

b. if $H_{1} \in O$ and $H \notin C(O)$ then $H \notin C\left(\left\{H_{2}\right\} \cup O \backslash\left\{H_{1}\right\}\right)$;

$\mathrm{C}_{3}^{*}$. for all $O, O_{1}$ and $O_{2}$ in $\mathcal{Q}(\mathcal{H})$ :

a. if $C\left(O_{2}\right) \subseteq O_{2} \backslash O_{1}$ and $O_{1} \subseteq O_{2} \subseteq O$ then $C(O) \subseteq O \backslash O_{1}$;

b. if $C\left(O_{2}\right) \subseteq O_{1}$ and $O \subseteq O_{2} \backslash O_{1}$ then $C\left(O_{2} \backslash O\right) \subseteq O_{1}$;

$\mathrm{C}_{4}^{*} \cdot O_{1} \triangleleft_{C} O_{2} \Leftrightarrow\left(\alpha O_{1}+(1-\alpha)\{H\}\right) \triangleleft_{C}\left(\alpha O_{2}+(1-\alpha)\{H\}\right)$, for all $O_{1}$ and $O_{2}$ in $\mathcal{Q}(\mathcal{H})$ and $H$ in $\mathcal{H}$;

$\mathrm{C}_{5}^{*}$. if $O \subseteq O_{1} \subseteq \mathrm{CH}(O)$ then $C(O) \subseteq C\left(O_{1}\right)$, for all $O$ and $O_{1}$ in $\mathcal{Q}(\mathcal{H})$;

$\mathrm{C}_{6}^{*}$. for all $O, O^{\prime}, O^{\prime \prime}$ in $\mathcal{Q}(\mathcal{H})$ and all sequences $O_{i}{ }^{\prime}$ and $O_{i}{ }^{\prime \prime}$ in $\mathcal{Q}(\mathcal{H})$ such that $O_{i}{ }^{\prime}$ converges point-wise to $O^{\prime}$ and $O_{i}{ }^{\prime \prime}$ converges point-wise to $O^{\prime \prime}$ (for $i$ in $\mathbb{N}$ ):

a. if $(\forall i \in \mathbb{N}) O_{i}{ }^{\prime \prime} \triangleleft_{C} O_{i}{ }^{\prime}$ and $O^{\prime} \triangleleft_{C} O$ then $O^{\prime \prime} \triangleleft_{C} O$;

b. if $(\forall i \in \mathbb{N}) O_{i}{ }^{\prime \prime} \triangleleft_{C} O_{i}{ }^{\prime}$ and $O \triangleleft_{C} O^{\prime \prime}$ then $O \triangleleft_{C} O^{\prime \prime}$;

where $\leq$ is some predetermined reflexive order on $\mathcal{H}$.

Seidenfeld et al.'s [27] choice functions are defined on horse lotteries while we define our choice functions on any linear space, so direct comparison is not immediate. But there is a very close formal resemblance between the two sets of axioms. Indeed, Axioms $\mathrm{C}_{1}$ and $\mathrm{C}_{1}^{*}$ correspond; so do Axioms $\mathrm{C}_{3}$ and $\mathrm{C}_{3}^{*}$, and-perhaps less obviously-Axioms $\mathrm{C}_{4}$ and $\mathrm{C}_{4}^{*}$ Axiom $\mathrm{C}_{2}^{*}$, being a dominance condition, is essentially Proposition 3 (ii), and can be regarded as a weaker counterpart of Axiom $\mathrm{C}_{2}$. In the present setting, we are not interested 
in Axiom $\mathrm{C}_{5}^{*}$ because it is in general not compatible with maximality as a decision rule; neither are we interested in Axiom $\mathrm{C}_{6}^{\star}$ because it is a continuity requirement that is not fully compatible with sets of desirable gambles.

2.2.3. Rationality axioms for choice relations. It turns out we can formulate the rationality axioms for choice functions-Definition 4 -directly in terms of choice relations.

Definition 5. We call a choice relation $\triangleleft$ on $\mathcal{Q}(\mathcal{V})$ coherent if for all $O, O_{1}, O_{2}$ in $\mathcal{Q}$, all $u$ in $\mathcal{V}$ and all $\lambda$ in $\mathbb{R}_{>0}$ :

$\mathrm{S}_{1} . O \nless O$;

$\mathrm{S}_{2}$. if $u<v$ then $\{u\} \triangleleft\{v\}$;

$\mathrm{S}_{3}$. a. if $O_{1} \triangleleft O_{2}$ and $O_{2} \subseteq O$ then $O_{1} \triangleleft O$;

b. if $O_{1} \triangleleft O_{2}$ and $O \subset O_{1}$ then $O_{1} \backslash O \triangleleft O_{2} \backslash O$;

$\mathrm{S}_{4}$. a. if $O_{1} \triangleleft O_{2}$ then $\lambda O_{1} \triangleleft \lambda O_{2}$;

b. if $O_{1} \triangleleft O_{2}$ then $O_{1}+\{u\} \triangleleft O_{2}+\{u\}$.

We collect all coherent choice relations on $\mathcal{V}$ in the set $\overline{\mathcal{S}}$.

The following proposition collects a number of immediate properties of coherent choice relations 1

Proposition 4. The following statements hold for any coherent choice relation $\triangleleft$ :

(i) $\triangleleft$ is a strict partial order: it is irreflexive and transitive;

(ii) for all $O_{1}$ and $O_{2}$ in $\mathcal{Q}$, all $u$ in $\mathcal{V}$ and $\lambda$ in $\mathbb{R}_{>0}: O_{1} \triangleleft O_{2} \Leftrightarrow \lambda O_{1}+\{u\} \triangleleft \lambda O_{2}+\{u\}$.

The rationality axioms of Definitions 4 and 5 correspond:

Proposition 5. Given any coherent choice function $C, \triangleleft_{C}$ is a coherent choice relation. Conversely, given any coherent choice relation $\triangleleft, C_{\triangleleft}$ is a coherent choice function.

2.3. The 'is not more informative than' relation. Because we are interested in conservative reasoning with choice functions, we want to look for those implications of a given assessment that are as 'uninformative' as possible. To this end, we need to introduce some binary relation $\subseteq$ on the set $\mathcal{C}$ of all choice functions, having the specific interpretation of 'not more informative than', or, in other words, 'at least as uninformative as'.

Definition 6. Given two choice functions $C_{1}$ and $C_{2}$ in $\mathcal{C}$, we call $C_{1}$ not more informative than $C_{2}$-and we write $C_{1} \sqsubseteq C_{2}$-if $(\forall O \in \mathcal{Q}) C_{1}(O) \supseteq C_{2}(O)$.

This intuitive way of ordering choice functions is also used by Bradley [4] and Van Camp et al. [31]. The underlying idea is that a choice function is more informative when it consistently chooses more specifically — or more restrictively—amongst the available options. The corresponding notion for choice relations is as follows: given two choice relations $\triangleleft_{1}$ and $\triangleleft_{2}, \triangleleft_{1}$ is not more informative than $\triangleleft_{2}$-we write $\triangleleft_{1} \subseteq_{\mathcal{S}} \triangleleft_{2}$-if $\triangleleft_{1} \unlhd_{2}$. That is, choice relation $\triangleleft_{2}$ is at least as informative as $\triangleleft_{1}$ when every comparison made by $\triangleleft_{1}$ is also made by $\triangleleft_{2}$.

Since, by definition, $\subseteq$ is a product ordering of set inclusions, the following result is immediate [6].

Proposition 6. The structure $(\mathcal{C}$; 드) is a complete lattice:

(i) it is a partially ordered set, or poset, meaning that the binary relation $\sqsubseteq$ on $\mathcal{C}$ is reflexive, antisymmetric and transitive;

\footnotetext{
${ }^{1}$ See [16] for a similar result.
} 
(ii) for any subset $\mathcal{C}^{\prime}$ of $\mathcal{C}$, its infimum inf $\mathcal{C}^{\prime}$ and its supremum $\sup \mathcal{C}^{\prime}$ with respect to the ordering $\sqsubseteq$ exist in $\mathcal{C}$, and are given by $\inf ^{\prime}(O)=\cup_{C \in \mathcal{C}^{\prime}} C(O)$ and $\sup \mathcal{C}^{\prime}(O)=$ $\cap_{C \in \mathcal{C}^{\prime}} C(O)$ for all $O$ in $\mathcal{Q}$.

Moreover, $\left(\mathcal{S} ; \complement_{\mathcal{S}}\right)$ is a complete lattice as well.

The idea underlying these notions of infimum and supremum is that inf $\mathcal{C}^{\prime}$ is the most informative model that is not more informative than any of the models in $\mathcal{C}^{\prime}$, and $\sup \mathcal{C}^{\prime}$ the least informative model that is not less informative than any of the models in $\mathcal{C}^{\prime}$.

We will also consider the poset $(\overline{\mathcal{C}}$;드) of all coherent choice functions, where $\overline{\mathcal{C}} \subseteq \mathcal{C}$ inherits the partial order $\subseteq$ from $\mathcal{C}$. Similarly, in the poset $(\overline{\mathcal{S}} ; \sqsubseteq \mathcal{S})$ of all coherent choice relations, $\overline{\mathcal{S}}$ is assumed to inherit the partial order $\complement_{\mathcal{S}}$ from $\mathcal{S}$.

Also from an order-theoretic point of view, choice functions or choice relations are equivalent:

Proposition 7. The posets $(\mathcal{C} ;$ 드 $)$ and $\left(\mathcal{S} ; \check{S}_{\mathcal{S}}\right)$ are order isomorphic [6]: there is a map $f$ from $\mathcal{C}$ to $\mathcal{S}$ such that $C_{1} \sqsubseteq C_{2} \Leftrightarrow f\left(C_{1}\right) \sqsubseteq \mathcal{S} f\left(C_{2}\right)$ for all $C_{1}$ and $C_{2}$ in $\mathcal{C}$. Moreover, the posets $(\overline{\mathcal{C}} ; \sqsubseteq)$ and $(\overline{\mathcal{S}} ; \sqsubseteq \mathcal{S})$ are order isomorphic as well.

This proposition implies that we can regard $\sqsubseteq$ and $\subseteq \mathcal{S}$ essentially as the same partial orders, each defined on their respective domains, in the sense that each of the partial orders can be obtained from the other one just by renaming. Therefore, from now on, we will identify $\subseteq \mathcal{S}$ with $\sqsubseteq$, and only use the latter.

Proposition 8. $(\overline{\mathcal{C}} ; \sqsubseteq$ $)$ is a complete infimum-semilattice: $\overline{\mathcal{C}}$ is closed under arbitrary nonempty infima, so inf $\mathcal{C}^{\prime} \in \overline{\mathcal{C}}$ for any non-empty subset $\mathcal{C}^{\prime}$ of $\overline{\mathcal{C}}$.

As a result of Proposition 7. $(\overline{\mathcal{S}}$;드) is a complete infimum-semilattice as well.

This, for instance, allows us to do conservative inference with choice functions: if we consider that there is a coherent choice function that represents a subject's beliefs (coherent choices) and we can only tell that it belongs to a family $\left\{C_{i}: i \in I\right\}$, the conservative option is to consider its infimum $\inf _{i \in I} C_{i}$. This choice function is still guaranteed to be coherent, and as a consequence, it satisfies all the rationality requirements discussed above.

As we will show in Proposition 14 further on, there is a least informative coherent choice function, namely the infimum of all coherent choice functions: it is given by $C(O)=\{u \in$ $O:(\forall v \in O) u \nless v\}$ for all $O$ in $\mathcal{Q}$.

\section{THE RELATION WITH SETS OF DESIRABLE OPTIONS}

In general, a choice function cannot be characterised using only pairwise comparisons of options, meaning that a binary relation on options does not uniquely determine a choice function. In this section, we study a special class of choice functions that are determined by their restrictions to option sets of cardinality two.

3.1. Sets of desirable options. Sets of desirable options are a(n obvious and immediate) generalisation of sets of desirable gambles. Gambles are real-valued maps on a possibility space $\mathcal{X}$, interpreted as uncertain rewards. They can be seen as vectors in the vector space $\mathbb{R}^{\mathcal{X}}$. Here we generalise this notion by looking at a general (abstract) vector space $\mathcal{V}$ of (abstract) options, rather than gambles, provided with a vector ordering $\leq$; see also Section 2.2.1. We will see that sets of desirable options amount to a pairwise comparison of options and therefore correspond to a special kind of choice functions.

A set of desirable options $D$ is simply a subset of the vector space of options $\mathcal{V}$-its interpretation will be that it contains those options that some subject strictly prefers to the 
status quo 0 . We collect all possible such sets of desirable options in the set $\mathcal{D}$. As we did for choice functions, we pay special attention to coherent sets of desirable options. The following is an immediate generalisation of existing coherence definitions [7, 10, 11, 19] from gambles to abstract options.

Definition 7. A set of desirable options $D$ is called coherent if for all $u$ and $v$ in $\mathcal{V}$ and $\lambda$ in $\mathbb{R}_{>0}$ :

$\mathrm{D}_{1} .0 \notin D ;$

$\mathrm{D}_{2} . \mathcal{V}_{>0} \subseteq D$;

$\mathrm{D}_{3}$. if $u \in D$ then $\lambda u \in D$;

$\mathrm{D}_{4}$. if $u, v \in D$ then $u+v \in D$.

We let $\overline{\mathcal{D}}$ denote the set of all coherent sets of desirable options.

Axioms $\mathrm{D}_{3}$ and $\mathrm{D}_{4}$ turn coherent sets of desirable options $D$ into convex cones-meaning that $\operatorname{posi}(D)=D$. They include the positive options due to Axiom $\mathrm{D}_{2}$, but not the zero option due to Axiom $\mathrm{D}_{1}$ As an immediate consequence, their intersection with $\mathcal{V}_{<0}:=-\mathcal{V}_{>0}$ is empty. As usual, we may associate with the convex cone $D$ a strict partial order $\varangle-$ called preference relation - on $\mathcal{V}$, by letting $u \triangleleft v \Leftrightarrow 0 \triangleleft v-u \Leftrightarrow v-u \in D$, leading to $D=\{u \in \mathcal{V}: 0 \triangleleft u\}$ [11, 19]. The connection between this order and the one associated to choice functions will be clarified in Section 3.3 .

3.2. The 'is not more informative than' relation. Sets of desirable options can be ordered according to a 'not more informative than' relation, analogously to the ordering introduced for choice functions.

Definition 8. Given two sets of desirable options $D_{1}, D_{2}$ in $\mathcal{D}$, we call $D_{1}$ not more informative than $D_{2}$ when $D_{1} \subseteq D_{2}$.

Because the ordering of sets of desirable options $\subseteq$ is just set inclusion, it is a partial ordering on $\mathcal{D}$, and the poset $(\mathcal{D} ; \subseteq)$ is a complete lattice, with supremum operator $\bigcup$, and infimum operator $\cap$. Next we investigate the structure of the set of all coherent sets of desirable options:

Proposition 9. $(\overline{\mathcal{D}} ; \subseteq)$ is a complete infimum-semilattice, or alternatively, $\overline{\mathcal{D}}$ is an intersection structure - closed under arbitrary non-empty intersections. The least informative (smallest) set of desirable options $D_{\mathrm{v}}$ is given by $D_{\mathrm{v}}:=\mathcal{V}_{>0}$.

We will refer to $D_{\mathrm{v}}$ as the vacuous set of desirable options.

It will be useful to also consider the maximally informative, or maximal, coherent sets of desirable options ${ }^{2}$ They are the undominated elements of the complete infimum-semilattice $(\overline{\mathcal{D}} ; \subseteq)$; we collect them into a set $\hat{\mathcal{D}}$ :

$$
\hat{\mathcal{D}}:=\left\{D \in \overline{\mathcal{D}}:\left(\forall D^{\prime} \in \overline{\mathcal{D}}\right)\left(D \subseteq D^{\prime} \Rightarrow D=D^{\prime}\right)\right\} .
$$

First, we prove a useful proposition that will allow us to characterise these maximal elements very elegantly.

Proposition 10. Given any coherent set of desirable options $D$ and any non-zero option $u \notin D$, posi $(D \cup\{-u\})$ is a coherent set of desirable options. As a consequence, a coherent set of desirable options $D$ is maximal if and only if

$$
(\forall u \in \mathcal{V} \backslash\{0\})(u \in D \text { or }-u \in D) \text {. }
$$

\footnotetext{
${ }^{2}$ The discussion in the rest of this section is based on similar discussions about sets of desirable gambles [5. 11 20]. We repeat the details here mutatis mutandis to make the paper more self-contained.
} 
Next we show that the set of all coherent sets of desirable options is dually atomic, meaning that any coherent set of desirable options is the infimum of its non-empty set of dominating maximal coherent sets of desirable options:

Proposition 11. For any coherent set of desirable options $D$, its set of dominating maximal coherent sets of desirable options $\hat{\mathcal{D}}_{D}:=\{\hat{D} \in \hat{\mathcal{D}}: D \subseteq \hat{D}\}$ is non-empty. Moreover, $D=\inf \hat{\mathcal{D}}_{D}$.

3.3. Connection between choice functions and sets of desirable options. We now set out to establish a connection between choice functions and sets of desirable options.

Definition 9. Given a choice function $C$, we say that an option $v$ is chosen over some option $u$ whenever $u \notin C(\{u, v\})$, or equivalently, whenever $\{u\} \triangleleft_{C}\{v\}$. Similarly, given a set of desirable options $D$, we say that an option $v$ is preferred to some option $u$ whenever $v-u \in D$, or equivalently, $u \triangleleft v$. We call a choice function $C$ and a set of desirable options $D$ compatible when

$$
u \notin C(\{u, v\}) \Leftrightarrow v-u \in D \Leftrightarrow u \triangleleft v \text { for all } u, v \in \mathcal{V} .
$$

Compatibility means that the behaviour of the choice function restricted to pairs of options reflects the behaviour of the set of desirable options ${ }^{3}$ So, a choice function $C$ will have at most one compatible set of desirable options, whereas conversely, a set of desirable options $D$ may have many compatible choice functions: compatibility only directly influences the behaviour of a choice function on pairs.

The definition of compatibility with desirability is clearer in terms of the relations: a choice relation $\triangleleft$ is compatible with some preference relation $\triangleleft$ if $\{u\} \triangleleft\{v\} \Leftrightarrow u \triangleleft v$ for all $u$ and $v$ in $\mathcal{Q}$. Loosely speaking, $\triangleleft$ and $\triangleleft$ are compatible if $\triangleleft$ lifts $\triangleleft$ from options to sets of options. This observation also sheds light on the necessity of our Axiom $\mathrm{C}_{2}$ which Seidenfeld et al. [27] use a weakened version of. Indeed, if a choice relation does not satisfy Axiom $\mathrm{C}_{2}$ then necessarily $0 \nless u$ for some $0<u$, contradicting Axiom $\mathrm{D}_{2}$

3.3.1. From choice functions to desirability. We begin by studying the properties of the set of desirable options compatible with a given coherent choice function. Since compatibility is related to the restriction of the choice function to pairwise comparison, it is not surprising that each choice function has a unique compatible set of desirable options:

Proposition 12. For any coherent choice function $C$ in $\overline{\mathcal{C}}$, the unique compatible coherent set of desirable options $D_{C}$ is given by $D_{C}:=\{u \in \mathcal{V}: 0 \notin C(\{0, u\})\}=\left\{u \in \mathcal{V}:\{0\} \triangleleft_{C}\{u\}\right\}$.

Example 1. Consider $\mathcal{V}=\mathbb{R}^{2}$, and let us denote the two components of any vector $u$ in $\mathbb{R}^{2}$ by $u_{1}$ and $u_{1}$, so $u=\left(u_{1}, u_{2}\right)$. Let $C$ be the choice function given by

$$
C(O):=\left\{u \in O: \text { there is no } v \in O \text { such that } v_{1}+v_{2}>u_{1}+u_{2}\right\} .
$$

It is easy to check that $C$ is a coherent choice function-it is an example of what we will call an $E$-admissible choice function later. And

$$
D_{C}=\left\{u \in \mathcal{V}: u_{1}+u_{2}>0\right\}
$$

is its associated coherent set of desirable options.

\footnotetext{
${ }^{3}$ See Seidenfeld et al. [25] for an axiomatisation of imprecise preferences in the context of binary comparisons of horse lotteries, rather than gambles.
} 
3.3.2. From desirability to choice functions. We collect in $\overline{\mathcal{C}}_{D}$ all the coherent choice functions that are compatible with a given coherent set of desirable options $D$ :

$$
\begin{aligned}
\overline{\mathcal{C}}_{D} & :=\{C \in \overline{\mathcal{C}}:(\forall u, v \in \mathcal{V})(v \notin C(\{u, v\}) \Leftrightarrow u-v \in D)\} \\
& =\left\{C \in \overline{\mathcal{C}}: D_{C}=D\right\} .
\end{aligned}
$$

The correspondence between choice functions and sets of desirable options can be manyto-one, in the sense that several different coherent choice functions may be compatible with the same coherent set of desirable options. An important role is played by the most uninformative of them:

Proposition 13. Given a coherent set of desirable options D, the infimum-most uninformative element-inf $\overline{\mathcal{C}}_{D}$ of its set of compatible coherent choice functions $\overline{\mathcal{C}}_{D}$ is the coherent choice function $C_{D}$, defined by

$$
\begin{aligned}
C_{D}(O): & :=\{u \in O:(\forall v \in O) v-u \notin D\} \\
& =\{u \in O:(\forall v \in O) u \nless v\} \text { for all } O \text { in } \mathcal{Q} .
\end{aligned}
$$

The coherent choice function $C_{D}$ is the least informative choice function that is compatible with a coherent set of desirable options $D$ : it is based on the binary ordering represented by $D$ and nothing else. As we will see in Proposition 19 , there typically are other coherent choice functions $C$ compatible with $D$, but they encode more information than just the binary ordering represented by $D$. Proposition 13 is especially interesting because it shows that the most conservative choice function based on a strict partial order of options, is the choice function based on maximalit) $y_{\text {- }}^{4}$ the one that selects the undominated options under the strict partial order $\triangleleft$ associated with a coherent set of desirable options $D$. Any choice function that is based on maximality under such a strict partial order is coherent.

Proposition 13 can also be interpreted in terms of choice relations: given a coherent set of desirable gambles $D$ - or a coherent preference relation $\triangleleft$ - the most uninformative choice relation $\triangleleft_{D}$ that is compatible with it, is by Proposition 7 equal to $\triangleleft_{C}$, and determined by

$$
O_{1} \triangleleft_{D} O_{2} \Leftrightarrow\left(\forall u \in O_{1}\right)\left(\exists v \in O_{2}\right) u \triangleleft v \text {, for all } O_{1} \text { and } O_{2} \text { in } \mathcal{Q} \text {. }
$$

This shows that the partial order $\triangleleft_{D}$ is simply the result of lifting $\triangleleft$ from elements to sets.

Example 2. Let $D$ be the coherent set of desirable options given by Equation (7). Its associated coherent choice function $C_{D}$ is given by

$$
C(O):=\left\{u \in O: \text { there is no } v \in O \text { such that } v_{1}+v_{2}>u_{1}+u_{2}\right\},
$$

so it coincides with the choice function given by Equation 6 .

Although in this particular example the two procedures coincide, there is in general more than one coherent choice function that is compatible with a coherent set of desirable options. In other words, a coherent choice function is not uniquely determined by its restriction to binary comparisons; we will give an example in Example 6 further on.

Proposition 8 guarantees that there is a unique smallest-least informative-coherent choice function. We will call it the vacuous choice function, and denote it by $C_{\mathrm{v}}$.

Proposition 14. The vacuous choice function $C_{\mathrm{v}}$ is given by $C_{\mathrm{v}}(O)=C_{D_{\mathrm{v}}}(O)=\{u \in O$ : $(\forall v \in O) u \nless v\}$ for all $O$ in $\mathcal{Q}$. It selects from any set of options the ones that are undominated under the strict vector ordering $<$.

\footnotetext{
${ }^{4}$ Note that maximility here refers to the optimality decision criterion [30 34], and it does not mean that the set of desirable options $D$ is a maximal one, in the sense considered in Section 3.2
} 
Similarly, the vacuous choice relation $\triangleleft_{\mathrm{v}}$ is equal to $\triangleleft_{C_{\mathrm{v}}}$ by Proposition 7, and is determined by $O_{1} \triangleleft_{\mathrm{v}} O_{2} \Leftrightarrow\left(\forall u \in O_{1}\right)\left(\exists v \in O_{2}\right) u<v$ for all $O_{1}$ and $O_{2}$ in $\mathcal{Q}$.

Example 3. Consider, as a simple example, the case that the vector ordering is total, meaning that for any $u, v$ in $\mathcal{V}$, either $u<v, v<u$ or $u=v$. It then follows from Proposition 14 that, for any coherent choice function $C, C(O) \subseteq C_{\mathrm{v}}(O)=\max O$ for all $O \in \mathcal{Q}$, where $\max O$ is the unique largest element of the finite option set $O$ according to the strict total ordering <. But then Axiom $\mathrm{C}_{1}$ guarantees that $C(O)=C_{\mathrm{v}}(O)=\max O$ for all $O \in \mathcal{Q}$, so $C_{\mathrm{v}}$ is the only coherent choice function.

3.3.3. Properties of the relation between choice functions and desirability. Since sets of desirable options represent only pairwise comparison, and are therefore generally less expressive than choice functions, we expect that going from a choice function to a compatible set of desirable options leads to a loss of information, whereas going the opposite route does not. This is confirmed by Propositions 15 and 16 and in particular by their Corollary 17.

Proposition 15. Consider any set of coherent choice functions $\mathcal{C}^{\prime} \subseteq \overline{\mathcal{C}}$. Then $D_{\inf \mathcal{C}^{\prime}}=\inf \left\{D_{C}\right.$ : $\left.C \in \mathcal{C}^{\prime}\right\}$ and $C_{\inf \left\{D_{C}: C \in \mathcal{C}^{\prime}\right\}} \sqsubseteq \inf \mathcal{C}^{\prime}$, and therefore also $C_{D_{\text {inf }}} \sqsubseteq \inf \mathcal{C}^{\prime}$.

Definition 10. For any set of coherent sets of desirable options $\mathcal{D}^{\prime} \subseteq \overline{\mathcal{D}}$, we define the 'infimum of maximality' choice function as $C_{\mathcal{D}^{\prime}}:=\inf \left\{C_{D}: D \in \mathcal{D}^{\prime}\right\}$.

Proposition 16. Consider any set of coherent sets of desirable options $\mathcal{D}^{\prime} \subseteq \overline{\mathcal{D}}$. Then $D_{C_{\mathcal{D}^{\prime}}}=\inf \mathcal{D}^{\prime}$. Moreover, $C_{\mathrm{inf} \mathcal{D}^{\prime}} \sqsubseteq C_{\mathcal{D}^{\prime}}$.

This result also helps to highlight that coherent choice functions are indeed more informative than coherent sets of desirable options, in the sense that two different coherent choice functions $\left(C_{\mathrm{inf} \mathcal{D}^{\prime}}\right.$ and $\left.C_{\mathcal{D}^{\prime}}\right)$ may determine the same coherent set of desirable options $\left(\inf \mathcal{D}^{\prime}\right)$ when restricted to option sets of cardinality two. Thus, we need to move to this richer model in order to fully represent the beliefs encompassed by $\left\{C_{D}: D \in \mathcal{D}^{\prime}\right\}$. Moreover, this happens even if each of the choice functions in the set $\mathcal{D}^{\prime}$ is fully determined by a coherent set of desirable options.

From these two results we immediately deduce the following:

Corollary 17. Consider any coherent set of desirable options $D \in \overline{\mathcal{D}}$ and any coherent choice function $C \in \overline{\mathcal{C}}$. Then $D=D_{C_{D}}$ and $C_{D_{C}} \subseteq C$.

If we consider the maps

$$
\begin{aligned}
& D_{\bullet}: \mathcal{D} \rightarrow \mathcal{C}: D \mapsto C_{D} \text { as defined by Proposition } 12 \\
& C_{\bullet}: \mathcal{C} \rightarrow \mathcal{D}: C \mapsto D_{C} \text { as defined in Equation }(8),
\end{aligned}
$$

then Corollary 17 essentially states that $D_{\bullet} \circ C_{\bullet}=\mathrm{id}$, the identity map, while $\left(C_{\bullet} \circ D_{\bullet}\right)(C)$ 드 $C$ for all $C$ in $\mathcal{C}$. This observation helps us interpret Propositions 15 and 16 using the commuting diagrams for Proposition 15

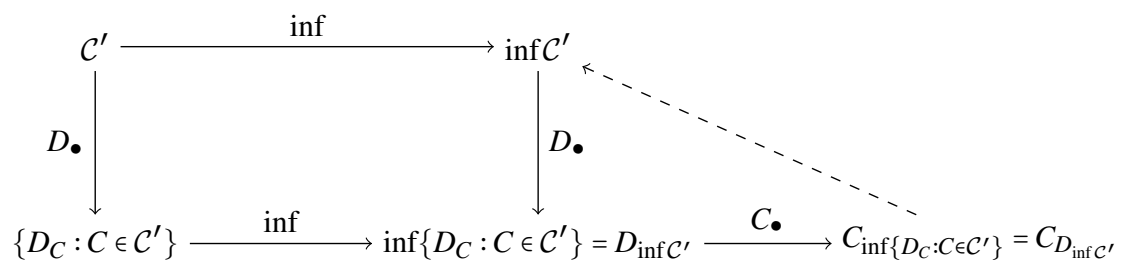

and for Proposition 16 . 


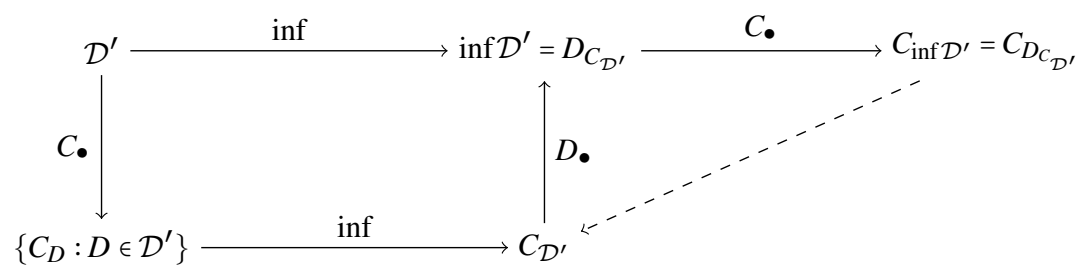

Full lines indicate the maps $\left(D_{\bullet}, C_{\bullet}\right.$ or inf), while dashed lines indicate an "is not more informative than" ( $\subseteq$ for choice functions, $\subseteq$ for sets of desirable gambles) relation.

Example 5 in Section 4 further on will show that the inequalities in these results can be strict; this does not seem surprising for the inequality of Proposition 15, but is perhaps less intuitively obvious for the one in Proposition 16

\section{OTHER TYPES OF COHERENT CHOICE FUNCTIONS}

Given a coherent set of desirable options, we can define a coherent choice function by selecting undominated elements as in Equation (8). However, these are not the only possible coherent choice functions: for instance, any infimum of such coherent choice functions is still coherent.

Proposition 18. Consider any set of coherent sets of desirable options $\mathcal{D}^{\prime} \subseteq \overline{\mathcal{D}}$, then $C_{\mathcal{D}^{\prime}}$ is a coherent choice function.

This proposition gives a procedure to define coherent choice functions complying with a sensitivity analysis interpretation, where the underlying uncertainty model is that of coherent sets of desirable options: we may consider a set $\mathcal{D}^{\prime}$ of possible models and then the set of coherent choice functions they determine; by taking the infimum of this set we end up choosing those options that are considered acceptable by at least one of the possible models.

Example 4. Consider again $\mathcal{V}=\mathbb{R}^{2}$, and let $D_{1}, D_{2}$ the coherent sets of desirable options given by

$$
D_{1}=\left\{u \in \mathbb{R}^{2}: u(x)+u(y)>0\right\} \text { and } D_{2}=\left\{u \in \mathbb{R}^{2}: 2 u(x)+u(y)>0\right\} .
$$

Then the choice function $C=\inf \left\{C_{D_{1}}, C_{D_{2}}\right\}$ is coherent. If we consider for instance the option set $O=\{(4,-4),(2,-1),(0,0),(-1,2)\}$, we obtain

$$
C(O)=\inf \left\{C_{D_{1}}(O), C_{D_{2}}(O)\right\}=\{(2,-1),(-1,2),(4,-4)\} .
$$

The first two options are elements of $C_{D_{1}}(O)$ and the last one is an element of $C_{D_{2}}(O)$.

As special cases, we may consider 'infimum of maximality' choice functions where some additional condition is imposed on the coherent sets of desirable options. We will investigate two such situations in this section, although other are possible. In Definition 11 . we focus only on sets of maximal coherent sets of desirable options, that we introduced in Section 3.2 Their interest lies in the fact that they are related to mass functions, as we discussed quite thoroughly in [5, 11, 20] in the context of gambles.

Definition 11. If $\mathcal{D}^{\prime} \subseteq \hat{\mathcal{D}}$ is a set of maximal coherent set of desirable options, the coherent choice function $C_{\mathcal{D}^{\prime}}$ is called $M$-admissible. We will also denote it by $C_{\mathcal{D}^{\prime}}^{\mathrm{M}}$ as a reminder that the infimum is taken over maximal sets.

In particular, we can consider the M-admissible choice functions for the set $\mathcal{D}^{\prime}=\hat{\mathcal{D}}_{D}$ of all maximal coherent set of desirable options that include a coherent set of desirable options $D$. 
In order not to overburden the notation, we let

$$
C_{D}^{\mathrm{M}}:=C_{\hat{\mathcal{D}}_{D}}^{\mathrm{M}}=\inf \left\{C_{\hat{D}}: \hat{D} \in \hat{\mathcal{D}} \text { and } D \subseteq \hat{D}\right\},
$$

and similarly to what we did before, we introduce the map

$$
C_{\bullet}^{\mathrm{M}}: \mathcal{D}^{\prime} \rightarrow \mathcal{C}^{\prime}: D \mapsto C_{D}^{\mathrm{M}} \text { as defined in Equation (9). }
$$

The following result can be regarded as a particular case of Proposition 16 , where all the coherent sets of desirable options are maximal ones. As we have seen there too, the diagram below commutes if we focus on sets of desirable options, but this is not longer the case if we consider the more informative model of coherent choice functions.

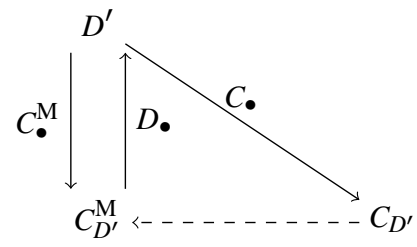

Proposition 19. Consider any coherent set of desirable options $D^{\prime} \in \overline{\mathcal{D}}$. Then $D^{\prime}=D_{C_{D^{\prime}}^{M}}$ and $C_{D^{\prime}} \sqsubseteq C_{D^{\prime}}^{M}$.

The inequality in Proposition 19 can be strict—meaning that $C_{D^{\prime}} \sqsubset C_{D^{\prime}}^{\mathrm{M}}$ for some coherent set of desirable options $D^{\prime}$ - as we will show in Example 6 below.

As another special case, we consider choice functions associated with Levi]'s notion of Eadmissibility [17, Chapter 5]. They are based on a non-empty set of mass functions. Consider a finite possibility space $\mathcal{X}$, and maps from $\mathcal{X}$ to $\mathbb{R}$ (the options are gambles now), forming the vector space $\mathcal{V}=\mathbb{R}^{\mathcal{X}}$ of finite dimension $|\mathcal{X}|$. The vector ordering $\leq$ we associate with this vector space of options is the pointwise ordering of real maps: $u \leq v \Leftrightarrow(\forall x \in \mathcal{X}) u_{x} \leq v_{x}$, where, for instance, $u_{x}=u(x)$ is the $x$-component of the option $u$. We call any map $p: \mathcal{X} \rightarrow \mathbb{R}$ with $(\forall x \in \mathcal{X}) p(x) \geq 0$ and $\sum_{x \in \mathcal{X}} p(x)=1 \mathrm{a}$ (probability) mass function, and we associate an expectation $\mathrm{E}_{p}$ with $p$ by letting $\mathrm{E}_{p}(u):=\sum_{x \in \mathcal{X}} p(x) u_{x}$ for all $u$ in $\mathcal{V}$. We collect in $\Sigma_{\mathcal{X}}$ all the mass functions on $\mathcal{X}$.

With a mass function $p$, we associate a set of desirable options

$$
D_{p}:=\mathcal{V}_{>0} \cup\left\{u \in \mathcal{V}: \mathrm{E}_{p}(u)>0\right\}
$$

and a choice function $C_{p}$ defined for all $O$ in $\mathcal{Q}$ by

$$
C_{p}(O):=\left\{u \in O:(\forall v \in O)\left(\mathrm{E}_{p}(u) \geq \mathrm{E}_{p}(v) \text { and } u \nless v\right)\right\} .
$$

Proposition 20. The set of desirable options $D_{p}$ and the choice function $C_{p}$ are coherent and compatible, and moreover $C_{p}=C_{D_{p}}$.

This result allows us to introduce the following, second special case of 'infimum of maximality' choice functions.

Definition 12. With any non-empty set of mass functions $K_{5}^{5}$ we associate the corresponding $E$-admissible choice function $C_{K}^{\mathrm{E}}:=\inf \left\{C_{p}: p \in K\right\}=C_{\left\{D_{p}: p \in K\right\}}$.

\footnotetext{
${ }^{5}$ Although Levi s notion of E-admissibility was originally concerned with convex closed sets of mass functions [17 Chapter 5], we impose no such requirement here on the set $K$.
} 
Proposition 21. Given any non-empty set of mass functions $K$, we have for all $O$ in $\mathcal{Q}$ that

$$
C_{K}^{E}(O)=\left\{u \in O:(\exists p \in K) \mathrm{E}_{p}(u) \in \underset{v \in O}{\arg \max } \mathrm{E}_{p}(v)\right\} \cap C_{\mathrm{v}}(O) .
$$

The following proposition establishes a connection between M-admissible and E-admissible choice functions.

Proposition 22. For any non-empty set of mass functions $K, C_{K}^{E} \sqsubseteq C_{\hat{\mathcal{D}}_{K}}^{M}$, where $\hat{\mathcal{D}}_{K}:=$ $\cup_{p \in K} \hat{\mathcal{D}}_{D_{p}} \subseteq \hat{\mathcal{D}}$.

The key for this result is that, for any mass function $p$, there is in general more than one coherent set of desirable options $D$ that is associated with it by means of the formula

$$
\mathrm{E}_{p}(u)=\sup \{\mu: u-\mu \in D\} .
$$

This is why in general coherent sets of desirable options are more informative than (sets of) mass functions as a belief model. Among all the coherent sets of desirable options satisfying Equation (12) with respect to a fixed $p$, the least informative one is the one given by Equation (10), which is usually referred to as the set of strictly desirable gambles associated with $p$ within the imprecise probability literature. This in turn gives rise to a coherent choice function that will be less informative than one determined by a maximal set of options that is compatible with $p$ by means of Equation 12 .

Thus, the choice between E-admissible and M-admissible coherent choice functions can be made by considering our attitude towards imprecision, that determines the use of strictly desirable or maximal sets of options: the former are as conservative as possible, and make a choice only when it is implied by the probability mass function $p$; while the latter are as informative as it can be considering the axioms of coherence and the probability mass function $p$.

The following examples show why choice functions are more powerful than sets of desirable options as uncertainty representations, and elucidate the difference between Eadmissible and $\mathrm{M}$-admissible choice functions.

Example 5. Consider the situation where you have a coin with two identical sides of unknown type ${ }^{6}$ either both sides are heads $(\mathrm{H})$, or both sides are tails $(\mathrm{T})$. The random variable that represents the outcome of a coin flip assumes a value in the finite possibility space $\mathcal{X}:=\{\mathrm{H}, \mathrm{T}\}$. The options we consider are gambles: real-valued functions on $\mathcal{X}$, which constitute the two-dimensional vector space $\mathbb{R}^{\mathcal{X}}$, ordered by the pointwise order. We model this situation using (a) coherent sets of desirable options, (b) M-admissible choice functions, and (c) E-admissible choice functions. In all three cases we start from two simple models: one that describes practical certainty of $\mathrm{H}$ and another that describes practical certainty of $\mathrm{T}$, and we take their infimum - the most informative model that is still at most as informative as either-as a candidate model for the coin problem.

For (a), we use two coherent sets of desirable options $D_{\mathrm{H}}$ and $D_{\mathrm{T}}$, expressing practical certainty of $\mathrm{H}$ and $\mathrm{T}$, respectively, given by the maximal sets of desirable options $D_{\mathrm{H}}:=$ $\mathcal{V}_{>0} \cup\left\{u \in \mathcal{V}: u_{\mathrm{H}}>0\right\}$ and $D_{\mathrm{T}}:=\mathcal{V}_{>0} \cup\left\{u \in \mathcal{V}: u_{\mathrm{T}}>0\right\}$, where $u_{\mathrm{H}}$ and $u_{\mathrm{T}}$ denote the values of the gamble $u$ in $\mathrm{H}$ and $\mathrm{T}$, respectively. The model for the coin with two identical sides is then $D_{\mathrm{H}} \cap D_{\mathrm{T}}=\mathcal{V}_{\succ 0}$. This vacuous model $D_{\mathrm{v}}$ is incapable of distinguishing between this situation and the one where we are completely ignorant about the coin.

\footnotetext{
${ }^{6}$ The example can be trivially reformulated to consider a random variable taking values in a binary possibility space, with only one of those elements occurring; however we think the use of a coin adds some intuition and helps in particular when indifference in Section 5 later on.
} 
For an approach (b) that distinguishes between these two situations, we draw inspiration from Proposition 15 , instead of working with the sets of desirable options themselves, we move to the corresponding choice functions $C_{\mathrm{H}}:=C_{D_{\mathrm{H}}}$ and $C_{\mathrm{T}}:=C_{D_{\mathrm{T}}}$, where

$$
\begin{aligned}
C_{\mathrm{H}}(O) & =\left\{u \in O:(\forall v \in O) v-u \notin D_{\mathrm{H}}\right\}=\arg \max \left\{u_{\mathrm{H}}: u \in O\right\} \cap C_{\mathrm{V}}(O) \\
& =\arg \max \left\{v_{\mathrm{T}}: v \in \arg \max \left\{u_{\mathrm{H}}: u \in O\right\}\right\} \\
C_{\mathrm{T}}(O) & =\arg \max \left\{u_{\mathrm{T}}: u \in O\right\} \cap C_{\mathrm{V}}(O) \\
& =\arg \max \left\{v_{\mathrm{H}}: v \in \arg \max \left\{u_{\mathrm{T}}: u \in O\right\}\right\}
\end{aligned}
$$

for all $O$ in $\mathcal{Q}$. We infer that $\left|C_{\mathrm{H}}(O)\right|=\left|C_{\mathrm{T}}(O)\right|=1$ for every $O$ in $\mathcal{Q}$ : for instance in the case of $C_{\mathrm{H}}$, note that amongst all the options attaining the maximum value on heads, exactly one of them is undominated. The M-admissible choice function we are looking for is $C_{\left\{D_{\mathrm{H}}, D_{\mathrm{T}}\right\}}^{\mathrm{M}}=\inf \left\{C_{\mathrm{H}}, C_{\mathrm{T}}\right\}$, which selects at most two options from each option set. It is given by

$$
C_{\left\{D_{\mathrm{H}}, D_{\mathrm{T}}\right\}}^{\mathrm{M}}(O)=\left(\arg \max \left\{u_{\mathrm{H}}: u \in O\right\} \cup \arg \max \left\{u_{\mathrm{T}}: u \in O\right\}\right) \cap C_{\mathrm{v}}(O)
$$

for all $O$ in $\mathcal{Q}$, and differs from the vacuous choice function $C_{\mathrm{v}}$. Indeed, consider the particular option set $O=\{u, v, w\}$, where $u=(1,0), v=(0,1)$ and $w=(1 / 2,1 / 2)$. Then $C_{\left\{D_{\mathrm{H}}, D_{\mathrm{T}}\right\}}^{\mathrm{M}}(O)=\{u, v\} \neq O=C_{\mathrm{V}}(O)$.

For (c), the set of mass functions $K$ consists of the two degenerate mass functions: $K=\left\{p_{\mathrm{H}}, p_{\mathrm{T}}\right\}$, where $p_{\mathrm{H}}=(1,0)$ and $p_{\mathrm{T}}=(0,1)$. The corresponding expectations $\mathrm{E}_{\mathrm{H}}:=\mathrm{E}_{p_{\mathrm{H}}}$ and $\mathrm{E}_{\mathrm{T}}:=\mathrm{E}_{p_{\mathrm{T}}}$ satisfy $\mathrm{E}_{\mathrm{H}}(u)=u_{\mathrm{H}}$ and $\mathrm{E}_{\mathrm{T}}(u)=u_{\mathrm{T}}$ for all $u$ in $\mathcal{V}$. So we see that $C_{p_{\mathrm{H}}}=C_{\mathrm{H}}$ and $C_{p_{\mathrm{T}}}=C_{\mathrm{T}}$, and therefore this approach leads to the same choice function as the previous one: $C_{\left\{p_{\mathrm{H}}, p_{\mathrm{T}}\right\}}^{\mathrm{E}}=C_{\left\{D_{\mathrm{H}}, D_{\mathrm{T}}\right\}}^{\mathrm{M}}=\inf \left\{C_{\mathrm{H}}, C_{\mathrm{T}}\right\}$.

The example above shows that the correspondence between desirability and choice functions is not a complete inf-homomorphism, as illustrated also at the end of Section 3 .

Example 6. In this example, we illustrate the difference between E-admissible and Madmissible choice functions. We consider the same finite possibility space $\mathcal{X}:=\{\mathrm{H}, \mathrm{T}\}$ as in Example 5, with the same option space and vector ordering. For both E-admissibility and M-admissibility, we each time consider the least informative choice functions: the E-admissible choice function $C_{\Sigma_{\mathcal{X}}}^{\mathrm{E}}$ associated with set of all mass functions $K=\Sigma_{\mathcal{X}}$, and the M-admissible choice function $C_{D_{\mathrm{v}}}^{\mathrm{M}}$ associated with the set of all maximal sets of desirable options $\hat{\mathcal{D}}_{D_{\mathrm{v}}}=\hat{\mathcal{D}}$. Since $C_{\Sigma_{\mathcal{X}}}^{\mathrm{E}}$ and $C_{D_{\mathrm{v}}}^{\mathrm{M}}$ are the most conservative E-admissible, respectively $\mathrm{M}$-admissible choice functions, we wonder about their relation, as well as their relation with the vacuous choice function $C_{\mathrm{v}}$. We find that $C_{\Sigma_{\mathcal{X}}}^{\mathrm{E}} \sqsubseteq C_{D_{\mathrm{v}}}^{\mathrm{M}}$. Indeed, consider any $O$ in $\mathcal{Q}$ and any $u$ in $C_{D_{\mathrm{v}}}^{\mathrm{M}}(O)$, being equivalent to $0 \in C_{D_{\mathrm{v}}}^{\mathrm{M}}(O-\{u\})$ by Proposition 3 (i) whence

$$
u \in C_{D_{\mathrm{v}}}^{\mathrm{M}}(O) \Leftrightarrow(\exists \hat{D} \in \hat{\mathcal{D}})(\forall v \in O-\{u\}) v \notin \hat{D} \Leftrightarrow(\exists \hat{D} \in \hat{\mathcal{D}}) O-\{u\} \cap \hat{D}=\varnothing .
$$

Since for every $\hat{D}$ in $\hat{\mathcal{D}}$, there is some mass function $p$ in $\Sigma_{\mathcal{X}}$ such that $D_{p} \subseteq \hat{D}$ [it suffices to consider the mass function $p$ corresponding with $\hat{D}$ ], we find that

$$
u \in C_{D_{\mathrm{v}}}^{\mathrm{M}}(O) \Rightarrow\left(\exists p \in \Sigma_{\mathcal{X}}\right) O-\{u\} \cap D_{p}=\varnothing \Leftrightarrow\left(\exists p \in \Sigma_{\mathcal{X}}\right) u \in C_{D_{p}}(O) .
$$

or in other words, $u \in C_{D_{\mathrm{v}}}^{\mathrm{M}}(O)$ implies that $u \in C_{\Sigma_{\mathcal{X}}}^{\mathrm{E}}(O)$, whence $C_{D_{\mathrm{v}}}^{\mathrm{M}}(O) \subseteq C_{\Sigma_{\mathcal{X}}}^{\mathrm{E}}(O)$. By the definition of the vacuous choice function, we have as an intermediate result that $C_{\mathrm{v}} \sqsubseteq C_{\Sigma_{\mathcal{X}}}^{\mathrm{E}}$ $C_{D_{\mathrm{v}}}^{\mathrm{M}}$.

Both inequalities are strict; to show that $C_{\mathrm{v}} \neq C_{\Sigma_{\mathcal{X}}}^{\mathrm{E}}$, consider the option set $O:=\{0, u, v\}$, where $u=(1,-1 / 4)$ and $v=(-1 / 4,1)$. Because all options in $O$ are pointwise undominated in 
$O$, we find that $C_{\mathrm{v}}(O)=O$, and in particular, that $0 \in C_{\mathrm{v}}(O)$. On the other hand, it follows from Proposition 21 that

$$
0 \in C_{\Sigma_{\mathcal{X}}}^{\mathrm{E}}(O) \Leftrightarrow\left(\exists p \in \Sigma_{\mathcal{X}}\right)\left(0 \geq p(\mathrm{H})-\frac{1}{4} p(\mathrm{~T}) \text { and } 0 \geq-\frac{1}{4} p(\mathrm{H})+p(\mathrm{~T})\right),
$$

which would imply that $\left(\exists p \in \Sigma_{\mathcal{X}}\right)\left(0 \geq \frac{3}{4} p(\mathrm{H})+\frac{3}{4} p(\mathrm{~T})\right)$, which is impossible.

More importantly, we also have that $C_{\Sigma_{\mathcal{X}}}^{\mathrm{E}} \neq C_{D_{\mathrm{v}}}^{\mathrm{M}}$. Consider the option set $O:=\{0, u,-u\}$, where $u=(1,-1)$. Then for the specific mass function $p:=(1 / 2,1 / 2) \in \Sigma_{\mathcal{X}}$, we find that $0 \in C_{p}(O)$, whence $0 \in C_{\Sigma_{\mathcal{X}}}^{\mathrm{E}}(O)$. To show that $0 \in C_{p}^{\mathrm{E}}(O)$, infer that $0=\mathrm{E}_{p}(0)=\mathrm{E}_{p}(u)=$ $\mathrm{E}_{p}(-u)$, and use Proposition 21 as a characterisation for the E-admissible choice functions. On the other hand, $0 \in C_{D_{\mathrm{v}}}^{\mathrm{M}}(O)$ is equivalent to $u,-u \notin \hat{D}$ for some $\hat{D}$ in $\hat{\mathcal{D}}$. But $u \notin \hat{D}$ and $-u \notin \hat{D}$ implies that $-u \in \hat{D}$ and $u \in \hat{D}$ by Proposition 10. a contradiction. So $0 \notin C_{D_{\mathrm{v}}}^{\mathrm{M}}(O)$, whence $C_{\Sigma_{\mathcal{X}}}^{\mathrm{E}} \neq C_{D_{\mathrm{v}}}^{\mathrm{M}}$.

This example shows that $C_{\mathrm{v}}=C_{\overline{\mathcal{D}}} \sqsubset C_{\Sigma_{\mathcal{X}}}^{\mathrm{E}} \sqsubset C_{\hat{\mathcal{D}}}=C_{D_{\mathrm{v}}}^{\mathrm{M}}$.

As remarked by a reviewer, we can interpret the example above in terms of the choice relation associated with a choice function, in the following manner: in the case of a complete preference relation we always have that $\{0\} \triangleleft_{C}\{u,-u\}$ for every option $u$. This is not the case for those induced by sets of strictly desirable options, such as the coherent choice function $C_{p}$ in the example, which therefore cannot be obtained as infima of a family of complete choice relations (as are those given by $M$-admissibility).

In Section 5.7 we will present another example that illustrates the difference between $\mathrm{E}$-admissible and $\mathrm{M}$-admissible choice functions.

There are other coherent sets of desirable options that can be associated with a mass function $p$ and that are intermediate between the strictly desirable and the maximal ones. One example are the so-called lexicographic sets of desirable options, which we have investigated in detail elsewhere [32]. Taking into account Proposition 18, we can also define coherent choice functions by taking the infimum of a family of coherent choice functions determined by such lexicographic sets. Indeed, this provides another example of coherent choice function that admits an axiomatic characterisation in some cases, as reported in [32].

To conclude this section, we want to mention that there are other popular choice rules besides maximality and E-admissibility, such as, amongst others, $\Gamma$-maximin, $\Gamma$-maximax and interval dominance [30]. However, they are not coherent: none of them is guaranteed to satisfy, amongst others, Axiom $\mathrm{C}_{4} \mathrm{~b}$

\section{INDIFFERENCE}

5.1. Indifference and desirability. For sets of desirable gambles, there is a systematic way of modelling indifference $[8,11,20]$. In what follows, we recall how to do this, but formulate everything in terms of the more abstract notion of options, rather than gambles.

In addition to a subject's set of desirable options $D$ - the options he strictly prefers to the zero option - we can also consider the options that he considers to be equivalent to the zero option. We call these options indifferent. A set of indifferent options $I$ is simply a subset of $\mathcal{V}$, but as before with desirable options, we pay special attention to coherent sets of indifferent options.

Definition 13. A set of indifferent options $I$ is called coherent if for all $u, v$ in $\mathcal{V}$ and $\lambda$ in $\mathbb{R}$ :

$\mathrm{I}_{1} .0 \in I$;

$\mathrm{I}_{2}$. if $u \in \mathcal{V}_{>0} \cup \mathcal{V}_{<0}$ then $u \notin I$;

$\mathrm{I}_{3}$. if $u \in I$ then $\lambda u \in I$; 
I 4 . if $u, v \in I$ then $u+v \in I$.

Taken together, Axioms $I_{3}$ and $I_{4}$ are equivalent to imposing that $\operatorname{span}(I)=I$, and due to Axiom $\Pi_{1} I$ is non-empty and therefore a linear subspace of $\mathcal{V}$.

The interaction between indifferent and desirable options is subject to rationality criteria as well: they should be compatible with one another.

Definition 14. Given a set of desirable options $D$ and a coherent set of indifferent options $I$, we call $D$ compatible with $I$ if $D+I \subseteq D$.

The smallest such compatible coherent set of indifferent options is always the trivial one, given by the null space $I=\{0\}$. The idea behind Definition 14 is that adding an indifferent option to a desirable option does not make it non-desirable, or also, that adding a desirable option to an indifferent one makes it desirable.

Since $D \subseteq D+I$ due to Axiom $I_{1}$, compatibility of $D$ and $I$ is equivalent to $D+I=D$. An immediate consequence of compatibility between a coherent set of desirable options $D$ and a coherent set of indifferent options $I$ is that $D \cap I=\varnothing$, meaning that no option can be assessed as desirable—strictly preferred to the zero option—and indifferent—equivalent to the zero option—at the same time.

5.2. Indifference and quotient spaces. In order to introduce indifference for choice functions, we build on a coherent set of indifferent options $I$, as defined in Definition 13 . Two options $u$ and $v$ are considered to be indifferent, to a subject, whenever $v-u$ is indifferent to the zero option, or in other words whenever $v-u \in I$. The underlying idea will be that we identify indifferent options, and choose between equivalence classes of indifferent options, rather than between single options. We formalise this intuition below.

We collect all options that are indifferent to an option $u \in \mathcal{V}$ into the equivalence class

$$
[u]:=\{v \in \mathcal{V}: v-u \in I\}=\{u\}+I .
$$

We also denote $[u]$ as $u / I$. Of course, $[0]=\{0\}+I=I$ is a linear subspace, and the $[u]=$ $\{u\}+I$ are affine subspaces of $\mathcal{V}$. The set of all these equivalence classes is the quotient space

$$
\mathcal{V} / I:=\{[u]: u \in \mathcal{V}\}=\{\{u\}+I: u \in \mathcal{V}\}=\{u / I: u \in \mathcal{V}\}
$$

This quotient space is a vector space under the vector addition, given by

$$
[u]+[v]=\{u\}+I+\{v\}+I=\{u+v\}+I=[u+v] \text { for } u, v \in \mathcal{V},
$$

and the scalar multiplication, given by

$$
\lambda[u]=\lambda(\{u\}+I)=\{\lambda u\}+I=[\lambda u] \text { for } u \in \mathcal{V} \text { and } \lambda \in \mathbb{R} .
$$

$[0]=I$ is the additive identity of $\mathcal{V} / I$.

That we identify indifferent options, and therefore express preferences between equivalence classes of indifferent options, essentially means that we define choice functions on $\mathcal{Q}(\mathcal{V} / I)$. But in order to characterise coherence for such choice functions, we need to introduce a convenient vector ordering on $\mathcal{V} / I$ that is appropriately related to the vector ordering on $\mathcal{V}$; see Section 2.2 For two elements $[u]$ and $[v]$ of $\mathcal{V} / I$, we define

$$
[u] \leq[v] \Leftrightarrow(\exists w \in I) u \leq v+w,
$$

and as usual, the strict variant of the vector ordering on $\mathcal{V} / I$ is characterised by

$$
[u]<[v] \Leftrightarrow([u] \leq[v] \text { and }[u] \neq[v]) .
$$

We begin by showing that this is indeed a vector ordering: 
Proposition 23. The ordering $\leq$ on $\mathcal{V} / I$ is a vector ordering, and $[u]<[v] \Leftrightarrow(\exists w \in I) u<$ $v+w$ for any $u, v$ in $\mathcal{V}$.

We use the notation $O / I:=\{[u]: u \in O\}=\{u / I: u \in O\}$ for the option set of equivalence classes $[u]$ associated with the options $u$ in an option set $O$ in $\mathcal{Q}(\mathcal{V})$. The map $\cdot / I$ is an onto map from $\mathcal{Q}(\mathcal{V})$ to $\mathcal{Q}(\mathcal{V} / I)$ that preserves set inclusion:

$$
\left(\forall O_{1}, O_{2} \in \mathcal{Q}(\mathcal{V})\right)\left(O_{1} \subseteq O_{2} \Rightarrow O_{1} / I \subseteq O_{2} / I\right) .
$$

5.3. Quotient spaces and sets of desirable options. We use this quotient space to prove interesting characterisations of indifference for sets of desirable options.

Proposition 24. A set of desirable options $D \subseteq \mathcal{V}$ is compatible with a coherent set of indifferent options I if and only if there is some (representing) set of desirable options $D^{\prime} \subseteq \mathcal{V} / I$ such that $D=\left\{u:[u] \in D^{\prime}\right\}=\cup D^{\prime}$. Moreover, the representing set of desirable options is unique and given by $D^{\prime}=D / I:=\{[u]: u \in D\}$.

This, together with the definition of compatibility, shows that the correspondence between sets of desirable options on $\mathcal{V}$ and (their representing) sets of desirable options on $\mathcal{V} / I$ is one-to-one and onto. It also preserves coherence.

Proposition 25. Consider any set of desirable options $D \subseteq \mathcal{V}$ that is compatible with a coherent set of indifferent options $I$, and its representing set of desirable options $D / I \subseteq \mathcal{V} / I$. Then $D$ is coherent if and only if $D / I$ is.

5.4. Quotient spaces and choice functions. The discussion above inspires us to combine indifference with choice functions in the following manner: given a coherent set of indifferent options $I$, we say that a choice function is compatible with it when it is determined by its restriction to the quotient space that $I$ induces.

Definition 15. We call a choice function $C$ on $\mathcal{Q}(\mathcal{V})$ compatible with a coherent set of indifferent options $I$ if there is some representing choice function $C^{\prime}$ on $\mathcal{Q}(\mathcal{V} / I)$ such that $C(O)=\left\{u \in O:[u] \in C^{\prime}(O / I)\right\}$ for all $O$ in $\mathcal{Q}(\mathcal{V})$.

This definition can be stated in terms of choice relations as well. We call a choice relation $\triangleleft$ on $\mathcal{Q}(\mathcal{V})$ compatible with $I$ if there is some representing choice relation $\triangleleft^{\prime}$ on $\mathcal{Q}(\mathcal{V} / I)$ such that $O_{1} \triangleleft O_{2} \Leftrightarrow O_{1} / I \triangleleft^{\prime} O_{2} / I$ for all $O_{1}$ and $O_{2}$ in $\mathcal{Q}(\mathcal{V})$.

Proposition 26. Consider any choice function $C$ that is compatible with I, then $\triangleleft_{C}$ is compatible with I as well. Conversely, consider any choice relation $\triangleleft$ that is compatible with I, then $C_{\triangleleft}$ is compatible with I as well.

Definition 15 allows for characterisations that are similar to the ones for desirability in Propositions 24 and 25. If a choice function on $\mathcal{Q}(\mathcal{V})$ is compatible with $I$ then the representing choice function on $\mathcal{Q}(\mathcal{V} / I)$ is necessarily unique, and we denote it by $C / I$ :

Proposition 27. For any choice function $C$ on $\mathcal{Q}(\mathcal{V})$ that is compatible with some coherent set of indifferent options I, the unique representing choice function $C / I$ on $\mathcal{Q}(\mathcal{V} / I)$ is given by $C / I(O / I):=C(O) / I$ for all $O$ in $\mathcal{Q}(\mathcal{V})$. Hence also

$$
C(O)=O \cap(\bigcup C / I(O / I)) \text { for all } O \text { in } \mathcal{Q}(\mathcal{V}) \text {. }
$$

This, together with the definition of compatibility, shows that the correspondence between choice functions on $\mathcal{Q}(\mathcal{V})$ and (their representing) choice functions on $\mathcal{Q}(\mathcal{V} / I)$ is one-to-one and onto. It also preserves coherence: 
Proposition 28. Consider any choice function $C$ on $\mathcal{Q}(\mathcal{V})$ that is compatible with a coherent set of indifferent options I, and its representing choice function $C / I$ on $\mathcal{Q}(\mathcal{V} / I)$. Then $C$ is coherent if and only if $C / I$ is.

To conclude this general discussion of indifference for choice functions, we mention that it is closed under arbitrary infima, which enables conservative inference under indifference: we can consider the least informative choice function that is compatible with some assessments and is still compatible with a given coherent set of indifferent options.

Proposition 29. Consider any coherent set of indifferent options I, and any non-empty collection of coherent choice functions $\left\{C_{i}: i \in \mathcal{I}\right\}$ that are compatible with $I$, then its coherent infimum $\inf \left\{C_{i}: i \in \mathcal{I}\right\}$ is compatible with I as well, and $C / I=\inf \left\{C_{i} / I: i \in \mathcal{I}\right\}$.

In particular, the least informative coherent choice function $C$ that is compatible with a coherent set of indifferent options $I$ corresponds to the case where the associated choice function $C / I$ on the quotient space $\mathcal{Q}(\mathcal{V} / I)$ is the vacuous one $C_{\mathrm{v}}$, meaning that

$$
C(O)=\left\{u \in O:[u] \in C_{\mathrm{v}}(O / I)\right\}=\{u \in O:(\forall v \in O)[u] \nless[v]\} \text { for all } O \text { in } \mathcal{Q}(\mathcal{V}) .
$$

5.5. The relation with other definitions of indifference. Seidenfeld [24] has a rather different approach to combining a notion of indifference with choice functions. After making the necessary translation from horse lotteries to gambles, it can be summarised as follows. Rather than, as we have done above, starting out with a notion of indifference and then looking at which choice functions are compatible with it, he starts from a given choice function $C$, and associates a binary relation $\approx_{C}$ on $\mathcal{V}$ with it as follows:

$$
u \approx_{C} v \Leftrightarrow(\forall O \in \mathcal{Q})(\{u, v\} \subseteq O \Rightarrow(u \in C(O) \Leftrightarrow v \in C(O))) \text { for all } u, v \text { in } \mathcal{V} .
$$

The idea behind this definition is that two options are considered to be related if both options are either chosen or rejected, whenever both are available. This relation has the following interesting properties, which are instrumental in elucidating the relationship between Seidenfeld's approach and ours:

Proposition 30. Let $C$ be a coherent choice function. Then $\approx_{C}$ is an equivalence relation that furthermore satisfies

(i) $u \approx_{C} v \Rightarrow \lambda u \approx C \lambda v$;

(ii) $u \approx_{C} v \Rightarrow u+w \approx_{C} v+w$;

for all $u, v, w \in \mathcal{V}$ and all $\lambda \in \mathbb{R}$. As a consequence, the set $I_{C}:=\left\{u \in \mathcal{V}: u \approx_{C} 0\right\}$ is a coherent set of indifferent options.

Indeed, the coherent set of indifferent options $I_{C}$ turns out to be the largest that $C$ is compatible with:

Proposition 31. Consider a coherent set of indifferent options I and a coherent choice function $C$ on $\mathcal{Q}(\mathcal{V})$. Then $C$ is compatible with I if and only if I is a linear subspace of $I_{C}$.

It follows that if $C$ is compatible with $I$, then $C$ is automatically also compatible with any subspace of $I$, and that the largest linear subspace that $C$ is compatible with, is $I_{C}$. Also, this proposition shows that the smallest set of indifferent option that is compatible with $C$, is given by $I=\{0\}$, and that set of indifferent options is compatible with any coherent choice function. Seidenfeld's approach starts from a choice function, and identifies the coarsest equivalence — or indifference — relation that is compatible with it. Though we have seen that it is related, our approach, because it starts out with an indifference relation, goes the other way around, is more constructive, and is better suited for studying which choice functions are compatible with a given indifference relation, and therefore also for 
performing conservative inference for choice functions under indifference. We will come back to this idea in Sections 5.7 and 5.8

Proposition 31] also shows that our approach is closely connected to Bradley]s [4, Section 2], which he has linked to the notion of sequential coherence: for him, for a choice function to 'satisfy' -in our words: to 'be compatible with' - an equivalence relation, it must be finer than Seidenfeld's $\approx_{C}$.

Finally, note that a relation analogous to Equation 15 can be established for coherent sets of desirable options $D$ : we simply define $u \approx D \nu \Leftrightarrow u \approx C_{D} v$ for all $u$ and $v$ in $\mathcal{V}$. Then

$$
\begin{aligned}
u \approx_{D} v & \Leftrightarrow(\forall O \in \mathcal{Q})\left(\{u, v\} \subseteq O \Rightarrow\left(0 \in C_{D}(O-\{u\}) \Leftrightarrow 0 \in C_{D}(O-\{v\})\right)\right) \\
& \Leftrightarrow(\forall O \in \mathcal{Q})(\{u, v\} \subseteq O \Rightarrow(O-\{u\} \cap D \neq \varnothing \Leftrightarrow O-\{v\} \cap D \neq \varnothing)) \text { for all } u, v \text { in } \mathcal{V},
\end{aligned}
$$

using Axiom $\mathrm{C}_{4} \mathrm{~b}$ for the first equivalence and Equation 8 for the second one. Use Lemma 32 to infer that

$$
u \approx D v \Leftrightarrow D+\{u\}=D+\{v\} \text { for all } u, v \text { in } \mathcal{V} .
$$

Lemma 32. Consider any coherent set of desirable options $D$. Then

$$
(\forall O \in \mathcal{Q})(\{u, v\} \subseteq O \Rightarrow(O-\{u\} \cap D \neq \varnothing \Leftrightarrow O-\{v\} \cap D \neq \varnothing)) \Leftrightarrow D+\{u\}=D+\{v\}
$$

for all $u$ and $v$ in $\mathcal{V}$.

5.6. The relation with desirability. There is an interesting relationship between the coherent choice functions and the coherent sets of desirable options that are compatible with a fixed coherent set of indifferent options.

Proposition 33. Consider any coherent set of indifferent options I.

(i) If $C$ is any coherent choice function compatible with I, then the corresponding coherent set of desirable options $D_{C}$ is also compatible with $I$, and $D_{C} / I=D_{C / I}$.

(ii) If $D$ is any coherent set of desirable options compatible with $I$, then the corresponding coherent choice function $C_{D}$ is also compatible with $I$, and $C_{D} / I=C_{D / I}$.

5.7. Example: fair coins. To exhibit the power and simplicity of our definition of indifference, we reconsider the finite possibility space $\mathcal{X}:=\{\mathrm{H}, \mathrm{T}\}$ of Example 5, where the vector space $\mathcal{V}$ is again the two-dimensional vector space $\mathbb{R}^{\mathcal{X}}$ of real-valued functions on $\mathcal{X}$, or gambles, and the vector ordering $\leq$ is the usual pointwise ordering of gambles.

We want to express indifference between heads and tails, or in other words between $\mathbb{I}_{\mathrm{H}}$ and $\mathbb{I}_{\mathrm{T}}$, where $\mathbb{I}_{\mathrm{H}}:=(1,0)$ and $\mathbb{I}_{\mathrm{T}}:=(0,1)$. This means that $\mathbb{I}_{\mathrm{H}}-\mathbb{I}_{\mathrm{T}}$ is considered equivalent to the zero gamble, so the linear space of all gambles that are equivalent to zero- - or in other words, the set of indifferent gambles (or options) - is then given by

$$
I=\left\{\lambda\left(\mathbb{I}_{\mathrm{H}}-\mathbb{I}_{\mathrm{T}}\right): \lambda \in \mathbb{R}\right\}=\left\{u \in \mathbb{R}^{\mathcal{X}}: \mathrm{E}_{p}(u)=0\right\},
$$

where $\mathrm{E}_{p}$ is the expectation associated with the uniform mass function $p=(1 / 2,1 / 2)$ on $\{\mathrm{H}, \mathrm{T}\}$, associated with a fair coin: $\mathrm{E}_{p}(u):=\frac{1}{2}\left[u_{\mathrm{H}}+u_{\mathrm{T}}\right]$. So, for any option $u$ in $\mathbb{R}^{\mathcal{X}}$-any real-valued function on $\mathcal{X}$ :

$$
[u]=\{u\}+I=\left\{v \in \mathbb{R}^{\mathcal{X}}: \mathrm{E}_{p}(v)=\mathrm{E}_{p}(u)\right\},
$$

which tells us that the equivalence class $[u]$ can be characterised by the common uniform expectation $\mathrm{E}_{p}(u)$ of its elements. Therefore, $\mathbb{R}^{\mathcal{X}} / I$ has unit dimension, and we can identify it with the real line $\mathbb{R}$. The vector ordering between equivalence classes is given by, using Equation 13:

$$
[u] \leq[v] \Leftrightarrow(\exists \lambda \in \mathbb{R}) u \leq v+\lambda\left(\mathbb{I}_{\mathrm{H}}-\mathbb{I}_{\mathrm{T}}\right)
$$




$$
\begin{aligned}
& \Leftrightarrow(\exists \lambda \in \mathbb{R})\left(u_{\mathrm{H}} \leq v_{\mathrm{H}}+\lambda \text { and } u_{\mathrm{T}} \leq v_{\mathrm{T}}-\lambda\right) \\
& \Leftrightarrow(\exists \lambda \in \mathbb{R}) u_{\mathrm{H}}-v_{\mathrm{H}} \leq \lambda \leq-u_{\mathrm{T}}+v_{\mathrm{T}} \\
& \Leftrightarrow u_{\mathrm{H}}-v_{\mathrm{H}} \leq-u_{\mathrm{T}}+v_{\mathrm{T}} \Leftrightarrow \mathrm{E}_{p}(u) \leq \mathrm{E}_{p}(v),
\end{aligned}
$$

and similarly $[u]<[v] \Leftrightarrow \mathrm{E}_{p}(u)<\mathrm{E}_{p}(v)$ for all $u, v$ in $\mathbb{R}^{\mathcal{X}}$. Hence, the strict vector ordering $<$ on $\mathbb{R}^{\mathcal{X}} / I$ is total, so we infer from the argumentation in Example 3 that there is only one representing choice function, namely the vacuous one. Therefore, there is only one choice function $C$ on $\mathcal{Q}\left(\mathbb{R}^{\mathcal{X}}\right)$ that is compatible with $I$, namely, the one that has the vacuous choice function $C_{\mathrm{v}}$ on $\mathcal{Q}\left(\mathbb{R}^{\mathcal{X}} / I\right)$ as its representation $C / I$. Recall that for any $O$ in $\mathcal{Q}\left(\mathbb{R}^{\mathcal{X}}\right)$ :

$$
\begin{aligned}
C_{\mathrm{v}}(O / I) & =\{[u]:(\forall[v] \in O / I)[u] \nless[v]\} \\
& =\{[u]:(\forall[v] \in O / I)[v] \leq[u]\} \\
& =\left\{[u]:(\forall[v] \in O / I) \mathrm{E}_{p}(v) \leq \mathrm{E}_{p}(u)\right\},
\end{aligned}
$$

and therefore

$$
C(O):=\left\{u \in O:(\forall v \in O) \mathrm{E}_{p}(v) \leq \mathrm{E}_{p}(u)\right\}=C_{\{p\}}^{\mathrm{E}}(O) .
$$

The indifference assessment between heads and tails leaves us no choice but to use an E-admissible model for a probability mass function, associated with a fair coin.

The choice function $C$ is therefore based on E-admissibility, but is not compatible with $\mathrm{M}$-admissibility. To see this, consider the set of options $O:=\{w, 0,-w\}$ with $w:=(1,-1)$, so $w_{\mathrm{H}}+w_{\mathrm{T}}=0$. Hence $C(O)=O$; but no M-admissible choice function will select 0 in $O$ : observe that $0 \notin C_{\hat{D}}(O)$ for all $\hat{D} \in \mathcal{D}^{\prime}$, because $0 \in C_{\hat{D}}(O)$ would imply that $\{w,-w\} \cap \hat{D}=\varnothing$, contradicting that $\hat{D}$ is a maximal set of desirable options by Proposition 10 .

5.8. Choice functions and symmetry. As another example showing how powerful our approach to dealing with choice and indifference is, we will prove a simple and elegant representation result that tells us how to perform conservative inference with choice functions under a permutation symmetry assessment.

We consider a finite possibility space $\mathcal{X}$, where the vector space $\mathcal{V}$ of options is the finitedimensional vector space $\mathbb{R}^{r \mathcal{X}}$, of $\mathbb{R}^{r}$-valued functions on $\mathcal{X}$, or vector-valued gambles on the outcome of an uncertain variable $X$ in $\mathcal{X}$. The vector ordering $\leq$ is the usual pointwise ordering of such vector-valued gambles 7

We assume there is symmetry lurking behind the uncertain variable $X$, represented by a group $\mathcal{P}$ of permutations of the set of possible outcomes $\mathcal{X}$ - the idea being that a subject assesses that no distinction should be made between an outcome $x$ and its permutations $\pi x$, for $\pi \in \mathcal{P}$ - or in other words, between the variable $X$ and its permutations $\pi X$. If we consider any vector-valued gamble $u(X)$ on the variable $X$, then the subject will therefore be indifferent between the uncertain vector-valued rewards $u(X)$ and $u(\pi X)$. The smallest coheren 8 set of indifferent options $I_{\mathcal{P}}$ that corresponds to this indifference assessment, is therefore given by

$$
I_{\mathcal{P}}:=\operatorname{span}\left(\left\{u-\pi^{t} u: u \in \mathcal{V} \text { and } \pi \in \mathcal{P}\right\}\right)
$$

\footnotetext{
${ }^{7}$ The reason why we work with vector-valued gambles here rather than real-valued ones, is because Zaffalon and Miranda [36] have shown that this leads to an approach to modelling uncertainty that is even more general than the typical imprecise probability approach.

${ }^{8}$ The requirement that $I_{\mathcal{P}} \cap \mathcal{V}_{<0}=\varnothing$-or equivalently $I_{\mathcal{P}} \cap \mathcal{V}_{>0}=\varnothing$-is related to the left amenability of the finite permutation group $\mathcal{P}$ [9], and is easily shown to be satisfied.
} 
where we defined the linear permutation operator $\pi^{t}$ on the linear space of options (vectorvalued gambles) $\mathcal{V}$ by $\pi^{t} u=u \circ \pi$, or in other words

$$
\left(\pi^{t} u\right)(x):=u(\pi x) \text { for all } u \text { in } \mathcal{V}, x \text { in } \mathcal{X} \text { and } \pi \text { in } \mathcal{P} .
$$

Let us, for any $x$ in $\mathcal{X}$, define the permutation invariant atom $[x]_{\mathcal{P}}$ containing $x$ as

$$
[x]_{\mathcal{P}}:=\{\pi x: \pi \in \mathcal{P}\} .
$$

These atoms constitute a partition of $\mathcal{X}$, and we denote the set of all of them by $\mathcal{A}_{\mathcal{P}}:=$ $\left\{[x]_{\mathcal{P}}: x \in \mathcal{X}\right\}$. A vector-valued gamble $u$ is called $\mathcal{P}$-invariant if $\pi^{t} u=u$ for all $\pi$ in $\mathcal{P}$, and it is not hard to see that this is equivalent to $u$ being constant on the invariant atoms. The set of all $\mathcal{P}$-invariant vector-valued gambles is denoted by $\mathcal{V}_{\mathcal{P}}$, and it is a linear subspace of $\mathcal{V}$ that is clearly isomorphic to the linear space of all vector-valued functions on $\mathcal{A}_{\mathcal{P}}$, whose dimension $r\left|\mathcal{A}_{\mathcal{P}}\right|$ is typically much lower than that of $\mathcal{V}$.

A choice function that takes the symmetry assessment into account is-as we have argued —one that is compatible with $I_{\mathcal{P}}$ and all of its subspaces. What we will do now, is to investigate how such compatible choice functions can be represented by choice functions on a typically much lower-dimensional option space: symmetry reduces complexity. Most of the work for this has already been done in Definition 15 and Proposition 27. which indeed states that choice functions compatible with $I_{\mathcal{P}}$ can be represented uniquely by choice functions on the lower-dimensional quotient space $\mathcal{V} / I_{\mathcal{P}}$. The only thing we need to do, is take a closer look at this quotient space and its elements.

Let us, therefore, define the transformation $\operatorname{inv}_{\mathcal{P}}$ on $\mathcal{V}$ as follows:

$$
\operatorname{inv}_{\mathcal{P}} u:=\frac{1}{|\mathcal{P}|} \sum_{\pi \in \mathcal{P}} \pi^{t} u, \text { for all } u \text { in } \mathcal{V} .
$$

It satisfies the following very interesting properties:

Proposition 34. $\operatorname{inv}_{\mathcal{P}}$ is a linear transformation of $\mathcal{V}$, and

(i) $\operatorname{inv}_{\mathcal{P}} \circ \pi^{t}=\operatorname{inv}_{\mathcal{P}}=\pi^{t} \circ \operatorname{inv}_{\mathcal{P}}$ for all $\pi$ in $\mathcal{P}$;

(ii) $\operatorname{inv}_{\mathcal{P}} \circ \operatorname{inv}_{\mathcal{P}}=\operatorname{inv}_{\mathcal{P}}$;

(iii) $\operatorname{kern}\left(\operatorname{inv}_{\mathcal{P}}\right)=I_{\mathcal{P}}$;

(iv) $\operatorname{rng}\left(\operatorname{inv}_{\mathcal{P}}\right)=\mathcal{V}_{\mathcal{P}}$.

Moreover, for any $u, v$ in $\mathcal{V}$, we have that $v \in u / I_{\mathcal{P}} \Leftrightarrow \operatorname{inv}_{\mathcal{P}} v=\operatorname{inv}_{\mathcal{P}} u$,

The various statements in this proposition tell us that $\operatorname{inv}_{\mathcal{P}}$ is a linear projection operator that maps any vector-valued gamble $u$ to the corresponding uniquely $\mathcal{P}$-invariant member $\operatorname{inv}_{\mathcal{P}} u$ of the equivalence class $u / I_{\mathcal{P}}$, which is essentially a vector-valued gamble on $\mathcal{A}_{\mathcal{P}}$.

By Proposition 27, every coherent choice function $C$ on $\mathcal{V}$ that is compatible with $I_{\mathcal{P}}$ therefore has a unique representing coherent choice function $C_{\mathcal{P}}$ on the typically much lower-dimensional linear space of all vector-valued gambles on $\mathcal{A}_{\mathcal{P}}$, with

$$
C(O)=\left\{u \in O: \operatorname{inv}_{\mathcal{P}} u \in C_{\mathcal{P}}\left(\operatorname{inv}_{\mathcal{P}} O\right)\right\} .
$$

\section{COnClusions}

In this paper, we have shown how the theory of choice functions may be used, with a choice of axioms to model decision problems where the choices are not uniquely determined by the pairwise comparisons, and where models of sets of desirable gambles therefore come up short.

We have made our formalisation in terms of sets of options, because this more general structure has allowed us to make a connection with modelling indifference. Nevertheless, our models remain applicable in particular to choices on sets of gambles, and thus encompass 
much of the behavioural theory of imprecise probabilities as a particular case. A deeper study of the definition in terms of horse lotteries, which is the model considered by Seidenfeld [24], is one of our future lines of research. We have established that choice functions can be equivalent formulated as choice relations, that is, binary relations in terms of sets of options. This formulation adds intuition to some of the results in the paper.

We have seen that three important types of decision models can be subsumed into our theory of choice functions: one, as we have said, is that of sets of desirable options, which give rise to the choice functions defined via maximality; the others are those based on a family of sets of probability measures or, closely related, a family of maximal sets of desirable gambles. We have named these two types of models E-admissible and Madmissible choice functions, and we have shown that there are subtle differences between them. Related to this, one future line of research would be the study of maximal coherent choice functions, which are those that are not above any other choice function. As suggested by one reviewer, it may be useful here to use the equivalent formulation in terms of choice relations and to use the notion of consequence operator [6].

One of the advantages of sets of desirable options over sets of probability measures is that they are more suited to conditioning when sets of (lower) probability zero are involved, as discussed for instance by Quaeghebeur [19]. We intend to investigate in detail in future work how conditioning for choice functions should be defined.

Finally, we have given an axiomatisation of sets of indifferent options and their compatibility with choice functions, and have shown how our definition is related to other approaches proposed in the literature. The investigation of this interesting connection under our framework is another open avenue for future research.

\section{ACKNOWLEDGMENTS}

Gert de Cooman's research was partly funded through project number 3 G012512 of the Research Foundation Flanders (FWO). Erik Quaeghebeur's contribution was partly funded through the Safe Statistics project financed by the Netherlands Organisation for Scientific Research (NWO). Enrique Miranda acknowledges financial support by project TIN201459543-P. The authors would like to express their gratitude to anonymous reviewers of both this paper and an earlier conference version for their helpful comments and suggestions, and to Jasper De Bock for the undominated background noise and stimulating discussion.

\section{Appendix A. PRoOfS}

Proof of Proposition 1. We have to check that $\triangleleft_{C}$ satisfies the two conditions of Definition 2 For (i), consider any $O, O_{1}$ and $O_{2}$ in $\mathcal{Q}$ such that $O_{2} \backslash O_{1} \subseteq O \subseteq O_{1} \cup O_{2}$. Then $O_{1} \cup O=$ $O_{1} \cup O_{2}$, which sets off the following cascade of equivalences:

$$
\begin{aligned}
O_{1} \triangleleft_{C} O & \Leftrightarrow C\left(O_{1} \cup O\right) \subseteq O \backslash O_{1} \\
& \Leftrightarrow C\left(O_{1} \cup O_{2}\right) \subseteq O \backslash O_{1} \\
& \Leftrightarrow C\left(O_{1} \cup O_{2}\right) \subseteq\left(O_{1} \cup O_{2}\right) \cap\left(O \backslash O_{1}\right) \quad \text { because } C\left(O_{1} \cup O_{2}\right) \subseteq O_{1} \cup O_{2} \\
& \Leftrightarrow C\left(O_{1} \cup O_{2}\right) \subseteq O_{2} \backslash O_{1} \Leftrightarrow O_{1} \triangleleft_{C} O_{2},
\end{aligned}
$$

where the penultimate equivalence follows from the equalities: $\left(O_{1} \cup O_{2}\right) \cap\left(O \backslash O_{1}\right)=$ $O_{2} \cap O \cap O_{1}^{c}=O \cap\left(O_{2} \backslash O_{1}\right)=O_{2} \backslash O_{1}$, because by assumption $O \supseteq O_{2} \backslash O_{1}$. For (ii) consider any $O, O_{1}$ and $O_{2}$ in $\mathcal{Q}$ such that $O_{1} \cup O_{2} \subseteq O$. Then $O_{1} \cup O=O_{2} \cup O=O$, which sets off the following cascade of equivalences:

$$
O_{1} \triangleleft_{C} O \text { and } O_{2} \triangleleft_{C} O \Leftrightarrow C\left(O_{1} \cup O\right) \subseteq O \backslash O_{1} \text { and } C\left(O_{2} \cup O\right) \subseteq O \backslash O_{2}
$$




$$
\begin{aligned}
& \Leftrightarrow C(O) \subseteq O \backslash O_{1} \text { and } C(O) \subseteq O \backslash O_{2} \\
& \Leftrightarrow C(O) \subseteq O \backslash\left(O_{1} \cup O_{2}\right) \Leftrightarrow O_{1} \cup O_{2} \triangleleft_{C} O .
\end{aligned}
$$

Proof of Proposition 2. For the first statement, consider any choice function $C$, and any $O$ in $\mathcal{Q}$, and infer that

$$
\begin{aligned}
C_{\triangleleft_{C}}(O)=\bigcap\left\{O^{\prime} \subseteq O: O \backslash O^{\prime} \triangleleft_{C} O\right\} & =\bigcap\left\{O^{\prime} \subseteq O: C\left(\left(O \backslash O^{\prime}\right) \cup O\right) \subseteq O \backslash\left(O \backslash O^{\prime}\right)\right\} \\
& =\bigcap\left\{O^{\prime} \subseteq O: C(O) \subseteq O^{\prime}\right\}=C(O),
\end{aligned}
$$

using the fact that $C(O) \subseteq O$.

For the second statement, consider any choice relation $\triangleleft$, and any $O_{1}$ and $O_{2}$ in $\mathcal{Q}$, and infer that

$$
\begin{aligned}
O_{1} \triangleleft C_{\triangleleft} O_{2} & \Leftrightarrow O_{1} \cap C_{\triangleleft}\left(O_{1} \cup O_{2}\right)=\varnothing \\
& \Leftrightarrow O_{1} \cap\left(O_{1} \cup O_{2} \backslash \bigcup\left\{O^{\prime} \subseteq O_{1} \cup O_{2}: O^{\prime} \triangleleft O_{1} \cup O_{2}\right\}\right)=\varnothing \\
& \Leftrightarrow O_{1} \subseteq \bigcup\left\{O^{\prime} \subseteq O_{1} \cup O_{2}: O^{\prime} \triangleleft O_{1} \cup O_{2}\right\} .
\end{aligned}
$$

Call $O_{3}:=\bigcup\left\{O^{\prime} \subseteq O_{1} \cup O_{2}: O^{\prime} \triangleleft O_{1} \cup O_{2}\right\}$. Since $O_{1} \cup O_{2}$ is finite, and therefore $O_{3}$ is a finite union, we infer from Definition 2[(ii) that $O_{3} \triangleleft O_{1} \cup O_{2}$. If $O_{1} \subseteq O_{3}$ then Definition2/(ii) implies that then $O_{1} \triangleleft O_{1} \cup O_{2}$. Conversely, if $O_{1} \triangleleft O_{1} \cup O_{2}$, then $O_{1} \subseteq O_{3}$ by the definition of $O_{3}$, so $O_{1} \triangleleft C_{\triangleleft} O_{2}$ is equivalent to $O_{1} \triangleleft O_{1} \cup O_{2}$, which is in turn equivalent to $O_{1} \triangleleft O_{2}$ by Definition 2|[i).

For the third statement, assume first that $\triangleleft_{C}=\triangleleft$. Then the corresponding choice functions are equal: $C_{\triangleleft_{C}}=C_{\triangleleft}$. But we have just shown that $C_{\triangleleft_{C}}=C$, whence indeed $C=C_{\triangleleft}$. Conversely, assume that $C_{\triangleleft}=C$. Then the corresponding choice relations are equal: $\triangleleft_{C_{\triangleleft}}=\triangleleft_{C}$. But we have just shown that $\triangleleft_{C_{\triangleleft}}=\triangleleft$, whence indeed $\triangleleft=\triangleleft_{C}$.

Lemma 35. $\tilde{O} \cap C(O)=\varnothing$ is equivalent to $C(O) \subseteq O \backslash \tilde{O}$ for any $C$ in $\mathcal{C}$ and $O, \tilde{O}$ in $\mathcal{Q}$.

Proof. Since for any choice function it holds that $C(O) \subseteq O$, the inclusion $C(O) \subseteq O \backslash \tilde{O}=$ $O \cap \tilde{O}^{c}$ is equivalent to $C(O) \subseteq \tilde{O}^{c}$, that is, to $C(O) \cap \tilde{O}=\varnothing$.

Proof of Axiom $\mathrm{C}_{3} \mathrm{a} \Leftrightarrow$ Equation (3), and of Axiom $\mathrm{C}_{3} \mathrm{~b} \Leftrightarrow$ Equation (4). That Axiom $\mathrm{C}_{3} \mathrm{a}$ and Equation (3) are equivalent follows immediately from Lemma 35

Let us establish the equivalence between Axiom $\mathrm{C}_{3} \mathrm{~b}$ and Equation (4). For necessity, consider any $O, O_{1}, O_{2}$ in $\mathcal{Q}$ such that $O \subseteq O_{1}$ and $O_{1} \cap C\left(O_{2}\right)=\varnothing$, or equivalently, $C\left(O_{2}\right) \subseteq$ $O_{2} \backslash O_{1}$, by Lemma 35 Let $\tilde{O}:=O \cap O_{2}, \tilde{O}_{1}:=O_{2} \backslash O_{1}$ and $\tilde{O}_{2}:=O_{2}$, then $C\left(\tilde{O}_{2}\right) \subseteq \tilde{O}_{1}$, and $\tilde{O}_{2} \backslash \tilde{O}_{1}=O_{2} \backslash\left(O_{2} \backslash O_{1}\right)=O_{1} \cap O_{2}$, whence $\tilde{O}=O \cap O_{2} \subseteq O_{1} \cap O_{2}=\tilde{O}_{2} \backslash \tilde{O}_{1}$. Due to Axiom $\mathrm{C}_{3} \mathrm{~b}$ then $C\left(\tilde{O}_{2} \backslash \tilde{O}\right) \subseteq \tilde{O}_{1}$, whence $C\left(O_{2} \backslash O\right) \subseteq O_{2} \backslash O_{1}$. By Definition 1, we have that $C\left(\mathrm{O}_{2} \backslash \mathrm{O}\right) \subseteq \mathrm{O}_{2} \backslash \mathrm{O}$, and hence $C\left(\mathrm{O}_{2} \backslash \mathrm{O}\right) \subseteq\left(\mathrm{O}_{2} \backslash \mathrm{O}\right) \backslash \mathrm{O}_{1}$. Then indeed $O_{1} \cap$ $\mathrm{C}\left(\mathrm{O}_{2} \backslash \mathrm{O}\right)=\varnothing$, because of Lemma 35 . For sufficiency, consider any $O, O_{1}, O_{2}$ in $\mathcal{Q}$ such that $O \subseteq O_{2} \backslash O_{1}$ and $C\left(O_{2}\right) \subseteq O_{1}$, implying that $\left(O_{2} \backslash O_{1}\right) \cap C\left(O_{2}\right)=\varnothing$. Let $\tilde{O}:=O, \tilde{O}_{1}:=O_{2} \backslash O_{1}$ and $\tilde{O}_{2}:=O_{2}$, then $\tilde{O} \subseteq \tilde{O}_{1}$ and $\tilde{O}_{1} \cap C\left(\tilde{O}_{2}\right)=\varnothing$. Due to Equation (4), then $\tilde{O}_{1} \cap C\left(\tilde{O}_{2} \backslash\right.$ $\tilde{O})=\varnothing$, or equivalently, $C\left(\tilde{O}_{2} \backslash \tilde{O}\right) \subseteq\left(\tilde{O}_{2} \backslash \tilde{O}\right) \backslash \tilde{O}_{1}$, because $C\left(\tilde{O}_{2} \backslash \tilde{O}\right) \subseteq \tilde{O}_{2} \backslash \tilde{O}$. Then $C\left(O_{2} \backslash O\right) \subseteq\left(O_{2} \backslash O\right) \backslash\left(O_{2} \backslash O_{1}\right)$. But $\left(O_{2} \backslash O\right) \backslash\left(O_{2} \backslash O_{1}\right)=O_{2} \cap O_{1}$, implying that indeed $C\left(O_{2} \backslash O\right) \subseteq O_{2} \cap O_{1} \subseteq O_{1}$.

Proof of Proposition 3 For (i) we consider any $O$ in $\mathcal{Q}, \lambda$ in $\mathbb{R}_{>0}$ and $u$ in $\mathcal{V}$, and prove that $\lambda C(O)=C(\lambda O)$ and $C(O)+\{u\}=C(O+\{u\})$. That $\lambda C(O) \subseteq C(\lambda O)$ follows at once from Axiom $\mathrm{C}_{4} \mathrm{a}$. For the converse inclusion, let $O^{\prime}:=\lambda O$, then it follows from Axiom $\mathrm{C}_{4}$ a that $\frac{1}{\lambda} C\left(O^{\prime}\right) \subseteq C\left(\frac{1}{\lambda} O^{\prime}\right)$, which is equivalent to $\frac{1}{\lambda} C(\lambda O) \subseteq C(O)$. Next, that $C(O)+\{u\} \subseteq C(O+\{u\})$ follows directly from Axiom $\mathrm{C}_{4} \mathrm{~b}$. For the converse inclusion, let 
$O^{\prime}:=O+\{u\}$, then it follows from Axiom $\mathrm{C}_{4} \mathrm{~b}$ that $C\left(O^{\prime}\right)+\{-u\} \subseteq C\left(O^{\prime}+\{-u\}\right)$, which is equivalent to $C(O+\{u\}) \subseteq C(O)+\{u\}$.

For (ii), fix any $u_{1}$ and $u_{2}$ in $\mathcal{V}$ such that $u_{1} \leq u_{2}$, any $O$ in $\mathcal{Q}$ and any $v$ in $O \backslash\left\{u_{1}, u_{2}\right\}$. The proof is trivial if $u_{1}=u_{2}$, so assume that $u_{1}<u_{2}$, whence $u_{1} \notin C\left(\left\{u_{1}, u_{2}\right\}\right)$ by Axiom $\mathrm{C}_{2}$ To prove (ii)a assume that $u_{2} \in O$ and $v \notin C\left(O \cup\left\{u_{1}\right\}\right)$, then by version (3) [with $\tilde{O}_{1}:=\left\{u_{1}\right\}$, $\tilde{O}_{2}:=\left\{u_{1}, u_{2}\right\}$ and $\left.\tilde{O}:=O \cup\left\{u_{1}\right\}\right]$ of Axiom $\mathrm{C}_{3}$ a we conclude that $u_{1} \notin C\left(O \cup\left\{u_{1}\right\}\right)$ and therefore $\left\{u_{1}, v\right\} \cap C\left(O \cup\left\{u_{1}\right\}\right)=\varnothing$. Version (4) [with $\tilde{O}_{1}:=\left\{u_{1}, v\right\}, \tilde{O}:=\left\{u_{1}\right\}$ and $\tilde{O}_{2}:=$ $O \cup\left\{u_{1}\right\}$ ] of Axiom $\mathrm{C}_{3} \mathrm{~b}$ then implies that $v \notin C\left(O \backslash\left\{u_{1}\right\}\right)$. Version (3) [with $\tilde{O}_{1}:=\{v\}$, $\tilde{O}_{2}:=O \backslash\left\{u_{1}\right\}$ and $O:=O$ ] of Axiom $\mathrm{C}_{3} \mathrm{a}$ then implies that indeed $v \notin C(O)$. For (ii)b. assume that $u_{1} \in O$ and $v \notin C(O)$, then by version (3) [with $\tilde{O}_{1}:=\left\{u_{1}\right\}$ for the first assumption and $\tilde{O}_{1}:=\{v\}$ for the second one, $\tilde{O}_{2}:=O$ and $\left.\tilde{O}:=\left\{u_{2}\right\} \cup O\right]$ of Axiom $\mathrm{C}_{3} \mathrm{a}$, the first assumption implies that $u_{1} \notin C\left(\left\{u_{2}\right\} \cup O\right)$, and the second assumption implies similarly that $v \notin C\left(\left\{u_{2}\right\} \cup O\right)$, whence $\left\{u_{1}, v\right\} \cap C\left(\left\{u_{2}\right\} \cup O\right)=\varnothing$. By version (4) [with $\tilde{O}_{1}:=\left\{u_{1}, v\right\}$, $\tilde{O}_{2}:=\left\{u_{2}\right\} \cup O$ and $\left.\tilde{O}:=\left\{u_{1}\right\}\right]$ of Axiom $\mathrm{C}_{3} \mathrm{~b}$ this implies that indeed $v \notin C\left(\left\{u_{2}\right\} \cup O \backslash\left\{u_{1}\right\}\right)$.

For (iii), consider any $O, O^{\prime}$ in $\mathcal{Q}$ such that $C(O) \subseteq O^{\prime} \subseteq O$. Let $\tilde{O}_{1}:=C(O), \tilde{O}_{2}:=O$ and $\tilde{O}:=O \backslash O^{\prime}$, and apply Axiom $\mathrm{C}_{3}$ b [we have $C\left(\tilde{O}_{2}\right)=C(O)=\tilde{O}_{1}$, so $C\left(\tilde{O}_{2}\right) \subseteq \tilde{O}_{1}$, and also $\left.\tilde{O}=\tilde{O} \backslash O^{\prime} \subseteq \tilde{O}_{2} \backslash C(O)=\tilde{O}_{2} \backslash \tilde{O}_{1}\right]$ to find that $C\left(\tilde{O}_{2} \backslash \tilde{O}\right) \subseteq \tilde{O}_{1}$, or equivalently, $C\left(O^{\prime}\right) \subseteq C(O)$. Conversely, consider any $u \in C(O)$ and assume ex absurdo that $u \notin C\left(O^{\prime}\right)$, then we infer from version (3) [with $\tilde{O}_{1}:=\{u\}, \tilde{O}_{2}:=O^{\prime}$ and $\tilde{O}:=O$ ] of Axiom $\mathrm{C}_{3}$ a that $u \notin C(O)$, a contradiction.

(iv) is an immediate consequence of (iii) [with $\left.O^{\prime}=C(O)\right]$.

Proof of Proposition 4 For (i), irreflexivity is Axiom $S_{1}$ For transitivity, consider any $O_{1}$, $O_{2}$ and $O_{3}$ in $\mathcal{Q}$, and assume that $O_{1} \triangleleft O_{2}$ and $O_{2} \triangleleft O_{3}$. Use Axiom $\mathrm{S}_{3}$ a to infer that then $O_{1} \triangleleft O_{1} \cup O_{2} \cup O_{3}$ and $O_{2} \triangleleft O_{1} \cup O_{2} \cup O_{3}$. By Definition 2(ii) this is equivalent to $O_{1} \cup O_{2} \triangleleft O_{1} \cup O_{2} \cup O_{3}$. Now, use Axiom $\mathrm{S}_{3} \mathrm{~b}$ [with $\tilde{O}_{1}:=O_{1} \cup O_{2}, O_{2}:=O_{1} \cup O_{2} \cup O_{3}$ and $\tilde{O}:=O_{2} \backslash\left(O_{1} \cup O_{3}\right) \subseteq \tilde{O}_{1}$; then $\tilde{O}_{1} \backslash \tilde{O}=\left(O_{1} \cup O_{2}\right) \backslash\left(O_{2} \backslash\left(O_{1} \cup O_{3}\right)\right)=O_{1} \cup\left(O_{2} \cap O_{3}\right)$ and $\left.\tilde{O}_{2} \backslash \tilde{O}=\left(O_{1} \cup O_{2} \cup O_{3}\right) \backslash\left(O_{2} \backslash\left(O_{1} \cup O_{3}\right)\right)=O_{1} \cup O_{3}\right]$ to infer that $O_{1} \cup\left(O_{2} \cap O_{3}\right) \triangleleft O_{1} \cup O_{3}$, and therefore $O_{1} \triangleleft O_{1} \cup O_{3}$ using Definition 2[(ii) Then indeed $O_{1} \triangleleft O_{3}$ by Definition 2(i)

For (ii), consider any $O_{1}$ and $O_{2}$ in $\mathcal{Q}$, any $u$ in $\mathcal{V}$ and any $\lambda$ in $\mathbb{R}_{>0}$. For the direct implication, assume that $O_{1} \triangleleft O_{2}$. Then $\lambda O_{1} \triangleleft \lambda O_{2}$ by Axiom $\mathrm{S}_{4}$ a and therefore indeed $\lambda O_{1}+\{u\} \triangleleft \lambda O_{2}+\{u\}$ by Axiom $\mathrm{S}_{4} \mathrm{~b}$. Conversely, for the converse implication, assume that $\lambda O_{1}+\{u\} \triangleleft \lambda O_{2}+\{u\}$. Then $\lambda O_{1} \triangleleft \lambda O_{2}$ by Axiom $\mathrm{S}_{4} \mathrm{~b}$ and therefore indeed $O_{1} \triangleleft O_{2}$ by Axiom $\mathrm{S}_{4} \mathrm{a}$.

Proof of Proposition 5. We first prove the first statement. We show that $\triangleleft_{C}$ satisfies the Axioms $\mathrm{S}_{1} \mathrm{~S}_{4}$

For Axiom $\mathrm{S}_{1}$, consider any $O$ in $\mathcal{Q}$. Since $C(O) \neq \varnothing$ by Axiom $\mathrm{C}_{1}$, we infer that $C(O) \nsubseteq \varnothing=O \backslash O$, whence indeed $O{ }_{C} O$.

For Axiom $\mathbf{S}_{2}$, assume that $u<v$. Then $\{v\}=C(\{u, v\})$ by Axiom $\mathbf{C}_{2}$, so $C(\{u, v\}) \subseteq$ $\{v\} \backslash\{u\}$. Then indeed $\{u\} \triangleleft_{C}\{v\}$.

For Axiom $\mathrm{S}_{3} \mathrm{a}$, assume that $O_{1} \triangleleft_{C} O_{2}$ and $O_{2} \subseteq O$. Then $O_{1} \cap C\left(O_{1} \cup O_{2}\right)=\varnothing$ by Definition 3 . Use version (3) of Axiom $\mathrm{C}_{3}$ a [with $\tilde{O}_{1}:=O_{1}, \tilde{O}_{2}:=O_{1} \cup O_{2}$ and $\tilde{O}:=O_{1} \cup O$; then $\tilde{O}_{1} \subseteq \tilde{O}_{2} \subseteq \tilde{O}$ since $\left.O_{2} \subseteq O\right]$ to infer that then $O_{1} \cap C\left(O_{1} \cup O\right)=\varnothing$, whence indeed $O_{1} \triangleleft_{C} O$.

For Axiom $\mathrm{S}_{3} \mathrm{~b}$, assume that $O_{1} \triangleleft_{C} O_{2}$ and $O \subset O_{1}$. Then $O_{1} \cap C\left(O_{1} \cup O_{2}\right)=\varnothing$ by Definition 3. Use version (4) of Axiom $\mathrm{C}_{3}$ b [with $\tilde{O}_{1}:=O_{1}, \tilde{O}_{2}:=O_{1} \cup O_{2}$ and $\tilde{O}:=O$; then $\left.\tilde{O} \subseteq \tilde{O}_{1}\right]$ to infer that $O_{1} \cap C\left(\left(O_{1} \cup O_{2}\right) \backslash O\right)=\varnothing$, and hence in particular that $\left(O_{1} \backslash\right.$ $O) \cap C\left(\left(O_{1} \cup O_{2}\right) \backslash O\right)=\varnothing$. Then $O_{1} \backslash O \triangleleft_{C}\left(O_{1} \backslash O\right) \cup\left(O_{2} \backslash O\right)$. Use Definition 2 [i) [with 
$\tilde{O}_{1}:=O_{1} \backslash O, \tilde{O}_{2}:=\left(O_{1} \backslash O\right) \cup\left(O_{2} \backslash O\right)$ and $\tilde{O}:=O_{2} \backslash O$; then $\left.\tilde{O}_{2} \backslash \tilde{O}_{1} \subseteq \tilde{O} \subseteq \tilde{O}_{1} \cup \tilde{O}_{2}\right]$ to infer that then indeed $O_{1} \backslash O \triangleleft_{C} O_{2} \backslash O$.

For Axioms $\mathrm{S}_{4} \mathrm{a}$ and $\mathrm{S}_{4} \mathrm{~b}$, assume that $O_{1} \triangleleft_{C} O_{2}$. We need to show that then $\lambda O_{1}+\{u\} \triangleleft_{C}$ $\lambda O_{2}+\{u\}$. To do so, from $O_{1} \triangleleft_{C} O_{2}$ infer that $O_{1} \cap C\left(O_{1} \cup O_{2}\right)=\varnothing$, so $\left(\lambda O_{1}+\{u\}\right) \cap$ $\left(\lambda C\left(O_{1} \cup O_{2}\right)+\{u\}\right)=\varnothing$. Then use Proposition $3(\mathrm{i})$ to infer that $\left(\lambda O_{1}+\{u\}\right) \cap C\left(\lambda\left(O_{1} \cup\right.\right.$ $\left.\left.O_{2}\right)+\{u\}\right)=\varnothing$, whence indeed $\lambda O_{1}+\{u\} \triangleleft_{C} \lambda O_{2}+\{u\}$.

For the second statement, we need to show that $C_{\triangleleft}$ satisfies the Axioms $\mathrm{C}_{1} \mathrm{C}_{4}$

For Axiom $\mathrm{C}_{1}$, consider any $O$ in $\mathcal{Q}$. Then $O \rtimes O$ by Axiom $\mathrm{S}_{1}$, so $\{u\} \pitchfork O$ for some $u$ in $O$, by Definition 2(ii) Then $u \in C_{\triangleleft}(O):=O \backslash \cup\left\{O^{\prime} \subseteq O: O^{\prime} \triangleleft O\right\}$, so $C_{\triangleleft}(O)$ is indeed non-empty.

For Axiom $\mathbf{C}_{2}$, assume that $u<v$. Then $\{u\} \triangleleft\{v\}$ by Axiom $\mathbf{S}_{2}$, so $C_{\triangleleft}(\{u, v\}) \cap\{u\}=\varnothing$. Since $C_{\triangleleft}(\{u, v\})$ is non-empty, as shown above, then necessarily $v \in C_{\triangleleft}(\{u, v\})$, so indeed $\{v\}=C_{\triangleleft}(\{u, v\})$.

For version (3) of Axiom $\mathrm{C}_{3} \mathrm{a}$, assume that $O_{1} \cap C_{\triangleleft}\left(O_{2}\right)=\varnothing$ and $O_{1} \subseteq O_{2} \subseteq O$. Since $O_{1} \subseteq O_{2}$, then $O_{2}=O_{1} \cup O_{2}$, so $O_{1} \cap C_{\triangleleft}\left(O_{1} \cup O_{2}\right)=\varnothing$, whence $O_{1} \triangleleft O_{2}$. Then $O_{1} \triangleleft O$ by Axiom $\mathrm{S}_{3}$ a so $O_{1} \cap C_{\triangleleft}\left(O \cup O_{1}\right)=\varnothing$, and since $O \cup O_{1}=O$, indeed $O_{1} \cap C_{\triangleleft}(O)=\varnothing$.

For version (4) of Axiom $\mathrm{C}_{3} \mathrm{~b}$, assume that $O_{1} \cap C_{\triangleleft}\left(O_{2}\right)=\varnothing$ and $O \subseteq O_{1}$. We need to prove that then $O_{1} \cap C_{\triangleleft}\left(O_{2} \backslash O\right)=\varnothing$. From $O_{1} \cap C_{\triangleleft}\left(O_{2}\right)=\varnothing$, and using that $C_{\triangleleft}\left(O_{2}\right)=$ $O_{2} \backslash \bigcup\left\{O^{\prime} \subseteq O_{2}: O^{\prime} \triangleleft O_{2}\right\}$, infer that $O_{1} \cap O_{2} \subseteq \bigcup\left\{O^{\prime} \subseteq O_{2}: O^{\prime} \triangleleft O_{2}\right\}$, whence $O_{1} \cap O_{2} \triangleleft O_{2}$, taking into account Definition/2](ii) Call $O^{\prime}:=O \cap O_{2}$, then $\left(O_{1} \cap O_{2}\right) \backslash O^{\prime}=\left(O_{1} \cap O_{2}\right) \backslash O$ and $O_{2} \backslash O^{\prime}=O_{2} \backslash O$. Also $O^{\prime} \subseteq O_{1} \cap O_{2}$, so either $O^{\prime}=O_{1} \cap O_{2}$ or $O^{\prime} \subset O_{1} \cap O_{2}$. If $O^{\prime}=$ $O_{1} \cap O_{2}$ then $\left(O_{1} \cap O_{2}\right) \backslash O^{\prime}=\varnothing$ whence $\left(O_{1} \cap O_{2}\right) \backslash O^{\prime} \cap C\left(O_{2} \backslash O^{\prime}\right)=\varnothing$ trivially. But since $C\left(O_{2} \backslash O^{\prime}\right) \subseteq O_{2} \backslash O^{\prime}$, then also $O_{1} \cap C\left(O_{2} \backslash O^{\prime}\right)=\varnothing$ and hence indeed $O_{1} \cap C\left(O_{2} \backslash O\right)=$ $\varnothing$. So assume $O^{\prime} \subset O_{1} \cap O_{2}$. Then Axiom $\mathrm{S}_{3}$ b implies that $\left(O_{1} \cap O_{2}\right) \backslash O^{\prime} \triangleleft O_{2} \backslash O^{\prime}$, or equivalently, replacing $O^{\prime}$ with $O \cap O_{2}$, that $\left(O_{1} \cap O_{2}\right) \backslash\left(O \cap O_{2}\right) \triangleleft O_{2} \backslash\left(O \cap O_{2}\right)$. Infer that $\left(O_{1} \cap O_{2}\right) \backslash\left(O \cap O_{2}\right)=\left(O_{1} \cap O_{2}\right) \backslash O$ and $O_{2} \backslash\left(O \cap O_{2}\right)=O_{2} \backslash O$, and therefore $\left(O_{1} \cap\right.$ $\left.O_{2}\right) \backslash O \triangleleft O_{2} \backslash O$. Then $\left(O_{1} \cap O_{2}\right) \backslash O \cap C_{\triangleleft}\left(O_{2} \backslash O\right)=\varnothing$, and since $C_{\triangleleft}\left(O_{2} \backslash O\right) \subseteq O_{2} \backslash O$, we find that indeed $O_{1} \cap C_{\triangleleft}\left(O_{2} \backslash O\right)=\varnothing$.

For Axioms $\mathrm{C}_{4} \mathrm{a}$ and $\mathrm{C}_{4} \mathrm{~b}$, assume that $O_{1} \subseteq C_{\triangleleft}\left(O_{2}\right)$. We need to show that then $\lambda O_{1}+$ $\{u\} \subseteq C_{\triangleleft}\left(\lambda O_{2}+\{u\}\right)$. To do so, from $O_{1} \subseteq C_{\triangleleft}\left(O_{2}\right)$ infer that $O_{1} \subseteq O_{2}$ and hence $O_{2}=$ $O_{1} \cup O_{2}$, whence $C_{\triangleleft}\left(O_{1} \cup O_{2}\right) \subseteq O_{2} \backslash O_{1}$. Then $O_{1}$ rel $O_{2}$, so $\lambda O_{1}+\{u\} \triangleleft \lambda O_{2}+\{u\}$ by Axioms $\mathrm{S}_{4} \mathrm{a}$ and $\mathrm{S}_{4} \mathrm{~b}$, whence $C_{\triangleleft}\left(\lambda\left(O_{1} \cup O_{2}\right)+\{u\}\right) \subseteq \lambda\left(O_{2} \backslash O_{1}\right)+\{u\}$. Taking into account that $O_{1} \cup O_{2}=O_{2}$, then indeed $\lambda O_{1}+\{u\} \subseteq C_{\triangleleft}\left(\lambda O_{2}+\{u\}\right)$.

Proof of Proposition 7. The map $f$ we are looking for is $f: \mathcal{C} \rightarrow \mathcal{S}: C \mapsto f(C):=\triangleleft_{C}$. Due to Proposition 2, $f$ is an invertible function with inverse $f^{-1}: \mathcal{S} \rightarrow \mathcal{C}: \triangleleft \mapsto f^{-1}(\triangleleft):=C_{\triangleleft}$, and hence $f$ is a bijection between $\mathcal{C}$ and $\mathcal{S}$.

For the first statement, we prove that $(\mathcal{C}$;드 $)$ and $(\mathcal{S} ; \sqsubseteq \mathcal{S})$ are order isomorphic. We first show that $C_{1} \sqsubseteq C_{2} \Rightarrow f\left(C_{1}\right) \sqsubseteq \mathcal{S} f\left(C_{2}\right)$. So consider any two choice functions $C_{1}$ and $C_{2}$ such that $C_{1} \sqsubseteq C_{2}$. Then $C_{1}(O) \supseteq C_{2}(O)$ for all $O$ in $\mathcal{Q}$. Consider any $O_{1}$ and $O_{2}$ in $\mathcal{Q}$ and assume that $O_{1} \triangleleft{ }_{C_{1}} O_{2}$. Then $O_{1} \cap C_{1}\left(O_{1} \cup O_{2}\right)=\varnothing$, and hence also $O_{1} \cap C_{2}\left(O_{1} \cup O_{2}\right)=\varnothing$. But then $O_{1} \triangleleft_{C_{2}} O_{2}$, so indeed $\triangleleft_{C_{1}}{ }_{\mathcal{S}} \triangleleft_{C_{2}}$. To show that $f\left(C_{1}\right) \sqsubseteq \mathcal{S} f\left(C_{2}\right) \Rightarrow C_{1} \sqsubseteq C_{2}$, it suffices to consider any two choice relation $\triangleleft_{1}$ and $\triangleleft_{2}$ such that $\triangleleft_{1} \sqsubseteq \mathcal{S} \triangleleft_{2}$, and prove that $C_{\triangleleft_{1}} \sqsubseteq C_{\triangleleft_{2}}$, because the map $f$ is a bijection. So assume that $\triangleleft_{1} \check{S}_{\mathcal{S}} \triangleleft_{2}$. Then $O_{1} \triangleleft_{1} O_{2} \Rightarrow O_{1} \triangleleft_{2} O_{2}$ for all $O_{1}$ and $O_{2}$ in $\mathcal{Q}$. Consider any $O$ in $\mathcal{Q}$, and any $u$ in $O$ such that $u \notin C_{\triangleleft_{1}}(O)=O \backslash \bigcup\left\{O^{\prime} \subseteq O\right.$ : $\left.O^{\prime} \triangleleft_{1} O\right\}$. Then $u \in \bigcup\left\{O^{\prime} \subseteq O: O^{\prime} \triangleleft_{1} O\right\}$, but since $\triangleleft_{1} \subseteq \triangleleft_{2}$, then also $u \in \bigcup\left\{O^{\prime} \subseteq O: O^{\prime} \triangleleft_{2} O\right\}$. Then $u \notin C_{\triangleleft_{2}}(O)$, so indeed $C_{\triangleleft_{1}} \sqsubseteq C_{\triangleleft_{2}}$. 
For the second statement, Proposition 5 guarantees that the same map is a bijection between $\overline{\mathcal{C}}$ and $\overline{\mathcal{S}}$. Since we have already shown that both $f$ and its inverse $f^{-1}$ preserve the order, this completes the proof.

Proof of Proposition 8 . We show that inf $\mathcal{C}^{\prime}$ satisfies the rationality axioms of Definition 4 $\mathrm{C}_{1}$ Consider any $C$ in $\mathcal{C}^{\prime}$ [always possible since $\mathcal{C}^{\prime} \neq \varnothing$ ] and any $O$ in $\mathcal{Q}$, then $\varnothing \subset C(O) \subseteq$ $\inf \mathcal{C}^{\prime}(O)$.

$\mathrm{C}_{2}$ Note that, for all $C$ in $\mathcal{C}^{\prime}, C(\{u, v\})=\{v\}$ whenever $u<v$, implying that $\inf \mathcal{C}^{\prime}(\{u, v\})=$ $\cup_{C \in \mathcal{C}^{\prime}} C(\{u, v\})=\{v\}$.

$\mathrm{C}_{3}$ a Fix any $O, O_{1}, O_{2}$ in $\mathcal{Q}$ such that $\cup_{C \in \mathcal{C}^{\prime}} C\left(O_{2}\right)=\inf \mathcal{C}^{\prime}\left(O_{2}\right) \subseteq O_{2} \backslash O_{1}$ and $O_{1} \subseteq O_{2} \subseteq O$. This implies that for all $C$ in $\mathcal{C}^{\prime}, C\left(O_{2}\right) \subseteq O_{2} \backslash O_{1}$ and therefore also $C(O) \subseteq O \backslash O_{1}$, because $C$ is coherent. So indeed $\inf \mathcal{C}^{\prime}(O)=\bigcup_{C \in \mathcal{C}^{\prime}} C(O) \subseteq O \backslash O_{1}$.

$\mathrm{C}_{3} \mathrm{~b}$ Fix any $O, O_{1}, O_{2}$ in $\mathcal{Q}$ such that $\inf _{\mathcal{C}^{\prime}}\left(O_{2}\right)=\bigcup_{C \in \mathcal{C}^{\prime}} C\left(O_{2}\right) \subseteq O_{1}$ and $O \subseteq O_{2} \backslash O_{1}$. This means that for all $C$ in $\mathcal{C}^{\prime}, C\left(O_{2}\right) \subseteq O_{1}$, and therefore also $C\left(O_{2} \backslash O\right) \subseteq O_{1}$, because $C$ is coherent. So indeed $\inf \mathcal{C}^{\prime}\left(O_{2} \backslash O\right)=\bigcup_{C \in \mathcal{C}^{\prime}} C\left(O_{2} \backslash O\right) \subseteq O_{1}$.

$\mathrm{C}_{4}$ Consider any $C$ in $\mathcal{C}^{\prime}, \lambda$ in $\mathbb{R}_{>0}, u$ in $\mathcal{V}$ and $O$ in $\mathcal{Q}$, and derive from Proposition 3(i) that $\lambda C(O)+\{u\}=C(\lambda O+\{u\})$. Hence $\lambda \inf \mathcal{C}^{\prime}(O)+\{u\}=\lambda \cup_{C \in \mathcal{C}^{\prime}} C(O)+\{u\}=$ $\cup_{C \in \mathcal{C}^{\prime}}(\lambda C(O)+\{u\})=\cup_{C \in \mathcal{C}^{\prime}} C(\lambda O+\{u\})=\inf \mathcal{C}^{\prime}(\lambda O+\{u\})$, which implies that $\inf \mathcal{C}^{\prime}$ satisfies axioms $\mathrm{C}_{4} \mathrm{a}$ and $\mathrm{C}_{4} \mathrm{~b}$.

Proof of Proposition 9. Consider any set of coherent sets of desirable options $\mathcal{D}^{\prime} \subseteq \overline{\mathcal{D}}$, and its infimum inf $\mathcal{D}^{\prime}=\bigcap \mathcal{D}^{\prime}$, which is of course also a set of desirable options. We show that $\inf \mathcal{D}^{\prime}$ is coherent, meaning that it satisfies the rationality axioms of Definition 7 .

$\mathrm{D}_{1}$ Since $0 \notin D$ for all $D$ in $\mathcal{D}^{\prime}$, also $0 \notin \inf \mathcal{D}^{\prime}$.

$\mathrm{D}_{2}$ Fix any $u$ in $\mathcal{V}_{>0}$, so $u \in D$ for all $D$ in $\mathcal{D}^{\prime}$, implying that indeed $u \in \inf \mathcal{D}^{\prime}$.

$\overline{\mathrm{D}}_{3}$ Consider any $\lambda$ in $\mathbb{R}_{>0}$ and any $u \in \inf \mathcal{D}^{\prime}$, meaning that $u \in D$ for all $D$ in $\mathcal{D}^{\prime}$. Then also $\lambda u \in D$ for all $D$ in $\mathcal{D}^{\prime}$, implying that indeed $\lambda u \in \inf \mathcal{D}^{\prime}$.

$\mathrm{D}_{4}$ Consider any $u, v \in \inf \mathcal{D}^{\prime}$, meaning that $u$ and $v$ belong to all $D$ in $\mathcal{D}^{\prime}$. Hence $u+v \in D$ for all $D$ in $\mathcal{D}^{\prime}$, implying that indeed $u+v \in \inf \mathcal{D}^{\prime}$.

Now, as a convex cone, $\mathcal{V}_{>0}$ satisfies $\mathrm{D}_{3}$ and $\mathrm{D}_{4}$, and by definition it satisfies $\mathrm{D}_{1}$ and $\mathrm{D}_{2}$ So $\mathcal{V}_{>0}$ is coherent, and by Axiom $\mathrm{D}_{2}$ it is included in any other coherent set of desirable options.

Proof of Proposition 10. Let, for ease of notation, $D^{\prime}:=\operatorname{posi}(D \cup\{-u\})$. It is clear that $D^{\prime}$ satisfies Axioms $\mathrm{D}_{2} \mathrm{D}_{4}$, so we only need to prove that $0 \notin D^{\prime}$. Assume ex absurdo that $0 \in D^{\prime}$. Since $0 \notin D$ and $0 \neq u$, there must be $v$ in $D$ and $\lambda$ in $\mathbb{R}_{>0}$ such that $v+\lambda(-u)=0$, implying that $u=\frac{1}{\lambda} v \in D\left[\right.$ Axiom $\mathrm{D}_{3}$, a contradiction.

Next, consider any maximal coherent set of desirable options $D$ and fix any option $u$ in $\mathcal{V} \backslash\{0\}$ such that $-u \notin D$. Assume ex absurdo that also $u \notin D$, then $D^{\prime}:=\operatorname{posi}(D \cup\{-u\}) \supset D$ is a coherent set of desirable options by the first part, contradicting the maximality of $D$.

Conversely, consider any coherent set of desirable options $D$ that satisfies Equation (5), and any coherent set of desirable options $D^{\prime} \supseteq D$. Consider any $u$ in $D^{\prime}$ then clearly $u \neq 0$ [Axiom $\mathrm{D}_{1}$, , so we infer from (5] that $u \in D$ or $-u \in D$. Assume ex absurdo that $-u \in D$, then also $-u \in D^{\prime}$, which together with $u \in D^{\prime}$ implies that $0=u+(-u) \in D^{\prime}$ [Axiom $\left.\mathrm{D}_{4}\right]$, which contradicts the coherence of $D^{\prime}$ [Axiom $\mathrm{D}_{1}$ ]. Hence $u \in D$, which implies that $D^{\prime}=D$, so $D$ is indeed maximal.

Proof of Proposition 11. We have to prove that the set $\{\hat{D} \in \overline{\mathcal{D}}: D \subseteq \hat{D}\}$ has a maximal element. This will follow directly from Zorn's Lemma if we can show that any chain $\mathbb{K}$ in 
this poset has a greatest element. It is a matter of straightforward verification of the axioms to see that $\cup \mathbb{K}$ is a coherent set of desirable options, so $\cup \mathbb{K}$ is this greatest element.

Let us now establish the dual atomicity. By definition, $D \subseteq \cap \hat{\mathcal{D}}_{D}$, so we concentrate on proving that $\cap \hat{\mathcal{D}}_{D} \subseteq D$. Consider any $u$ in $\cap \hat{\mathcal{D}}_{D}$, meaning that $u \in \hat{D}$ for all $\hat{D} \in \hat{\mathcal{D}}_{D}$. Assume ex absurdo that $u \notin D$, then $-u \in \operatorname{posi}(D \cup\{-u\})$, and $\operatorname{posi}(D \cup\{-u\})$ is a coherent set of desirable options by Proposition 10. Consider any maximal coherent set of desirable options $\hat{D}$ that dominates this set [there is such a coherent maximal set by Proposition 11], then also $-u \in \hat{D}$ and therefore $u \notin \hat{D}$ [use Axioms $\mathrm{D}_{4}$ and $\mathrm{D}_{1}$. But since the maximal $\hat{D}$ also dominates $D$, this is a contradiction.

Proof of Proposition 12 We first show that $D_{C}$ is coherent:

$\mathrm{D}_{1}$ Assume ex absurdo that $0 \in D_{C}$, meaning that $0 \notin C(\{0\})$, contradicting the coherence of $C\left[\right.$ Axiom $\left.\mathrm{C}_{1}\right]$.

$\mathrm{D}_{2}$ Consider any option $u$ in $\mathcal{V}_{>0}$, so $0<u$. The coherence of $C$ [Axiom $\left.\mathrm{C}_{2}\right]$ then implies that $\{u\}=C(\{0, u\})$, whence $0 \notin C(\{0, u\})$, implying that indeed $u \in D_{C}$.

$\mathrm{D}_{3}$ Consider any $\lambda$ in $\mathbb{R}_{>0}$, and any $u$ in $D_{C}$, meaning that $0 \notin C(\{0, u\})$. Proposition 3. (i) with $v=0$ then implies that $0=\lambda 0 \notin C(\{0, \lambda u\})$, whence indeed $\lambda u \in D_{C}$.

$\mathrm{D}_{4}$ Consider any $u, v$ in $D$, meaning that $0 \notin C(\{0, u\})$ and $0 \notin C(\{0, v\})$. Proposition 3(i) with $\mu=1$ then implies that $u \notin C(\{u, u+v\})$ and $v \notin C(\{v, u+v\})$, and consequently that $\{0, u, v\} \cap C(\{0, u, v, u+v\})=\varnothing$ [use version (3) of Axiom $\mathrm{C}_{3}$ a three times with $O:=\{0, u, v, u+v\}:$ once with $O_{1}:=\{0\}$ and $O_{2}:=\{0, u\}$, once with $O_{1}:=\{u\}$ and $O_{2}:=\{u, u+v\}$, and once with $O_{1}:=\{v\}$ and $\left.O_{2}:=\{v, u+v\}\right]$. This implies that $0 \notin$ $C(\{0, u+v\})$ by version (4) [with $O_{3}:=\{0, u, v\}, O_{2}:=\{0, u, v, u+v\}$ and $O:=\{u, v\}$ ] of Axiom $\mathrm{C}_{3} \mathrm{~b}$, whence indeed $u+v \in D_{C}$.

We complete the proof by showing that $C$ and $D_{C}$ are compatible: $v \notin C(\{u, v\}) \Leftrightarrow u-v \epsilon$ $D_{C}$ for all $u, v$ in $\mathcal{V}$. For the direct implication, consider any $u, v$ in $\mathcal{V}$ such that $v \notin C(\{u, v\})$. Proposition 3 (i) with $\lambda=1$ then guarantees that $0 \notin C(\{0, u-v\})$, implying that indeed $u-v \in D_{C}$. For the converse implication, consider any $u, v$ in $\mathcal{V}$ such that $u-v \in D_{C}$. Then $0 \notin C(\{0, u-v\})$, implying that indeed $v \notin C(\{u, v\})$, by Proposition 3 (i) with $\lambda=1$.

Proof of Proposition 13 . The proof is structured as follows: we show (a) that $C_{D}$ is compatible with $D$; (b) that $\bar{C}_{D}$ is coherent; and (c) that $C_{D} \sqsubseteq C$ for all $C \in \overline{\mathcal{C}}_{D}$.

(a) First, we show that $C_{D}$ is compatible with $D$ : Consider any $u, v$ in $\mathcal{V}$ then it follows from the definition of $C_{D}$ that indeed

$v \notin C_{D}(\{u, v\}) \Leftrightarrow(\exists w \in\{u, v\}) w-v \in D \Leftrightarrow(u-v \in D$ or $v-v \in D) \Leftrightarrow u-v \in D$,

where the last equivalence follows from $0 \notin D$, because $D$ is coherent [Axiom $\mathrm{D}_{1}$ ].

(b) Next, we show that $C_{D}$ is coherent:

$\mathrm{C}_{1}$ Consider any $O$ in $\mathcal{Q}$. Since $|O|$ is finite and $\triangleleft$ is a strict partial order, we know that there is at least one maximal element $u_{m}$ for $\varangle$, meaning that $(\forall v \in O) u_{m} \nless v$, or equivalently $(\forall v \in O) v-u_{m} \notin D$. Hence $u_{m} \in C_{D}(O)$.

$\mathrm{C}_{2}$ Consider any $u, v$ in $\mathcal{V}$ such that $u<v$. Then $0<v-u$, whence $v-u \in D$ by Axiom $\mathrm{D}_{2}$. So $u \notin C_{D}(\{u, v\})$ by compatibility. Since we already know that $C_{D}(\{u, v\}) \neq \varnothing$, we must therefore have that indeed $\{v\}=C_{D}(\{u, v\})$.

(3) Consider any option sets $O, O_{1}, O_{2}$ in $\mathcal{Q}$ such that $O_{1} \subseteq O_{2} \subseteq O$ and $O_{1} \cap C_{D}\left(O_{2}\right)=$ $\varnothing$, implying that $u \notin C_{D}\left(O_{2}\right)$ for all $u$ in $O_{1}$, or equivalently, that $\left(\forall u \in O_{1}\right)(\exists v \in$ $\left.O_{2}\right) v-u \in D$. Since $O_{2} \subseteq O$, this implies that $\left(\forall u \in O_{1}\right)(\exists v \in O) v-u \in D$, or indeed $O_{1} \cap C_{D}(O)=\varnothing$.

(44) Consider any option sets $O, O_{1}, O_{2}$ in $\mathcal{Q}$ such that $O \subseteq O_{1}$ and $O_{1} \cap C_{D}\left(O_{2}\right)=\varnothing$, implying that $\left(\forall u \in O_{1}\right)\left(\exists v \in O_{2}\right) v-u \in D$. We need to prove that $\left(\forall u \in O_{1}\right)(\exists v \in$ 
$\left.O_{2} \backslash O\right) v-u \in D$. We may assume without loss of generality that $O=\left\{v_{1}, \ldots, v_{n}\right\}$ with $n$ in $\mathbb{N}_{0}$. Consider any $u$ in $O_{1}$, then we know that $u_{1}-u \in D$ for some $u_{1}$ in $O_{2}$. Then either $u_{1} \in O_{2} \backslash O$-in which case the proof is finished-or $u_{1} \in O$, so we may assume that $u_{1}=v_{1}$ without loss of generality. But then, since $O \subseteq O_{1}$, we know that $u_{2}-v_{1} \in D$ for some $u_{2}$ in $O_{2}$. By coherence of $D$ [Axiom $\mathrm{D}_{1}$ ], we know that $u_{2} \neq v_{1}$, and therefore also that $u_{2}$ in $O_{2} \backslash\left\{v_{1}\right\}$. Then either $u_{2} \in O_{2} \backslash O$ in which case the proof is finished-or $u_{2} \in O \backslash\left\{v_{1}\right\}$, so we may assume that $u_{2}=v_{2}$ without loss of generality. This tells us that $v_{2}-v_{1} \in D$. But then, again, since $O \subseteq O_{1}$, we know that $u_{3}-v_{2} \in D$ for some $u_{3}$ in $O_{2}$. The coherence of $D$ [Axiom $\left.\mathrm{D}_{4}\right]$ then implies that also $u_{3}-v_{1}=u_{3}-v_{2}+v_{2}-v_{1} \in D$, and hence that [use Axiom $\mathrm{D}_{1}$ twice] $u_{3} \notin\left\{v_{1}, v_{2}\right\}$, so we know that $u_{3}$ in $O_{2} \backslash\left\{v_{1}, v_{2}\right\}$. When we proceed in this way, we are guaranteed to find, after $k \leq n+1$ steps, some $u_{k}$ in $\mathrm{O}_{2} \backslash \mathrm{O}$ such that $u_{k}-u \in D$.

$\mathrm{C}_{4}$ Consider any $O_{1}, O_{2}$ in $\mathcal{Q}$ such that $O_{1} \subseteq C_{D}\left(O_{2}\right)$, meaning that $\left(\forall u \in O_{1}\right)(\forall v \in$ $\left.\mathrm{O}_{2}\right) v-u \notin D$.

$\mathrm{C}_{4}$ a It follows that $\left(\forall u \in O_{1}\right)\left(\forall v \in O_{2}\right) \lambda v-\lambda u \notin D$ for any $\lambda$ in $\mathbb{R}_{>0}$, whence indeed $\lambda O_{1} \subseteq C_{D}\left(\lambda O_{2}\right)$.

$\mathrm{C}_{4} \mathrm{~b}$ Also $\left(\forall u \in O_{1}\right)\left(\forall v \in O_{2}\right)(v+w)-(u+w) \notin D$ for any $w$ in $\mathcal{V}$, whence indeed $O_{1}+\{w\} \subseteq C_{D}\left(O_{2}+\{w\}\right)$

(c) Finally, we show that $C_{D} \sqsubseteq C$ for all $C \in \overline{\mathcal{C}}_{D}$ : Consider any $C$ in $\overline{\mathcal{C}}_{D}$ and $O$ in $\mathcal{Q}$, then we have to prove that $C(O) \subseteq C_{D}(O)$. Consider any $u$ in $O$ and assume that $u \notin C_{D}(O)$. Then $v-u \in D$ for some $v$ in $O$, implying that $u \notin C(\{u, v\})$ because $C$ is compatible with $D$. The coherence of $C$ [version (3) of Axiom $\mathrm{C}_{3}$ a with $\tilde{O}_{1}:=\{u\}, \tilde{O}_{2}:=\{u, v\}$ and $\tilde{O}:=O$ ] then implies that also $u \notin C(O)$.

Proof of Proposition 14. Because $D_{\mathrm{v}} \in \overline{\mathcal{D}}$, we have that $C_{D_{\mathrm{v}}}$ is a coherent choice function by Proposition 13 , and it then follows that $C_{\mathrm{v}} \sqsubseteq C_{D_{\mathrm{v}}}$. We show that $C_{D_{\mathrm{v}}} \sqsubseteq C_{\mathrm{v}}$, or in other words, that

$$
(\forall O \in \mathcal{Q})(\forall u \in O) u \notin C_{D_{\mathrm{v}}}(O) \Rightarrow u \notin C_{\mathrm{v}}(O)
$$

Fix any $O$ in $\mathcal{Q}$ and any $u$ in $O$ such that $u \notin C_{D_{\mathrm{v}}}(O)$, meaning that $u<v$ for some $v$ in $O$ by the definition of $C_{D_{\mathrm{v}}}$. Then, due to Axiom $\mathrm{C}_{2}$, we find that $u \notin C(\{u, v\})$, and using version (3) of Axiom $\mathrm{C}_{3} \mathrm{a}$ [with $\tilde{O}_{1}:=\{u\}, \tilde{O}_{2}:=\{u, v\}$ and $\tilde{O}:=O$ ] we infer that $u \notin C_{\mathrm{v}}(O)$.

Proof of Proposition 15. Recall in advance that inf $\mathcal{C}^{\prime}$ is a coherent choice function by Proposition 8 , and that $\inf \left\{D_{C}: C \in \mathcal{C}^{\prime}\right\}$ is a coherent set of desirable options by Proposition 9

For the first statement, consider any $u$ in $\mathcal{V}$, and observe that

$$
\begin{aligned}
u \in D_{\text {inf } \mathcal{C}^{\prime}} & \Leftrightarrow 0 \notin \inf \mathcal{C}^{\prime}(\{0, u\}) \Leftrightarrow 0 \notin \bigcup_{C \in \mathcal{C}^{\prime}} C(\{0, u\}) \\
& \Leftrightarrow\left(\forall C \in \mathcal{C}^{\prime}\right) 0 \notin C(\{0, u\}) \\
& \Leftrightarrow\left(\forall C \in \mathcal{C}^{\prime}\right) u \in D_{C} \\
& \Leftrightarrow u \in \bigcap\left\{D_{C}: C \in \mathcal{C}^{\prime}\right\}=\inf \left\{D_{C}: C \in \mathcal{C}^{\prime}\right\},
\end{aligned}
$$

where the first and fourth equivalences follow from Proposition 12 .

For the second statement, fix any $O$ in $\mathcal{Q}$ and $u$ in $\mathcal{V}$ such that $u \in \inf \mathcal{C}^{\prime}(O)$. Then $u \in C(O)$ for some $C$ in $\mathcal{C}^{\prime}$, from which we infer that $\left(\exists C \in \mathcal{C}^{\prime}\right)(\forall v \in O) u \in C(\{u, v\})$, by an immediate application of version (3) [use the contraposition with $\tilde{O}_{1}:=\{u\}, \tilde{O}_{2}:=\{u, v\}$ and $\tilde{O}:=O]$ of Axiom $\mathrm{C}_{3}$ a By exchanging the quantifiers, we infer as an implication that 
$(\forall v \in O)\left(\exists C \in \mathcal{C}^{\prime}\right) u \in C(\{u, v\})$. Now recall that

$$
\begin{aligned}
u \in C(\{u, v\}) & \Leftrightarrow 0 \in C(\{0, v-u\}) & & \text { by Axiom } \mathrm{C}_{4} \mathrm{~b} \\
& \Leftrightarrow v-u \notin D_{C} & & \text { by Proposition } 12 .
\end{aligned}
$$

This implies that $(\forall v \in O) v-u \notin \bigcap\left\{D_{C}: C \in \mathcal{C}^{\prime}\right\}$, which is equivalent to $u \in C_{\inf \left\{D_{C}: C \in \mathcal{C}^{\prime}\right\}}(O)$, by Proposition 13 . The rest of the proof is now immediate.

Proof of Proposition 16. Recall in advance that $\inf \left\{C_{D}: D \in \mathcal{D}^{\prime}\right\}$ is a coherent choice function by Propositions 8 and 13 , and that $\inf ^{\prime}$ is a coherent set of desirable options by Proposition 9

For the first statement, consider any $u$ in $\mathcal{V}$ and observe that:

$$
\begin{array}{rlrl}
u \in D_{\inf \left\{C_{D}: D \in \mathcal{D}^{\prime}\right\}} & \Leftrightarrow 0 \notin\left(\inf \left\{C_{D}: D \in \mathcal{D}^{\prime}\right\}\right)(\{0, u\})=\bigcup_{D \in \mathcal{D}^{\prime}} C_{D}(\{0, u\}) & & \text { by Pr. 12 } \\
& \Leftrightarrow\left(\forall D \in \mathcal{D}^{\prime}\right) 0 \notin C_{D}(\{0, u\}) & \\
& \Leftrightarrow\left(\forall D \in \mathcal{D}^{\prime}\right)(\exists v \in\{0, u\}) v \in D & & \text { by Pr.13 } \\
& \Leftrightarrow\left(\forall D \in \mathcal{D}^{\prime}\right) u \in D & & \text { by Ax. } \mathbb{D}_{1} \\
& \Leftrightarrow u \in \inf \mathcal{D}^{\prime} . &
\end{array}
$$

For the second statement, let $\mathcal{C}^{\prime}:=\left\{C_{D}: D \in \mathcal{D}^{\prime}\right\}$, then we infer from the first statement that $\left\{D_{C}: C \in \mathcal{C}^{\prime}\right\}=\mathcal{D}^{\prime}$. Now, use the second statement in Proposition 15 .

Proof of Corollary 17. It is an immediate consequence of Propositions 16 and 15, by letting $\mathcal{D}^{\prime}:=\{D\}$ and $\mathcal{C}^{\prime}:=\{C\}$.

Proof of Proposition 18 Immediate consequence of Propositions 8 and 13

Proof of Proposition 19 Consider any $u$ in $\mathcal{V}$, then

$$
\begin{aligned}
u \in D_{C_{D^{\prime}}^{\mathrm{M}}} & \Leftrightarrow 0 \notin C_{D^{\prime}}^{\mathrm{M}}(\{0, u\}) & & \text { by Proposition } 12 \\
& \Leftrightarrow\left(\forall \hat{D} \in \hat{\mathcal{D}}_{D^{\prime}}\right) 0 \notin C_{\hat{D}}(\{0, u\}) & & \text { by Definition 11 } \\
& \Leftrightarrow\left(\forall \hat{D} \in \hat{\mathcal{D}}_{D^{\prime}}\right) u \in \hat{D} & & \text { by Proposition } 12 \\
& \Leftrightarrow u \in \bigcap \hat{\mathcal{D}}_{D^{\prime}} \Leftrightarrow u \in D^{\prime} & & \text { by Proposition } 11
\end{aligned}
$$

proving the first statement. It then follows from Proposition 12 that $D^{\prime}$ is compatible with $C_{D^{\prime}}^{\mathrm{M}}$, and therefore from Proposition 13 that $C_{D^{\prime}}=\inf \overline{\mathcal{C}}_{D^{\prime}} \subseteq C_{D^{\prime}}^{\mathrm{M}}$.

Proof of Proposition 20. By Proposition 13, it suffices to prove (a) that $D_{p}$ is coherent; and (b) that $C_{p}=C_{D_{p}}$.

For (a), that Axiom $\mathrm{D}_{1}$ holds, follows from $0 \notin \mathcal{V}_{>0}$ and $\mathrm{E}_{p}(0)=0$. Axiom $\mathrm{D}_{2}$ holds by definition. For Axiom $\mathrm{D}_{3}$, consider any $u$ in $D_{p}$ and real $\lambda$ in $\mathbb{R}_{>0}$, then $0<u$ and therefore $0<\lambda u$, or $\mathrm{E}_{p}(u)>0$ and therefore $\mathrm{E}_{p}(\lambda u)=\lambda \mathrm{E}_{p}(u)>0$, whence indeed $\lambda u \in D_{p}$. For Axiom $\mathrm{D}_{4}$, consider any $u, v$ in $D_{p}$, then there are three possibilities. The first is that both $u$ and $v$ belong to $\mathcal{V}_{>0}$, and therefore also $u+v \in \mathcal{V}_{>0}$. The second is that both $\mathrm{E}_{p}(u)>0$ and $\mathrm{E}_{p}(v)>0$, and therefore also $\mathrm{E}_{p}(u+v)=\mathrm{E}_{p}(u)+\mathrm{E}_{p}(v)>0$. And the third is that, without loss of generality, $\mathrm{E}_{p}(u)>0$ and $v \in \mathcal{V}_{>0}$, whence $\mathrm{E}_{p}(v) \geq 0$ and therefore $\mathrm{E}_{p}(u+v)=\mathrm{E}_{p}(u)+\mathrm{E}_{p}(v)>0$. In all cases therefore indeed $u+v \in D_{p}$.

For (b), consider any $O$ in $\mathcal{Q}$, use Equation $(8)$ to find that $C_{D_{p}}(O)=\{u \in O:(\forall v \in$ $\left.O) v-u \notin D_{p}\right\}$ and Equation (10) to find that $v-u \notin D_{p} \Leftrightarrow\left(v-u \notin \mathcal{V}_{>0}\right.$ and $\left.\mathrm{E}_{p}(v-u) \leq 0\right)$. It then follows from Equation (11) that indeed $C_{p}(O)=C_{D_{p}}(O)$. 
Proof of Proposition 21. We infer from Definition 12 and Proposition 20 that

$$
C_{K}^{\mathrm{E}}(O)=\bigcup_{p \in K} C_{D_{p}}(O)=\bigcup_{p \in K}\left\{u \in O:(\forall v \in O) v-u \notin D_{p}\right\},
$$

where the last equality follows from Proposition 13 Now

$$
\begin{aligned}
(\forall v \in O) v-u \notin D_{p} & \Leftrightarrow(\forall v \in O)\left(u \nless v \text { and } \mathrm{E}_{p}(v-u) \leq 0\right) \\
& \left.\Leftrightarrow u \in C_{\mathrm{v}}(O) \text { and }(\forall v \in O) \mathrm{E}_{p}(v-u) \leq 0\right),
\end{aligned}
$$

where the first equivalence follows from Equation $(10)$, and the second from Proposition 14 Hence indeed

$$
\begin{aligned}
C_{K}^{\mathrm{E}}(O) & =\bigcup_{p \in K}\left\{u \in O:(\forall v \in O) \mathrm{E}_{p}(v-u) \leq 0\right\} \cap C_{\mathrm{v}}(O) \\
& =\left\{u \in O:(\exists p \in K)(\forall v \in O) \mathrm{E}_{p}(v) \leq \mathrm{E}_{p}(u)\right\} \cap C_{\mathrm{v}}(O) .
\end{aligned}
$$

Proof of Proposition 22. We consider any $p$ in $K$ and prove that $C_{D_{p}} \sqsubseteq C_{\hat{\mathcal{D}}_{\{p\}}}^{\mathrm{M}}$. The proof then follows by taking the infimum $\inf _{p \in K}$ over $p \in K$ on both sides of this inequality. Consider any $O$ in $\mathcal{Q}$ and assume that $u \in C_{\hat{\mathcal{D}}_{\{p\}}^{\mathrm{M}}}(O)$, so there is some $\hat{D}$ in $\hat{\mathcal{D}}_{\{p\}}$ such that $u \in C_{\hat{D}}(O)$, or equivalently, $(\forall v \in O) v-u \notin \hat{D}$. Hence $(\forall v \in O) v-u \notin D_{p}$, because $D_{p} \subseteq \hat{D}$, and therefore indeed $u \in C_{D_{p}}(O)$.

Proof of Proposition 23. To see that $\leq$ is reflexive, use $w=0$ in Equation (13). For antisymmetry, consider any $[u],[v]$ in $\mathcal{V} / I$ such that $[u] \leq[v]$ and $[v] \leq[u]$, and therefore $u \leq v+w$ and $v \leq u+w^{\prime}$ for some $w, w^{\prime} \in I$. This implies, by repeatedly applying Equation (1) and the transitivity of the vector ordering on $\mathcal{V}$ that $w+w^{\prime} \geq 0$. Now, by Axiom $\mathrm{I}_{4}, w+w^{\prime} \in I$, and therefore, by Axiom $\Pi_{2} w+w^{\prime}=0$, implying that $u \leq v+w$ and $v \leq u-w$, and therefore also $v+w \leq u$, for some $w \in I$. Hence indeed $u=v+w$ and therefore $[u]=[v]$, by the antisymmetry of the vector ordering on $\mathcal{V}$. For transitivity, consider any $[u],[v],[w]$ in $\mathcal{V} / I$ such that $[u] \leq[v]$ and $[v] \leq[w]$, and therefore $u \leq v+v^{\prime}$ and $v \leq w+w^{\prime}$ for some $v^{\prime}, w^{\prime} \in I$. Then $v+v^{\prime} \leq w+w^{\prime}+v^{\prime}$ by Equation (1), whence also $u \leq w+w^{\prime}+v^{\prime}$ by transitivity of the vector ordering on $\mathcal{V}$. By coherence $\left[\right.$ Axiom $\left[I_{4}\right]$ also $v^{\prime}+w^{\prime} \in I$, whence indeed $[u] \leq[w]$ by Equation (13).

Next, we prove that $\leq$ satisfies Eqs. (1) and (2). Consider any $\left[u_{1}\right],\left[u_{2}\right]$ in $\mathcal{V} / I$ such that $\left[u_{1}\right] \leq\left[u_{2}\right]$, implying that $u_{1} \leq u_{2}+u_{2}^{\prime}$ for some $u_{2}^{\prime} \in I$. For (1), consider any $v$ in $\mathcal{V}$, then also $u_{1}+v \leq u_{2}+v+u_{2}^{\prime}$, implying that indeed $\left[u_{1}\right]+[v] \leq\left[u_{2}\right]+[v]$. The converse implication follows analogously, by adding $-v$ rather than $v$. For $[2]$, consider any $\lambda$ in $\mathbb{R}_{>0}$, then also $\lambda u_{1} \leq \lambda u_{2}+\lambda u_{2}^{\prime}$, implying that indeed $\lambda\left[u_{1}\right] \leq \lambda\left[u_{2}\right]$ because $\lambda u_{2}^{\prime} \in I$ by Axiom $I_{3}$. The converse implication follows analogously, by multiplying with $\frac{1}{\lambda}$ rather than $\lambda$.

We turn to the second statement. For necessity, consider $u, v$ in $\mathcal{V}$ such that $[u]<[v]$, so $[u] \neq[v]$ and $u \leq v+w$ for some $w$ in $I .[u] \neq[v]$ implies that $u \neq v+w$ for all $w$ in $I$. Taken together with $u \leq v+w$ for some $w$, this implies that indeed $u<v+w$ for some $w$. For sufficiency, consider $u, v$ in $\mathcal{V}$ such that $u<v+w$ for some $w$ in $I$. Assume ex absurdo that $[u] \nless[v]$, meaning that $[u]=[v]$ or $u \neq v+w$ for all $w$ in $I$. The latter possibility is incompatible with the assumption that $u<v+w$ for some $w$ in $I$, leaving only the first possibility, which is equivalent to $u=v+w^{\prime}$ for some $w^{\prime}$ in $I$. Then $v+w^{\prime}<v+w$, implying that $0<w-w^{\prime}$ by Equation (1). This contradicts the coherence of $I$ [Axiom [ $I_{2}$ ] because $w-w^{\prime}$ is an element of $I$ by Axiom $I_{4}$.

Proof of Proposition 24. For necessity, observe that

$$
D+I=\{u+v: u \in D \text { and } v \in I\}=\bigcup\{\{u\}+I: u \in D\}=\bigcup\{[u]: u \in D\}=\bigcup D / I,
$$


and that compatibility with $I$ guarantees that $D+I=D$. For sufficiency, assume that there is some set of desirable options $D^{\prime} \subseteq \mathcal{V} / I$ such that $D=\left\{u:[u] \in D^{\prime}\right\}$. Consider any $u$ in $D$ and any $v$ in $I=[0]$, then $u+v \in[u]+[0]=[u+0]=[u]$, and therefore indeed $u+v \in D$. Then also $D / I=\{[u]: u \in D\}=\left\{[u]:[u] \in D^{\prime}\right\}=D^{\prime}$.

Proof of Proposition 25. For the direct implication, assume that $D \subseteq \mathcal{V}$ is coherent. We show that $D / I=\{[u]: u \in D\} \subseteq \mathcal{V} / I$ satisfies the Axioms $\mathrm{D}_{1} \mathrm{D}_{4}$. For Axiom $\mathrm{D}_{1}$, assume ex absurdo that $[0] \in D / I$, implying that $u \in D$ for some $u$ in $[0]=I$, but then $u \in D \cap I$ and hence $D \cap I$ is non-empty, a contradiction. For Axiom $\mathrm{D}_{2}$, consider any $[u]$ in $\mathcal{V} / I$ such that $[0]<[u]$, meaning that $0<u+w$ for some $w$ in $I$. Then $u+w \in D$ for some $w$ in $I$, by coherence of $D$ [Axiom $\left.\mathrm{D}_{2}\right]$, implying that $[u]=[u]+I=[u]+[w]=[u+w] \in D / I$. For Axiom $\mathrm{D}_{3}$, assume that $[u] \in D / I$, and consider any $\lambda$ in $\mathbb{R}_{>0}$. Then $u \in D$, implying that $\lambda u \in D$ by coherence of $D\left[\right.$ Axiom $\left.\mathrm{D}_{3}\right]$, whence $\lambda[u]=[\lambda u] \in D / I$. For Axiom $\mathrm{D}_{4}$ assume that $[u],[v] \in D / I$, implying that $u, v \in D$. Then $u+v \in D$ by coherence of $D$ [Axiom $\mathrm{D}_{4}$ ], whence $[u]+[v]=[u+v] \in D / I$.

For the converse implication, assume that $D / I \subseteq \mathcal{V} / I$ is coherent. We show that $D=\{u$ : $[u] \in D / I\} \subseteq \mathcal{V}$ satisfies the Axioms $\mathrm{D}_{1}, \mathrm{D}_{4}$. For Axiom $\mathrm{D}_{1}$, infer that $0 \notin D$ since $[0] \notin D / I$ by coherence of $D / I$ [Axiom $\left[\mathrm{D}_{1}\right]$. For Axiom $\mathrm{D}_{2}$, consider any $u$ in $\mathcal{V}$ such that $0<u$. Then $[0]<[u]$ by taking for example $w=0$ in Proposition 23 , implying that $[u] \in D / I$ by coherence of $D / I$ [Axiom $\left.\mathrm{D}_{2}\right]$, whence $u \in D$. For Axiom $\mathrm{D}_{3}$, assume that $u \in D$, and consider any $\lambda$ in $\mathbb{R}_{>0}$. Then $[u] \in D / I$, implying that $\lambda[u]=[\lambda u] \in D / I$ by coherence of $D / I\left[\right.$ Axiom $\mathrm{D}_{3}$, whence $\lambda u \in D$. For Axiom $\mathrm{D}_{4}$, assume that $u, v \in D$, implying that $[u],[v] \in D / I$. Then $[u]+[v] \in D / I$ by coherence of $D / I\left[\right.$ Axiom $\left.\mathrm{D}_{4}\right]$, implying that $u+v \in D$.

Proof of Proposition 26. For the first statement, assume that $C$ is compatible with $I$, that is, there is some $C^{\prime}$ on $\mathcal{Q}(\mathcal{V} / I)$ such that $C(O):=\left\{u \in O:[u] \in C^{\prime}(O / I)\right\}$ for all $O$ in $\mathcal{Q}(\mathcal{V})$. Consider any $O_{1}$ and $O_{2}$ in $\mathcal{Q}(\mathcal{V})$. Infer that

$$
\begin{aligned}
O_{1} \triangleleft_{C} O_{2} \Leftrightarrow O_{1} \cap C\left(O_{1} \cup O_{2}\right)=\varnothing & \Leftrightarrow O_{1} \cap\left\{u \in O_{1} \cup O_{2}:[u] \in C^{\prime}\left(\left(O_{1} \cup O_{2}\right) / I\right)\right\}=\varnothing \\
& \Leftrightarrow O_{1} / I \cap C^{\prime}\left(\left(O_{1} \cup O_{2}\right) / I\right)=\varnothing \\
& \Leftrightarrow O_{1} / I \triangleleft_{C^{\prime}} O_{2} / I .
\end{aligned}
$$

This shows that the representing choice relation is derived from the representing choice function.

For the second statement, assume that $\triangleleft$ is compatible with $I$, that is, there is some $\triangleleft^{\prime}$ on $\mathcal{Q}(\mathcal{V} / I)$ such that $O_{1} \triangleleft O_{2} \Leftrightarrow O_{1} / I \triangleleft^{\prime} O_{2} / I$ for all $O_{1}$ and $O_{2}$ in $\mathcal{Q}(\mathcal{V})$. Consider any $O$ in $\mathcal{Q}(\mathcal{V})$. Then $C_{\triangleleft}(O)=O \backslash \bigcup\left\{O^{\prime} \subseteq O: O^{\prime} \triangleleft O\right\}=O \backslash \bigcup\left\{O^{\prime} \subseteq O: O^{\prime} / I \triangleleft^{\prime} O / I\right\}$. Infer that

$$
\begin{aligned}
u \in C_{\triangleleft}(O) & \Leftrightarrow u \in O \text { and } u \notin \bigcup\left\{O^{\prime} \subseteq O: O^{\prime} / I \triangleleft^{\prime} O / I\right\} \\
& \Leftrightarrow u \in O \text { and }[u] \notin \bigcup\left\{\tilde{O} \subseteq O / I: \tilde{O} \triangleleft^{\prime} O / I\right\} \\
& \Leftrightarrow u \in O \text { and }[u] \in O / I \backslash \bigcup\left\{\tilde{O} \subseteq O / I: \tilde{O} \triangleleft^{\prime} O / I\right\} \\
& \Leftrightarrow u \in O \text { and }[u] \in C_{\triangleleft^{\prime}}(O / I),
\end{aligned}
$$

whence $C_{\triangleleft}$ is indeed compatible with $I$, with representing choice function $C_{\triangleleft^{\prime}}$.

Proof of Proposition 27. Let $C^{\prime}$ be any representing choice function, and consider any $O$ in $\mathcal{Q}(\mathcal{V})$, then

$$
C(O) / I=\{[u]: u \in C(O)\}=\left\{[u]: u \in O,[u] \in C^{\prime}(O / I)\right\}=O / I \cap C^{\prime}(O / I)=C^{\prime}(O / I) .
$$

Lemma 36. Given any two option sets $O_{1}, O_{2}$ in $\mathcal{Q}(\mathcal{V})$, then $O_{2} / I \backslash O_{1} / I \subseteq\left(O_{2} \backslash O_{1}\right) / I$. 
Proof. Consider any $\tilde{u}$ in $O_{2} / I \backslash O_{1} / I$, then $\tilde{u}=\left[v_{2}\right]$ for some $v_{2}$ in $O_{2}$ and $\tilde{u} \neq\left[v_{1}\right]$ for all $v_{1}$ in $O_{1}$, implying that indeed $\tilde{u}=\left[v_{2}\right]$ for some $v_{2}$ in $O_{2} \backslash O_{1}$.

Lemma 37. For any choice function $C$ on $\mathcal{Q}(\mathcal{V})$ that is compatible with a coherent set of indifferent options $I$, and any option set $O$ in $\mathcal{Q}(\mathcal{V})$, we have that $(O \backslash C(O)) / I=$ $O / I \backslash C / I(O / I)$.

Proof. Consider any $\tilde{u}$ in $\mathcal{V} / I$, then

$$
\begin{aligned}
\tilde{u} \in(O \backslash C(O)) / I & \Leftrightarrow(\exists v \in O \backslash C(O)) \tilde{u}=[v] \\
& \Leftrightarrow(\exists v \in O)(\tilde{u}=[v] \text { and } v \notin C(O)) \\
& \Leftrightarrow(\exists v \in O)(\tilde{u}=[v] \text { and }[v] \notin C / I(O / I)) \quad \text { by Proposition 27 } \\
& \Leftrightarrow(\exists v \in O)(\tilde{u}=[v] \text { and } \tilde{u} \notin C / I(O / I)) \\
& \Leftrightarrow \tilde{u} \notin C / I(O / I) \text { and }(\exists v \in O) \tilde{u}=[v] \\
& \Leftrightarrow \tilde{u} \in O / I \backslash C / I(O / I),
\end{aligned}
$$

whence indeed $(O \backslash C(O)) / I=O / I \backslash C / I(O / I)$.

Lemma 38. For any choice function $C$ on $\mathcal{Q}(\mathcal{V})$ that is compatible with a coherent set of indifferent options $I$, and any option sets $O_{1}, O_{2}$ in $\mathcal{Q}(\mathcal{V})$ such that $O_{1} \subseteq O_{2}$, we have that $O_{1} \cap C\left(O_{2}\right)=\varnothing \Leftrightarrow O_{1} / I \cap C / I\left(O_{2} / I\right)=\varnothing$.

Proof. First, assume that $O_{1} \cap C\left(O_{2}\right) \neq \varnothing$, and consider any $v$ in $O_{1} \cap C\left(O_{2}\right)$. Then $[v] \in$ $O_{1} / I$ and $[v] \in C\left(O_{2}\right) / I=C / I\left(O_{2} / I\right)$, by Proposition 27. Conversely, assume that $O_{1} / I \cap$ $C / I\left(O_{2} / I\right) \neq \varnothing$, and consider any $\tilde{u}$ in $O_{1} / I \cap C / I\left(O_{2} / I\right)$. Then there is some $v$ in $O_{1}$ such that $\tilde{u}=[v]$, and we infer from Proposition 27 that $\tilde{u} \in C\left(O_{2}\right) / I$, so also $v \in C\left(O_{2}\right)$.

Lemma 39. For all $\tilde{O}$ in $\mathcal{Q}(\mathcal{V} / I)$, there is some $O$ in $\mathcal{Q}(\mathcal{V})$ such that $O / I=\tilde{O}$.

Proof. For every element $\tilde{u}$ in the finite set $\tilde{O}$, we take some vector $u$ in $\mathcal{V}$ such that $[u]=\tilde{u}$, and collect those vectors in an option set $O$. Then $O \in \mathcal{Q}(\mathcal{V})$ because it is a finite subset of $\mathcal{V}$, and $O / I=\{[u]: u \in O\}=\tilde{O}$.

Proof of Proposition 28. For the direct implication, assume that $C$ is coherent. We show that $C / I$ satisfies Axioms $\mathrm{C}_{1}-\mathrm{C}_{4} \mathrm{~b}$.

For Axiom $\mathrm{C}_{1}$, assume ex absurdo that $C / I(\tilde{O})=\varnothing$ for some $\tilde{O}$ in $\mathcal{Q}(\mathcal{V} / I)$. Consider any option set $O$ in $\mathcal{Q}(\mathcal{V})$ such that $O / I=\tilde{O}$, then $C(O)=\{u \in O:[u] \in C / I(\tilde{O})\}=\{u \in O$ : $[u] \in \varnothing\}=\varnothing$, contradicting the coherence of $C\left[\right.$ Axiom $\left.\mathrm{C}_{1}\right]$.

For Axiom $\mathrm{C}_{2}$, consider $[u],[v]$ in $\mathcal{V} / I$ such that $[u]<[v]$, meaning that $u<v+w$ for some $w$ in $I$, by Proposition 23. Then $\{v+w\}=C(\{u, v+w\})$ by coherence of $C$ [Axiom $\mathrm{C}_{2}$, so $\{[v]\}=\{[v+w]\}=C / I(\{[u],[v+w]\})=C / I(\{[u],[v]\})$, where the first and last equalities hold because $w$ belongs to $I$.

For version (3) of Axiom $\mathrm{C}_{3}$ a consider any option sets $\tilde{O}_{1}, \tilde{O}_{2}, \tilde{O}$ in $\mathcal{Q}(\mathcal{V} / I)$ such that $\tilde{O}_{1} \cap C / I\left(\tilde{O}_{2}\right)=\varnothing$ and $\tilde{O}_{1} \subseteq \tilde{O}_{2} \subseteq \tilde{O}$. Consider any option set $O$ in $\mathcal{Q}(\mathcal{V})$ such that $O / I=\tilde{O}$, and define the option sets $O_{2}:=\left\{u \in O:[u] \in \tilde{O}_{2}\right\} \in \mathcal{Q}(\mathcal{V})$ and $O_{1}:=\left\{u \in O_{2}:[u] \in \tilde{O}_{1}\right\} \in$ $\mathcal{Q}(\mathcal{V})$. Then $O_{1} \subseteq O_{2} \subseteq O, O_{2} / I=\left\{[v]: v \in O_{2}\right\}=\left\{[v]: v \in O\right.$ and $\left.[v] \in \tilde{O}_{2}\right\}=O / I \cap\{[v]:$ $\left.[v] \in \tilde{O}_{2}\right\}=\tilde{O} \cap \tilde{O}_{2}=\tilde{O}_{2}$ and, similarly, $O_{1} / I=\left\{[v]: v \in O_{2}\right.$ and $\left.[v] \in \tilde{O}_{1}\right\}=O_{2} / I \cap \tilde{O}_{1}=$ $\tilde{O}_{2} \cap \tilde{O}_{1}=\tilde{O}_{1}$, so we infer from Lemma 38 that $O_{1} \cap C\left(O_{2}\right)=\varnothing$, and therefore also that $O_{1} \cap C(O)=\varnothing$ by coherence of $C$ [version (3) of Axiom $\mathrm{C}_{3}$ a]. Because $\tilde{O}=O / I$, we conclude from Lemma 38 that indeed $\tilde{O}_{1} \cap C / I(\tilde{O})=\varnothing$.

For version 4 of Axiom $\mathrm{C}_{3} \mathrm{~b}$, consider any option sets $\tilde{O}_{1}, \tilde{O}_{2}, \tilde{O}$ in $\mathcal{Q}(\mathcal{V} / I)$ such that $\tilde{O}_{1} \cap C / I\left(\tilde{O}_{2}\right)=\varnothing$ and $\tilde{O} \subseteq \tilde{O}_{1}$. Let $\tilde{O}_{1}^{\prime}:=\tilde{O}_{1} \cap \tilde{O}_{2}$ and $\tilde{O}_{1}^{\prime \prime}:=\tilde{O}_{1} \backslash \tilde{O}_{2}$, then $\tilde{O}=\tilde{O}^{\prime} \cup \tilde{O}^{\prime \prime}$, 
and $\tilde{O}^{\prime} \subseteq \tilde{O}_{1}^{\prime} \subseteq \tilde{O}_{2}$. So the assumption $\tilde{O}_{1} \cap C / I\left(\tilde{O}_{2}\right)=\varnothing$ implies that $\tilde{O}_{1}^{\prime} \cap C / I\left(\tilde{O}_{2}\right)=\varnothing$. Introduce the option sets $O^{\prime}, O_{1}^{\prime}, O_{2}$ in $\mathcal{Q}(\mathcal{V})$ as follows: $O_{2}$ is any option set such that $O_{2} / I=\tilde{O}_{2}, O_{1}^{\prime}:=\left\{u \in O_{2}:[u] \in \tilde{O}_{1}^{\prime}\right\}$ and $O^{\prime}:=\left\{u \in O_{1}^{\prime}:[u] \in \tilde{O}^{\prime}\right\}=\left\{u \in O_{2}:[u] \in \tilde{O}^{\prime}\right\}$, then $O^{\prime} \subseteq O_{1}^{\prime} \subseteq O_{2}$. Also, $O_{1}^{\prime} / I=\tilde{O}_{1}^{\prime}, O^{\prime} / I=\tilde{O}^{\prime}$, and $\tilde{O}_{1}^{\prime} \cap C / I\left(\tilde{O}_{2}\right)=\varnothing$ allows us to infer that $O_{1}^{\prime} \cap C\left(O_{2}\right)=\varnothing$, using Lemma 38. The coherence of $C$ [version (4) of Axiom $\mathrm{C}_{3}$ b] then implies that $O_{1}^{\prime} \cap C\left(O_{2} \backslash O^{\prime}\right)=\varnothing$, and therefore also $\left(O_{1}^{\prime} \backslash O^{\prime}\right) \cap C\left(O_{2} \backslash O^{\prime}\right)=\varnothing$. This implies that $O_{1}^{\prime} \backslash O^{\prime} \subseteq\left(O_{2} \backslash O^{\prime}\right) \backslash C\left(O_{2} \backslash O^{\prime}\right)$, because $O_{1}^{\prime} \backslash O^{\prime} \subseteq O_{2} \backslash O^{\prime}$. By Equation (14) and Lemma 37 this implies that $\left(O_{1}^{\prime} \backslash O^{\prime}\right) / I \subseteq\left(O_{2} \backslash O^{\prime}\right) / I \backslash C / I\left(\left(O_{2} \backslash O^{\prime}\right) / I\right)$. We now prove that

$$
\left(O_{1}^{\prime} \backslash O^{\prime}\right) / I=O_{1}^{\prime} / I \backslash O^{\prime} / I
$$

and that

$$
\left(O_{2} \backslash O^{\prime}\right) / I=O_{2} / I \backslash O^{\prime} / I
$$

The reasoning is similar for both equalities, and therefore we restrict ourselves here to proving the first one; the second one can be proved by replacing $O_{2}$ by $O_{1}^{\prime}$. That $\left(O_{1}^{\prime} \backslash O^{\prime}\right) / I \supseteq O_{1}^{\prime} / I \backslash O^{\prime} / I$ follows from Lemma 36 . To show that $\left(O_{1}^{\prime} \backslash O^{\prime}\right) / I \subseteq O_{1}^{\prime} / I \backslash O^{\prime} / I$, consider any $\tilde{u}$ in $\left(O_{1}^{\prime} \backslash O^{\prime}\right) / I$, meaning that $\tilde{u}=\left[v_{1}\right]$ for some $v$ in $O_{1}^{\prime} \backslash O^{\prime}$. That implies already that $\tilde{u} \in O_{1}^{\prime} / I$. Assume ex absurdo that $\tilde{u} \in O^{\prime} / I=\tilde{O}^{\prime}$, and consider any $v \in O_{1}^{\prime}$ such that $[v]=\tilde{u}$, then also $v \in O_{2}$ and $[v] \in \tilde{O}^{\prime}$, and therefore $v \in O^{\prime}$. But this implies that $\tilde{u} \notin\left(O_{1}^{\prime} \backslash O^{\prime}\right) / I$, a contradiction. So we conclude that $\left(O_{1}^{\prime} \backslash O^{\prime}\right) / I=O_{1}^{\prime} / I \backslash O^{\prime} / I$ and that $\left(O_{2} \backslash O^{\prime}\right) / I=O_{2} / I \backslash O^{\prime} / I$, implying that

$$
\tilde{O}_{1}^{\prime} \backslash \tilde{O}^{\prime}=O_{1}^{\prime} / I \backslash O^{\prime} / I \subseteq\left(O_{2} / I \backslash O^{\prime} / I\right) \backslash C / I\left(O_{2} / I \backslash O^{\prime} / I\right)=\left(\tilde{O}_{2} \backslash \tilde{O}^{\prime}\right) \backslash C / I\left(\tilde{O}_{2} \backslash \tilde{O}^{\prime}\right),
$$

or equivalently, $\left(\tilde{O}_{1}^{\prime} \backslash \tilde{O}^{\prime}\right) \cap C / I\left(\tilde{O}_{2} \backslash \tilde{O}^{\prime}\right)=\varnothing$. Since $\tilde{O}^{\prime} \cap C / I\left(\tilde{O}_{2} \backslash \tilde{O}^{\prime}\right)=\varnothing$, this also implies that $\tilde{O}_{1}^{\prime} \cap C / I\left(\tilde{O}_{2} \backslash \tilde{O}^{\prime}\right)=\varnothing$. Since $\tilde{O}_{2} \backslash \tilde{O}^{\prime}=\tilde{O}_{2} \backslash \tilde{O}$ and $\tilde{O}_{1} \cap \tilde{O}_{2}=\tilde{O}_{1}^{\prime}$, this indeed leads to $\varnothing=\tilde{O}_{1} \cap \tilde{O}_{2} \cap C / I\left(\tilde{O}_{2} \backslash \tilde{O}\right)=\tilde{O}_{1} \cap C / I\left(\tilde{O}_{2} \backslash \tilde{O}\right)$.

For Axioms $\mathrm{C}_{4} \mathrm{a}$ and $\mathrm{C}_{4} \mathrm{~b}$, consider any $\tilde{O}_{1}, \tilde{O}_{2}$ in $\mathcal{Q}(\mathcal{V} / I)$ such that $\tilde{O}_{1} \subseteq C / I\left(\tilde{O}_{2}\right)$, and consider any $\lambda$ in $\mathbb{R}_{>0}$ and $\tilde{u}$ in $\mathcal{V} / I$. Consider any option set $O_{2}$ in $\mathcal{Q}(\mathcal{V})$ such that $O_{2} / I=\tilde{O}_{2}$, let $O_{1}:=\left\{u \in O_{2}:[u] \in \tilde{O}_{1}\right\} \in \mathcal{Q}(\mathcal{V})$, and consider any $u$ in $\mathcal{V}$ such that $[u]=\tilde{u}$. We first prove that then $O_{1} \subseteq C\left(O_{2}\right)$. Indeed, consider any $v \in O_{1}$, meaning that $v \in O_{2}$ and $[v] \in \tilde{O}_{1}$, and therefore also $[v] \in C / I\left(\tilde{O}_{2}\right)$. Proposition 27 then guarantees that indeed $u \in C\left(O_{2}\right)$. We now infer from the coherence of $C\left[\right.$ Axioms $\mathrm{C}_{4} \mathrm{a}$ and $\left.\mathrm{C}_{4} \mathrm{~b}\right]$ that $\lambda O_{1} \subseteq C\left(\lambda O_{2}\right)$ and $O_{1}+\{u\} \subseteq C\left(O_{2}+\{u\}\right)$. Hence indeed $\lambda \tilde{O}_{1}=\lambda O_{1} / I=\left(\lambda O_{1}\right) / I \subseteq C / I\left(\left(\lambda O_{2}\right) / I\right)=$ $C / I\left(\lambda O_{2} / I\right)=C / I\left(\lambda \tilde{O}_{2}\right)$ and $\tilde{O}_{1}+\{\tilde{u}\}=O_{1} / I+\{[u]\}=\left(O_{1}+\{u\}\right) / I \subseteq C / I\left(\left(O_{2}+\{u\}\right) / I\right)=$ $C / I\left(O_{2} / I+\{\tilde{u}\}\right)=C / I\left(\tilde{O}_{2}+\{\tilde{u}\}\right)$, where the inclusions follow from Equation [14] and Proposition 27

For the converse implication, assume that $C / I$ is coherent. We show that $C$ satisfies Axioms $\mathrm{C}_{1} \mathrm{C}_{4} \mathrm{~b}$

For Axiom $\mathrm{C}_{1}$, consider any $O$ in $\mathcal{Q}(\mathcal{V})$ and assume ex absurdo that $C(O)=\varnothing$. Then it follows from Proposition 27 that $C / I(O / I)=C(O) / I=\varnothing / I=\varnothing$, which contradicts the coherence of $C / I$ [Axiom $\left.\mathrm{C}_{1}\right]$.

For Axiom $\mathrm{C}_{2}$, consider any $u, v$ in $\mathcal{V}$ such that $u<v$. Then $[u]<[v]$ by letting for example $w=0$ in Proposition 23. The coherence of $C / I$ [Axiom $\left.\mathrm{C}_{2}\right]$ then guarantees that $\{[v]\}=C / I(\{[u],[v]\})$, implying that $\{v\}=C(\{u, v\})$, by Proposition 27

For version (3) of Axiom $\mathrm{C}_{3}$ a consider any option sets $O, O_{1}, O_{2}$ in $\mathcal{Q}(\mathcal{V})$ such that $O_{1} \subseteq O_{2} \subseteq O$ and $O_{1} \cap C\left(O_{2}\right)=\varnothing$. Then Lemma 38 guarantees that $O_{1} / I \cap C / I\left(O_{2} / I\right)=\varnothing$. Because $O_{2} \subseteq O$, we have by Equation (14) that $O_{2} / I \subseteq O / I$, whence $O_{1} / I \cap C / I(O / I)=\varnothing$ by coherence of $C / I$ [version (3) of Axiom $\mathrm{C}_{3}$ a]. Using Lemma 38 again, we infer that indeed $O_{1} \cap C(O)=\varnothing$. 
For version (4) of Axiom $\mathrm{C}_{3} \mathrm{~b}$, consider any option sets $O, O_{1}, O_{2}$ in $\mathcal{Q}(\mathcal{V})$ such that $O \subseteq O_{1}$ and $O_{1} \cap C\left(O_{2}\right)=\varnothing$. We may assume without loss of generality that $O_{1} \subseteq O_{2}$ [if not, replace $O_{1}$ by $O_{1} \cap O_{2}$ and $O$ by $O \cap O_{2}$ ]. Let

$$
\bar{O}_{1}:=\left\{u \in O_{2}:[u] \in O_{1} / I\right\} \supseteq O_{1} \text { and } \check{O}:=\left\{u \in \bar{O}_{1}:[u] \cap O_{1} \subseteq O\right\},
$$

then $\bar{O}_{1} / I=O_{1} / I$. Because $C$ is compatible with $I$, we infer from Lemma 38 that the statements $O_{1} \cap C\left(O_{2}\right)=\varnothing, \bar{O}_{1} \cap C\left(O_{2}\right)=\varnothing$ and $\bar{O}_{1} / I \cap C / I\left(O_{2} / I\right)=\varnothing$ are equivalent, and therefore all hold. Observe that also $\check{O} \subseteq \bar{O}_{1} \subseteq O_{2}$, implying that $\check{O} / I \subseteq \bar{O}_{1} / I \subseteq O_{2} / I$ by Equation (14). That $\check{O} / I \subseteq \bar{O}_{1} / I$ and $\bar{O}_{1} / I \cap C / I\left(O_{2} / I\right)=\varnothing$ implies, together with the coherence of $C / I$ [version (4) of Axiom $\mathrm{C}_{3} \mathrm{~b}$ ], that $\bar{O}_{1} / I \cap C / I\left(O_{2} / I \backslash \breve{O} / I\right)=\varnothing$, and therefore also that $\left(\bar{O}_{1} / I \backslash \check{O} / I\right) \cap C / I\left(O_{2} / I \backslash \check{O} / I\right)=\varnothing$.

We now prove that $O_{2} / I \backslash \check{O} / I \subseteq\left(O_{2} \backslash O\right) / I$. Consider any $\tilde{u}$ in $O_{2} / I \backslash \check{O} / I$, and assume ex absurdo that $\tilde{u} \notin\left(O_{2} \backslash O\right) / I$, or in other words, that $\left(\forall w \in O_{2}\right)(w \notin O \Rightarrow \tilde{u} \neq[w])$, or equivalently, $\left(\forall w \in O_{2}\right)(\tilde{u}=[w] \Rightarrow w \in O)$. This implies that $\tilde{u} \cap O_{2} \subseteq O$, from which we infer on the one hand that $\tilde{u} \cap O_{1} \subseteq O$ since $O_{1} \subseteq O_{2}$ by assumption. On the other hand, we infer that $\left(\tilde{u} \cap O_{2}\right) / I \subseteq O / I$ by Equation (14), whence $\tilde{u} \in O / I$ because $\tilde{u} \in O_{2} / I$ by assumption, and therefore also $\tilde{u} \in O_{1} / I=\bar{O}_{1} / I$. Both observations together imply that $\tilde{u} \in \check{O} / I$, a contradiction with $\tilde{u} \in O_{2} / I \backslash \check{O} / I$. Coherence of $C / I$ [version (3) of Axiom $\mathrm{C}_{3}$ a with $\tilde{O}_{1}:=\bar{O}_{1} / I \backslash \check{O} / I$, $\tilde{O}_{2}:=O_{2} / I \backslash \check{O} / I$ and $\left.\tilde{O}:=\left(O_{2} \backslash O\right) / I\right]$ now implies that $\left(\bar{O}_{1} / I \backslash \tilde{O} / I\right) \cap C / I\left(\left(O_{2} \backslash O\right) / I\right)=\varnothing$.

We next prove that $\left(O_{1} \backslash O\right) / I \subseteq \bar{O}_{1} / I \backslash \check{O} / I$. Consider any $\tilde{u}$ in $\left(O_{1} \backslash O\right) / I$, so $\tilde{u}=[v]$ for some $v$ in $O_{1} \backslash O$. This already implies that $\tilde{u} \in \bar{O}_{1} / I$. Assume ex absurdo that $\tilde{u} \notin$ $\bar{O}_{1} / I \backslash \check{O} / I$, so $\tilde{u} \in \check{O} / I$, whence $\tilde{u} \cap O_{1} \subseteq O$. But we know that $v \in O_{1}$ and $v \in \tilde{u}$, so $v \in O$, a contradiction. We conclude form all this that $\left(\mathrm{O}_{1} \backslash O\right) / I \cap C / I\left(\left(\mathrm{O}_{2} \backslash O\right) / I\right)=\varnothing$, whence $\mathrm{O}_{1} \backslash \mathrm{O} \cap \mathrm{C}\left(\mathrm{O}_{2} \backslash \mathrm{O}\right)=\varnothing$ by Lemma 38, and because $C\left(\mathrm{O}_{2} \backslash \mathrm{O}\right) \subseteq \mathrm{O}_{2} \backslash \mathrm{O}$, indeed that $O_{1} \cap C\left(O_{2} \backslash O\right)=\varnothing$.

For Axioms $\mathrm{C}_{4} \mathrm{a}$ and $\mathrm{C}_{4} \mathrm{~b}$ consider any $O_{1}, O_{2}$ in $\mathcal{Q}(\mathcal{V})$ such that $O_{1} \subseteq C\left(O_{2}\right)$, and consider any $\lambda$ in $\mathbb{R}_{>0}$ and $\tilde{u}$ in $\mathcal{V}$. Then $O_{1} / I \subseteq C / I\left(O_{2} / I\right)$ by Equation (14) and Proposition 27, implying that $\left(\lambda O_{1}\right) / I=\lambda O_{1} / I \subseteq C / I\left(\lambda O_{2} / I\right)=C / I\left(\left(\lambda O_{2}\right) / I\right)$ and $\left(O_{1}+\{u\}\right) / I=$ $O_{1} / I+\{[u]\} \subseteq C / I\left(O_{2} / I+\{[u]\}\right)=C / I\left(\left(O_{2}+\{u\}\right) / I\right)$, where the inclusions follow from the coherence of $C / I$ [Axioms $\mathrm{C}_{4} \mathrm{a}$ and $\mathrm{C}_{4} \mathrm{~b}$ respectively]. This then implies that indeed $\lambda O_{1} \subseteq C\left(\lambda O_{2}\right)$ and $O_{1}+\{u\} \subseteq C\left(O_{2}+\{u\}\right)$.

Proof of Proposition 29. Let us denote $C:=\inf \left\{C_{i}: i \in \mathcal{I}\right\}$, then $C$ is a coherent choice function by Proposition 8 . We show that it is compatible with $I$. By assumption, and using Proposition 27, we have for all $i \in \mathcal{I}$ that

$$
C_{i}(O)=\left\{u \in O:[u] \in C_{i} / I(O / I)\right\} \text { for all } O \in \mathcal{Q}(\mathcal{V})
$$

Hence, for all $O \in \mathcal{Q}(\mathcal{V})$ :

$$
\begin{aligned}
C(O) & =\bigcup_{i \in \mathcal{I}} C_{i}(O)=\bigcup_{i \in \mathcal{I}}\left\{u \in O:[u] \in C_{i} / I(O / I)\right\} \\
& =\left\{u \in O:(\exists i \in \mathcal{I})[u] \in C_{i} / I(O / I)\right\} \\
& =\left\{u \in O:[u] \in \bigcup_{i \in \mathcal{I}} C_{i} / I(O / I)\right\} \\
& =\left\{u \in O:[u] \in\left(\inf \left\{C_{i} / I: i \in \mathcal{I}\right\}\right)(O / I)\right\},
\end{aligned}
$$

and the stated result now follows from Propositions 8 and 27 .

Lemma 40. Given $O_{1}$ and $O_{2}$ in $\mathcal{Q}$ such that $O_{1} \subseteq O_{2}$, we have that

$$
O_{1} \subseteq\left\{u \in O_{2}:[u] \in O_{1} / I\right\} .
$$


Proof. Consider any $v$ in $O_{1}$. Then $v \in O_{2}$ because $O_{1} \subseteq O_{2}$, and $[v] \in O_{1} / I$ by the definition of the quotient space, whence $v \in\left\{u \in O_{2}:[u] \in O_{1} / I\right\}$.

Proof of Proposition 30 . We first prove that $\approx_{C}$ is an equivalence relation. The reflexivity and symmetry are an immediate consequence of the definition (15). To prove transitivity, consider any $u, v, w$ in $\mathcal{V}$ and assume that $u \approx_{C} v$ and $v \approx_{C} w$. Consider any $O$ in $\mathcal{Q}$ and assume that $\{u, w\} \subseteq O$ and $u \in C(O)$. Then it suffices to prove that also $w \in C(O)$. Assume ex absurdo that $w \notin C(O)$, then we infer from Axiom $\mathrm{C}_{3}$ a that also $w \notin C(O \cup\{v\})$. Since $v \approx_{C} w$, we infer from Equation (15) that then $v \notin C(O \cup\{v\})$, and similarly, since $u \approx_{C} v$, that $u \notin C(O \cup\{v\})$, so $\{u, v\} \subseteq(O \cup\{v\}) \backslash C(O \cup\{v\})$. Axiom $\mathrm{C}_{3} \mathrm{~b}$ then tells us that $u \notin C(O)$, a contradiction.

To prove (ii), assume that $u \approx_{C} v$, and consider any $O \in \mathcal{Q}$ such that $\{u+w, v+w\} \subseteq O$ and $u+w \in C(O)$. Then it suffices to prove that also $v+w \in C(O)$. It follows from $u+w \in C(O)$ and Axiom $\mathrm{C}_{4} \mathrm{~b}$ that $u \in C(O-\{w\})$. Since $u \approx_{C} v$, we infer from Equation (15) that then also $v \in C(O-\{w\})$, whence, again by Axiom $\mathrm{C}_{4} \mathrm{~b}$ indeed $v+w \in C(O)$.

To prove (i), assume that $u \approx_{C} v$. We first prove that then also $-u \approx_{C}-v$. Indeed, by applying (ii) with $w:=-u-v$, we find that $-v \approx C-u$. Now use the symmetry of $\approx_{C}$. Next, consider any $O \in \mathcal{Q}$ such that $\{\lambda u, \lambda v\} \subseteq O$ and $\lambda u \in C(O)$. Then it suffices to prove that also $\lambda v \in C(O)$. The proof is trivial if $\lambda=0$. Because we have just proved that both $u \approx_{C} v$ and $-u \approx C-v$, we may now assume without loss of generality that $\lambda>0$. It follows from $\lambda u \in C(O)$ and Axiom $\mathrm{C}_{4} \mathrm{a}$ that $u \in C\left(\frac{1}{\lambda} O\right)$. Since $u \approx_{C} v$, we infer from Equation 15 that then also $v \in C\left(\frac{1}{\lambda} O\right)$, whence, again by Axiom $\mathrm{C}_{4} \mathrm{a}$, indeed $\lambda v \in C(O)$.

We complete the proof by showing that $I_{C}$ is a coherent set of indifferent options. To prove $I_{1}$, simply observe that $0 \approx_{C} 0$ by reflexivity of $\approx_{C}$. To prove $I_{2}$ it suffices to consider any $u \in \mathcal{V}_{>0}$, due to (i) It follows from Axiom $\mathrm{C}_{2}$ that both $0 \notin C(\{0, u\})$ and $u \in C(\{0, u\})$, so we infer from Equation (15) that $0 \psi_{C} u$, whence indeed $u \notin I_{C}$. To prove $I_{3}$, simply use (i) To prove $I_{4}$, simply use (ii) and the transitivity of $\approx_{C}$.

Proof of Proposition 31. For necessity, assume that $C$ is compatible with $I$, and hence

$$
(\forall O \in \mathcal{Q}) C(O)=\{u \in O:[u] \in C(O) / I\} .
$$

We need to prove that $I \subseteq I_{C}$, so consider any $u$ in $I$ and any $O \supseteq\{0, u\}$ in $\mathcal{Q}$ such that $u \in C(O)$ [There always is such an $O$, for instance $O:=\{0, u\}$, because $u \in I$ and therefore $[u] \in C / I(\{[u]\})=C / I(\{0, u\} / I)=C(\{0, u\}) / I$, by compatibility $]$. Then $[u] \in C(O) / I$ and because $[u]=[0]$, we find that $[0] \in C(O) / I$, so $0 \in C(O)$. Hence indeed $u \approx_{C} 0$, by Equation [15].

For sufficiency, assume that $I$ is a linear subspace of $I_{C}$, so $I \subseteq I_{C}$, then we need to prove that $C$ is compatible with $I$. In other words, if we consider any $O$ in $\mathcal{Q}$ and any $u \in O$ such that $[u] \in C(O) / I$, then we must prove that $u \in C(O)$. $[u] \in C(O) / I$ means that there is some $v \in C(O)$ such that $u-v \in I$, and therefore, by assumption, $u \approx C v$. Since $\{u, v\} \subseteq O$ and $v \in C(O)$, we infer from Equation (15) that indeed $u \in C(O)$.

Proof of Lemma 32 Fix $u$ and $v$ in $\mathcal{V}$.

For the direct implication, assume that the left-hand side holds. We first prove that this implies that both $u-v \notin D$ and $v-u \notin D$. Indeed, consider the left-hand side for the particular choice $O:=\{u, v\}$, leading to $\{0, v-u\} \cap D \neq \varnothing \Leftrightarrow\{0, u-v\} \cap D \neq \varnothing$, or equivalently, $v-u \in$ $D \Leftrightarrow u-v \in D$, because $0 \notin D$ by Axiom $\mathrm{D}_{1}$. So, if we had that $u-v \in D$ or $v-u \in D$, this would imply that both $u-v$ and $v-u$ would elements of $D$, and therefore also their sum $0=u-v+v-u \in D$ by Axiom $\mathbf{D}_{4}$ This contradicts Axiom $\mathbf{D}_{1}$ 
Next, consider any $w$ in $\mathcal{V}$ such that $w \notin\{u, v\}$, and consider the left-hand side for the particular choice $O:=\{u, v, w\}$. Then $O \supseteq\{u, v\}$ and therefore $O-\{u\} \cap D \neq \varnothing \Leftrightarrow$ $O-\{v\} \cap D \neq \varnothing$, which can rewritten as $\{0, v-u, w-u\} \cap D \neq \varnothing \Leftrightarrow\{u-v, 0, w-v\} \cap D \neq \varnothing$. But $0 \notin D$ by Axiom $\mathrm{D}_{1}$, whence $\{v-u, w-u\} \cap D \neq \varnothing \Leftrightarrow\{u-v, w-v\} \cap D \neq \varnothing$. But, since we have seen above that neither $u-v \in D$ nor $v-u \in D$, this can in turn be rewritten as $w-u \notin D \Leftrightarrow w-v \notin D$. This tells us that $w \in D+\{u\} \Leftrightarrow w \in D+\{v\}$ for all $w$ in $\mathcal{V}$, and therefore indeed $D+\{u\}=D+\{v\}$.

For the converse implication, assume that $D+\{u\}=D+\{v\}$. This immediately allows us to infer that $u-v \notin D$ and $v-u \notin D$. Consider any $O \supseteq\{u, v\}$. If $O=\{u, v\}$ then $O-\{u\} \cap D=\varnothing$ and $O-\{v\} \cap D=\varnothing$. Let $n:=|O|-2$ and assume therefore that $n \geq 1$. Label the elements of $O \backslash\{u, v\}$ as $w_{1}, \ldots, w_{n}$, without loss of generality. Then $O=\left\{u, v, w_{1}, \ldots, w_{n}\right\}$. Infer the following equivalences:

$$
\begin{aligned}
O-\{u\} \cap D \neq \varnothing & \Leftrightarrow\left\{0, v-u, w_{1}-u, \ldots, w_{n}-u\right\} \cap D \neq \varnothing \\
& \Leftrightarrow\left\{w_{1}-u, \ldots, w_{n}-u\right\} \cap D \neq \varnothing \\
& \Leftrightarrow(\exists i \in\{1, \ldots, n\}) w_{i}-u \in D \\
& \Leftrightarrow(\exists i \in\{1, \ldots, n\}) w_{i} \in D+\{u\}=D+\{v\} \\
& \Leftrightarrow(\exists i \in\{1, \ldots, n\}) w_{i}-v \in D \Leftrightarrow O-\{v\} \cap D \neq \varnothing,
\end{aligned}
$$

where the second equivalence follows from the fact that $0 \notin D$ [see Axiom $\mathrm{D}_{1}$ and our earlier observation that $v-u \notin D$.

Proof of Proposition 33 . We begin with the first statement. Consider any coherent choice function $C$ that is compatible with $I$. We must prove that $D_{C}+I \subseteq D_{C}$. Observe that for any $w$ in $\mathcal{V}$ :

$$
\begin{aligned}
w \in D_{C} & \Leftrightarrow 0 \notin C(\{0, w\}) \Leftrightarrow[0] \notin C / I(\{[0],[w]\}) \\
& \Leftrightarrow[w] \in D_{C / I},
\end{aligned}
$$

where the first equivalence follows from Proposition 12 and the second from the compatibility of $C$ with $I$. So, consider any $v$ in $D_{C}$ and any $u$ in $I$, then $[0] \notin C / I(\{[0],[v]\})$ and $[u]=[0]=I$, implying that $[v+u]=[v]+[u]=[v]+I=[v]+[0]=[v+0]=[v]$, and therefore $[0] \notin C / I(\{[0],[v+u]\})$, implying that indeed $v+u \in D_{C}$. The last statement follows directly from Equation (17).

We turn now towards the second statement. Consider any coherent set of indifferent options $I$ and any coherent set of desirable options $D$ such that $D+I \subseteq D$. We must prove that $C_{D}(O)=\left\{u \in O:[u] \in C_{D} / I(O / I)\right\}$ for all $O$ in $\mathcal{Q}(\mathcal{V})$. Due to Proposition 13 we know that $C_{D}(O)=\{u \in O:(\forall v \in O) v-u \notin D\}$ for all $O$ in $\mathcal{Q}(\mathcal{V})$, whence, by Proposition 24.

$$
\begin{aligned}
C_{D}(O) & =\{u \in O:(\forall v \in O)[v]-[u] \notin\{[w]: w \in D\}\} \\
& =\{u \in O:(\forall v \in O)[v]-[u] \notin D / I\} \\
& =\{u \in O:(\forall[v] \in O / I)[v]-[u] \notin D / I\} \\
& =\left\{u \in O:[u] \in C_{D / I}(O / I)\right\}
\end{aligned}
$$

for all $O$ in $\mathcal{Q}(\mathcal{V})$, because $C_{D / I}(O / I)=\{\tilde{u} \in O / I:(\forall \tilde{v} \in O / I) \tilde{v}-\tilde{u} \notin D / I\}$ for all $O / I$ in $\mathcal{Q}(\mathcal{V} / I)$. The fact that $C_{D} / I=C_{D / I}$ now follows from Proposition 27 .

Proof of Proposition 34. That the transformation $\operatorname{inv}_{\mathcal{P}}$ is linear, is immediate from its definition in Equation (16). 
To prove (i), observe that $\operatorname{inv}_{\mathcal{P}} \circ \pi^{t}=\frac{1}{|\mathcal{P}|} \sum_{\varpi \in \mathcal{P}} \varpi^{t} \circ \pi^{t}=\frac{1}{|\mathcal{P}|} \sum_{\varpi \in \mathcal{P}}(\pi \circ \varpi)^{t}=\operatorname{inv}_{\mathcal{P}}$, where the last equality holds because $\mathcal{P}$ is a group. For the second identity, observe that $\pi^{t} \circ \operatorname{inv}_{\mathcal{P}}=$ $\frac{1}{|\mathcal{P}|} \sum_{\varpi \in \mathcal{P}} \pi^{t} \circ \varpi^{t}=\frac{1}{|\mathcal{P}|} \sum_{\varpi \in \mathcal{P}}(\varpi \circ \pi)^{t}=$ inv $_{\mathcal{P}}$, where the first equality follows form the linear character of $\pi^{t}$ and the last equality holds because $\mathcal{P}$ is a group.

To prove (ii) observe that

$$
\operatorname{inv}_{\mathcal{P}} \circ \operatorname{inv}_{\mathcal{P}}=\frac{1}{|\mathcal{P}|} \sum_{\pi \in \mathcal{P}} \operatorname{inv}_{\mathcal{P}} \circ \pi^{t}=\frac{1}{|\mathcal{P}|} \sum_{\pi \in \mathcal{P}} \operatorname{inv}_{\mathcal{P}}=\operatorname{inv}_{\mathcal{P}}
$$

where the first equality is due to the linearity of $\operatorname{inv}_{\mathcal{P}}$ and the second due to (i)

To prove (iii) consider any $u$ in $\operatorname{kern}\left(\operatorname{inv}_{\mathcal{P}}\right)$. Then $\operatorname{inv}_{\mathcal{P}} u=0$ and therefore $u=u-$ $\operatorname{inv}_{\mathcal{P}} u=\frac{1}{|\mathcal{P}|} \sum_{\pi \in \mathcal{P}}\left(u-\pi^{t} u\right)$ is an element of $I_{\mathcal{P}}$. Conversely, consider any $u$ in $I_{\mathcal{P}}$, then $u=\sum_{k=1}^{n} \lambda_{k}\left(v_{k}-\pi_{k}^{t} v_{k}\right)$ for some $n$ in $\mathbb{N}, \lambda_{k}$ in $\mathbb{R}, v_{k}$ in $\mathcal{V}$ and $\pi_{k}$ in $\mathcal{P}$. But then inv $\mathcal{P} u=$ $\sum_{k=1}^{n} \lambda_{k}\left(\operatorname{inv}_{\mathcal{P}} v_{k}-\operatorname{inv}_{\mathcal{P}}\left(\pi_{k}^{t} v_{k}\right)\right)=0$, where the first equality is due to the linearity of inv $\mathcal{P}$ and the last due to (i). Hence indeed $u \in \operatorname{kern}\left(\operatorname{inv}_{\mathcal{P}}\right)$.

To prove (iv), consider any $u$ in $\operatorname{rng}\left(\operatorname{inv}_{\mathcal{P}}\right)$. Then $u=\operatorname{inv}_{\mathcal{P}} v$ for some $v$ in $\mathcal{V}$, and therefore $\pi^{t} u=\pi^{t}\left(\operatorname{inv}_{\mathcal{P}} v\right)=\left(\pi^{t} \circ \operatorname{inv}_{\mathcal{P}}\right) v=\operatorname{inv}_{\mathcal{P}} v=u$ for all $\pi$ in $\mathcal{P}$, where the third equality follows from (i) Hence indeed $u \in \mathcal{V}_{\mathcal{P}}$. Conversely, consider any $u$ in $\mathcal{V}_{\mathcal{P}}$. Then $\pi^{t} u=u$ for all $\pi$ in $\mathcal{P}$, and therefore $u=\operatorname{inv}_{\mathcal{P}} u$, whence indeed $u \in \operatorname{rng}\left(\operatorname{inv}_{\mathcal{P}}\right)$.

For the last statement, simply observe that $v \in u / I_{\mathcal{P}} \Leftrightarrow v-u \in I_{\mathcal{P}} \Leftrightarrow \operatorname{inv} \mathcal{P}(v-u)=0 \Leftrightarrow$ $\operatorname{inv}_{\mathcal{P}} v=\operatorname{inv}_{\mathcal{P}} u$, where the second equivalence follows from (iii) and the last from the linearity of $\operatorname{inv}_{\mathcal{P}}$.

\section{REFERENCES}

[1] Mark A. Aizerman. New problems in the general choice theory. Social Choice and Welfare, 2:235-282, 1985. doi: 10.1007/BF00292690.

[2] Francis J. Anscombe and Robert J. Aumann. A definition of subjective probability. The Annals of Mathematical Statistics, 34:199-205, 1963. doi: 10.1214/aoms/1177704255. URL http://www.jstor.org/stable/2991295.

[3] Raveendra N. Batra and Prasanta K. Pattanaik. On some suggestions for having non-binary social choice functions. Theory and Decision, 3:1-11, 1972. doi: 10.1007/ BF00139349.

[4] Seamus Bradley. How to choose among choice functions. In Thomas Augustin, Serena Doria, Enrique Miranda, and Erik Quaeghebeur, editors, ISIPTA '15: Proceedings of the Ninth International Symposium on Imprecise Probability: Theories and Applications, pages 57-66, Pescara, 2015. Aracne.

[5] Inés Couso and Serafín Moral. Sets of desirable gambles: conditioning, representation, and precise probabilities. International Journal of Approximate Reasoning, 52(7): 1034-1055, 2011. doi: 10.1016/j.ijar.2011.04.004.

[6] Brian A. Davey and Hilary A. Priestley. Introduction to Lattices and Order. Cambridge University Press, Cambridge, 1990. doi: 10.1017/CBO9780511809088.

[7] Jasper De Bock and Gert de Cooman. Credal networks under epistemic irrelevance: the sets of desirable gambles approach. International Journal of Approximate Reasoning, 56(B):178-207, 2015. ISSN 0888-613X. doi: 10.1016/j.ijar.2014.07.002.

[8] Jasper De Bock, Arthur Van Camp, Márcio Alves Diniz, and Gert de Cooman. Representation theorems for partially exchangeable random variables. Fuzzy Sets and Systems, 284:1-30, 2016. doi: 10.1016/j.fss.2014.10.027.

[9] Gert de Cooman and Enrique Miranda. Symmetry of models versus models of symmetry. In W. L. Harper and G. R. Wheeler, editors, Probability and Inference: 
Essays in Honor of Henry E. Kyburg, Jr., pages 67-149. King's College Publications, 2007.

[10] Gert de Cooman and Enrique Miranda. Irrelevance and independence for sets of desirable gambles. Journal of Artificial Intelligence Research, 45:601-640, 2012. doi: 10.1613/jair.3770.

[11] Gert de Cooman and Erik Quaeghebeur. Exchangeability and sets of desirable gambles. International Journal of Approximate Reasoning, 53(3):363-395, 2012. doi: 10.1016/ j.ijar.2010.12.002. Precisely imprecise: A collection of papers dedicated to Henry E. Kyburg, Jr.

[12] Gert de Cooman and Matthias C. M. Troffaes. Dynamic programming for deterministic discrete-time systems with uncertain gain. International Journal of Approximate Reasoning, 39:257-278, 2005. doi: 10.1016/j.ijar.2004.10.004.

[13] Gert de Cooman, Jasper De Bock, and Márcio Alves Diniz. Coherent predictive inference under exchangeability with imprecise probabilities. Journal of Artificial Intelligence Research, 52:1-95, 2015. doi: 10.1613/jair.4490.

[14] Peter C. Fishburn. Should social choice be based on binary comparisons? Journal of Mathematical Sociology, 1:133-142, 1971. doi: 10.1080/0022250X.1971.9989792.

[15] Junnan He. A generalized unification theorem for choice theoretic foundations: Avoiding the necessity of pairs and triplets. Economics Discussion Paper 2012-23, Kiel Institute for the World Economy, 2012. URL http://www.economics-ejournal. org/economics/discussionpapers/2012-23.

[16] Joseph B. Kadane, Mark J. Schervish, and Teddy Seidenfeld. A Rubinesque theory of decision. Institute of Mathematical Statistics Lecture Notes-Monograph Series, 45:4555, 2004. doi: 10.1214/lnms/1196285378. URL http://www.jstor.org/stable/4356297.

[17] Isaac Levi. The Enterprise of Knowledge. MIT Press, London, 1980.

[18] Serafín Moral. Epistemic irrelevance on sets of desirable gambles. Annals of Mathematics and Artificial Intelligence, 45(1-2):197-214, 2005. doi: 10.1007/ s10472-005-9011-0.

[19] Erik Quaeghebeur. Desirability. In Thomas Augustin, Frank P. A. Coolen, Gert de Cooman, and Matthias C. M. Troffaes, editors, Introduction to Imprecise Probabilities, chapter 1, pages 1-27. John Wiley \& Sons, 2014. doi: 10.1002/9781118763117.ch1.

[20] Erik Quaeghebeur, Gert de Cooman, and Filip Hermans. Accept \& reject statementbased uncertainty models. International Journal of Approximate Reasoning, 57: 69-102, 2015. doi: 10.1016/j.ijar.2014.12.003. URL http://arxiv.org/abs/1208.4462.

[21] Herman Rubin. A weak system of axioms for "rational" behavior and the nonseparability of utility from prior. Statistics \& Risk Modeling, 5(1-2):47-58, 1987. doi: 10.1524/strm.1987.5.12.47.

[22] Thomas Schwartz. On the possibility of rational policy evaluation. Theory and Decision, 1:89-106, 1970. doi: 10.1007/BF00132454.

[23] Thomas Schwartz. Rationality and the myth of the maximum. Noûs, 6(2):97-117, 1972. doi: $10.2307 / 2216143$.

[24] Teddy Seidenfeld. Decision theory without "independence" or without "ordering". Economics and Philosophy, 4:267-290, October 1988. doi: 10.1017/S0266267100001085.

[25] Teddy Seidenfeld, Mark J. Schervish, and Joseph B. Kadane. A representation of partially ordered preferences. The Annals of Statistics, 23:2168-2217, 1995. doi: 10.1214/aos/1034713653. Reprinted in [26], pp. 69-129.

[26] Teddy Seidenfeld, Mark J. Schervish, and Joseph B. Kadane. Rethinking the Foundations of Statistics. Cambridge University Press, Cambridge, 1999. 
[27] Teddy Seidenfeld, Mark J. Schervish, and Joseph B. Kadane. Coherent choice functions under uncertainty. Synthese, 172(1):157-176, 2010. doi: 10.1007/ s11229-009-9470-7.

[28] Amartya Sen. Choice functions and revealed preference. The Review of Economic Studies, 38(3):307-317, July 1971. doi: 10.2307/2296384.

[29] Amartya Sen. Social choice theory: A re-examination. Econometrica, 45:53-89, 1977. doi: $10.2307 / 1913287$.

[30] Matthias C. M. Troffaes. Decision making under uncertainty using imprecise probabilities. International Journal of Approximate Reasoning, 45(1):17-29, 2007. doi: 10.1016/j.ijar.2006.06.001.

[31] Arthur Van Camp, Gert de Cooman, Enrique Miranda, and Erik Quaeghebeur. Modelling indifference with choice functions. In Thomas Augustin, Serena Doria, Enrique Miranda, and Erik Quaeghebeur, editors, ISIPTA '15: Proceedings of the Ninth International Symposium on Imprecise Probability: Theories and Applications, pages 305-314, Pescara, 2015. Aracne.

[32] Arthur Van Camp, Enrique Miranda, and Gert de Cooman. Lexicographic choice functions without Archimedeanicity. In M.B. Ferraro, P. Giordani, B. Vantaggi, M. Gagolewski, O. Hryniewicz, M.A. Gil, and P. Grzegorzewski, editors, Soft Methods for Data Science, pages 479-486. Springer, 2016.

[33] John von Neumann and Oskar Morgenstern. Theory of Games and Economic Behaviour. Princeton University Press, 3rd edition, 1972. ISBN 0691041830.

[34] Peter Walley. Statistical Reasoning with Imprecise Probabilities. Chapman and Hall, London, 1991.

[35] Peter Walley. Towards a unified theory of imprecise probability. International Journal of Approximate Reasoning, 24(2-3):125-148, 2000. doi: 10.1016/S0888-613X(00) 00031-1.

[36] Marco Zaffalon and Enrique Miranda. Desirability and the birth of incomplete preferences. ArXiv e-prints: arXiv:1506.00529, June 2015.

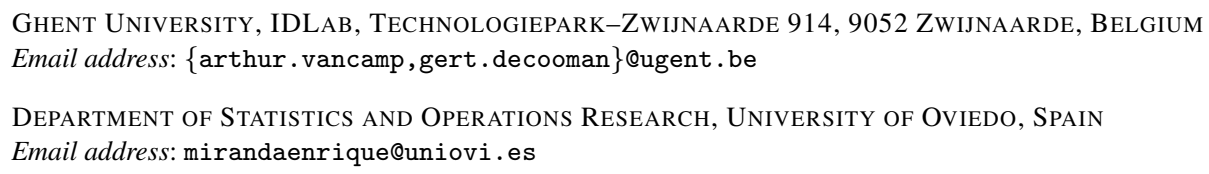

